\title{
Unique Reactivity of Fluorinated Molecules with Transition Metals
}

\author{
Silvia Catalán ${ }^{\mathrm{ab}}$, Sócrates B. Munoz ${ }^{\mathrm{c}}$, and Santos Fustero*ab
}

\begin{abstract}
Organofluorine and organometallic chemistry by themselves constitute two potent areas in organic synthesis. Thus, the combination of both offers many chemical possibilities and represents a powerful tool for the design and development of new synthetic methodologies leading to diverse molecular structures in an efficient manner. Given the importance of the selective introduction of fluorine atoms into organic molecules and the effectiveness of transition metals in $\mathrm{C}-\mathrm{C}$ and $\mathrm{C}$-heteroatom bond formation, this review represents an interesting read for this aim.
\end{abstract}

Keywords: Catalysis · Copper · Cross coupling · Fluorine $\cdot$ Gold $\cdot$ Metathesis $\cdot$ Pauson-Khand reaction · Silver · Transition metal

\section{Introduction}

The use of transition metals in organic synthesis has been immense throughout history and it is experiencing continuous development and increasing application. Over the past decades, transition metalcatalyzed/mediated reactions have become one of the most efficient methods for $\mathrm{C}-\mathrm{C}$ and $\mathrm{C}$-heteroatom bond formation, in particular for the construction of heterocyclic molecules. Indeed, the most important organic reactions, such as Suzuki, Sonogashira, Heck, olefin and alkyne metathesis and so forth, are catalyzed by transition metal complexes. Owing to the ongoing innovation in this area, transition metals have been successfully incorporated in the ever-growing field of organofluorine chemistry with remarkable and often unexpected results.

It is well-known that the selective introduction of fluorine atoms into organic molecules has become one of the most efficient methods for modulation of their biological properties ${ }^{[1]}$ and a common strategy in new drug discovery processes, [2] either by modifying known natural products or by searching for more active

\footnotetext{
${ }^{*}$ Correspondence: Prof. S. Fustero ${ }^{\text {ab }}$

Tel.: +3496354 4279

E-mail: santos.fustero@uv.es

aDepartamento de Química Orgánica

Universidad de Valencia

E-46100 Burjassot, Spain

'Laboratorio de Moléculas Orgánicas

Centro de Investigación Príncipe Felipe

E-46012, Valencia, Spain

'Loker Hydrocarbon Research Institute

Department of Chemistry

University of Southern California, Los Angeles

CA 90089, USA
}

drug-like molecules. In this way, the presence of fluorine in drugs has an extraordinary impact on a variety of medical applications, including anti-inflammatory, antibacterial, antitumor and antiviral agents, CNS-modulator compounds, or drugs for lowering blood cholesterol levels such as atorvastatin (Lipitor ${ }^{\circledR}$ ), developed by Pfizer and one of the best selling drugs in the world. Furthermore, the use of ${ }^{18} \mathrm{~F}$-radiolabelled compounds employed in Positron Emission Tomography (PET) experiments has exponentially grown in the last few years. Therefore, the important role of the fluorine atom in medicinal chemistry is obvious, ${ }^{[1 b, 3]}$ and it can be affirmed that fluorine chemistry will contribute significantly to the future progress in medicine. ${ }^{[4]}$

In this review, we provide an overview of recent advances in the field of fluorine chemistry combined with transition metal catalysis that have been reported in the last five years (Fig. 1). Given the significant number of reviews, which have

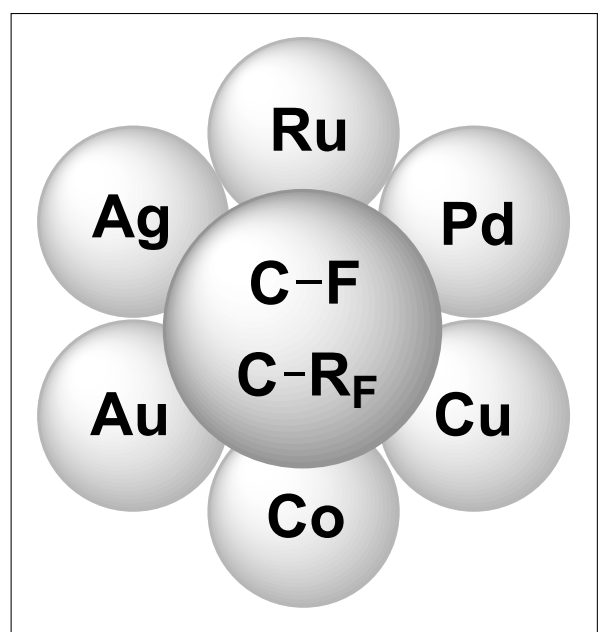

Fig. 1. Fluorine and transition metals. appeared over the last few years about transition metal-mediated fluorination, difluoromethylation, and trifluoromethylation methods, ${ }^{[1 \mathrm{~d}, 5]}$ we have also sought to compile some examples which display a significantly different reactivity of fluorinated molecules than their non-fluorinated analogs.

\section{Transition Metal-mediated Reactions}

\subsection{Cobalt-mediated Pauson- Khand Reaction}

The Pauson-Khand reaction (PKR) is one of the key methods for the construction of cyclopentenone derivatives, which can in turn undergo diverse chemical transformations. ${ }^{[6]}$ Nevertheless, the control of the regiochemical outcome of the PKR is an important setback. Whereas the intermolecular PKR of terminal alkynes is completely regioselective, always providing $\alpha$-substituted cyclopentenones, the less frequently used internal nonsymmetric alkynes can afford two regioisomers. That is not the case for fluorinated alkynes, in which the electron-withdrawing effect of the fluorinated substituents determines the regioselectivity (Scheme 1).

In contrast to the intramolecular version, no report on intermolecular PKR of fluorinated alkynes had been described until Riera, Fustero and coworkers reported the first example with norbornadiene in the presence of a stoichiometric amount of dicobalt hexacarbonyl complex $\left[\mathrm{Co}_{2}(\mathrm{CO})_{8}\right]$ under standard thermal conditions (Scheme 2). ${ }^{[7]}$

Surprisingly, in all examples, the fluorinated moiety was $\alpha$ to the carbonyl group, as opposed to the standard selectivity. The authors suggested that the regioselectivity 


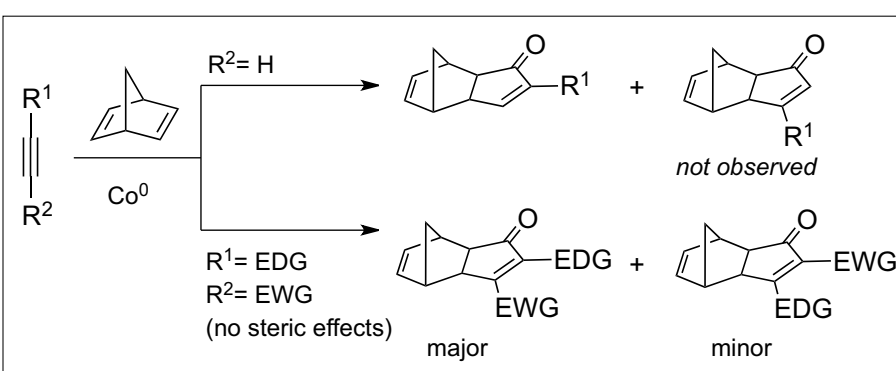

$E D G=$ electron-donating group $E W G=$ electron-withdrawing group

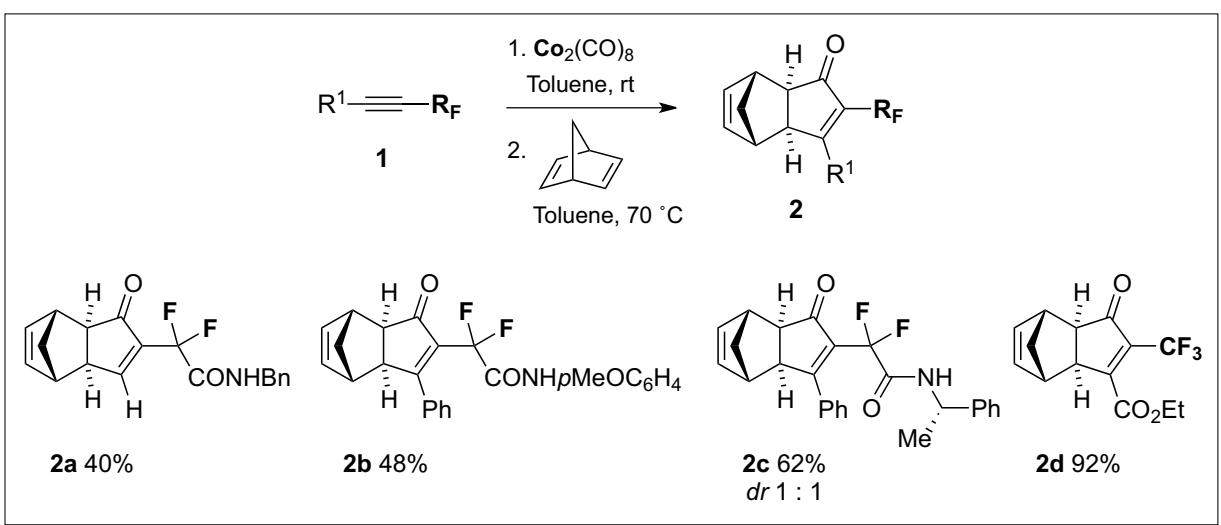

Scheme 2. Intermolecular PKR of fluorinated alkynes.

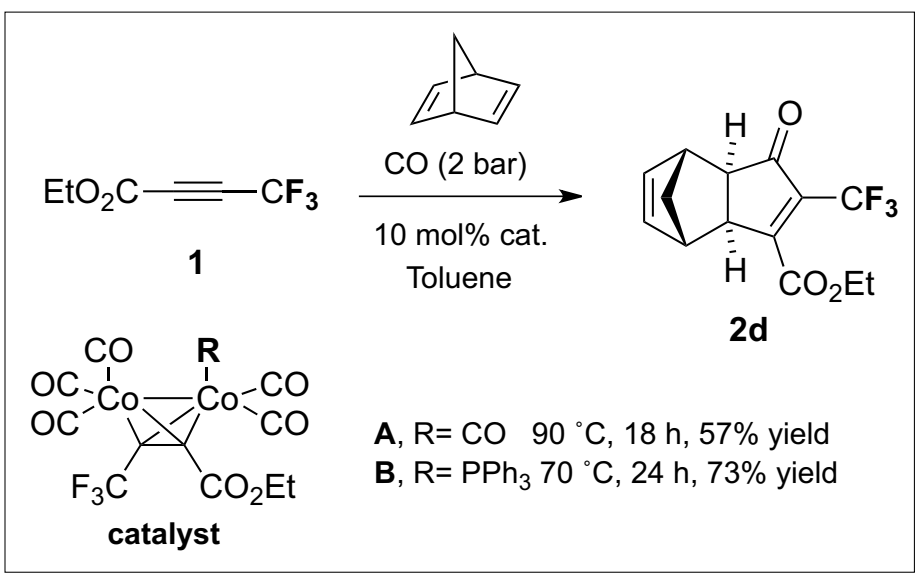

Scheme 3. Catalytic PKR of 1 with norbornadiene.

cheme 1. General trends of the regioselectivity of intermolecular PKR. served, bearing a $\mathrm{CF}_{3}$ group at the $\alpha$ position to the enone. ${ }^{[8]}$

Furthermore, this methodology was extended to the removal of the trifluoromethyl group by treatment with DBU in nitromethane/water, affording the unexpected $\beta$-substituted PK adducts (Scheme $5)$. The mechanism proposed consisted of a Michael addition of nitromethane which would entail a loss of fluoride giving gemdifluoroenone $\mathbf{3}$. Then, a conjugative addition of water would take place, followed by a retro-aldol reaction, which would afford intermediate 4. The final cyclopentenone 5 would be formed by retro-Michael reaction on enol 4 (Scheme 5). This method constituted a novel procedure to obtain PK substrates from terminal alkynes with inverse regiochemistry employing the trifluoromethyl group as a steering group.

In contrast, Konno et al. reported the synthesis of 2-fluoralkyl-2-cyclopentenones via Co-mediated PKR of several trifluoromethyl alkynes with 2-norbornene instead of norbornadiene (Scheme 6, part A). [9]

In this process, the regioselectivity was not complete and the PK adducts were obtained as isomeric mixtures, despite applying the same strategy but different reaction conditions. Additionally, the influence of alkyne substitution was studied, supporting the idea that the steric hindrance is a determining factor in the regiochemical outcome of the reaction (Scheme 6, part B). Recently, Riera and coworkers have confirmed these results by expanding their previous work to norbornene and also ethylene (Scheme 7). ${ }^{[10]}$ As Konno et al., they obtained mixtures of regioisomers with norbornene under established reaction conditions, identifying $\alpha$-fluorinated cyclopentenone as the major isomer, whereas

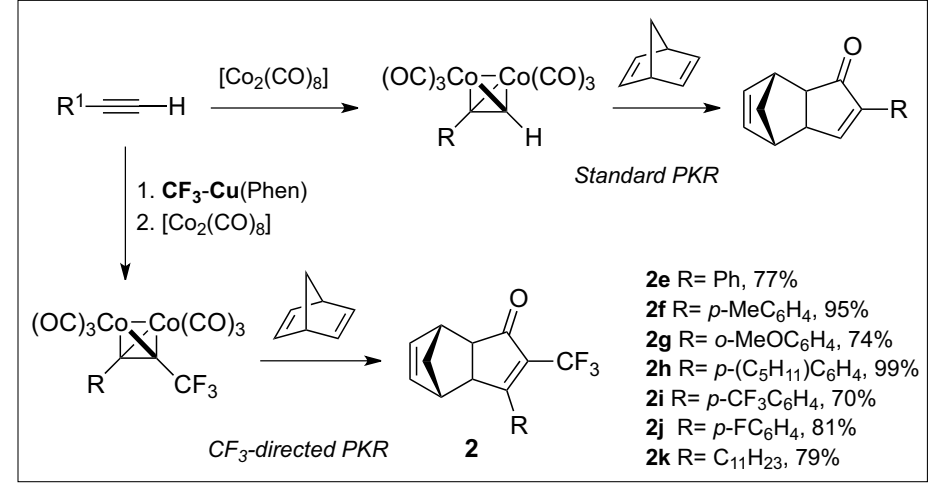

Scheme 4. Standard intermolecular PKR for internal alkynes and the sequence for internal $\mathrm{CF}_{3}$-substituted alkynes.

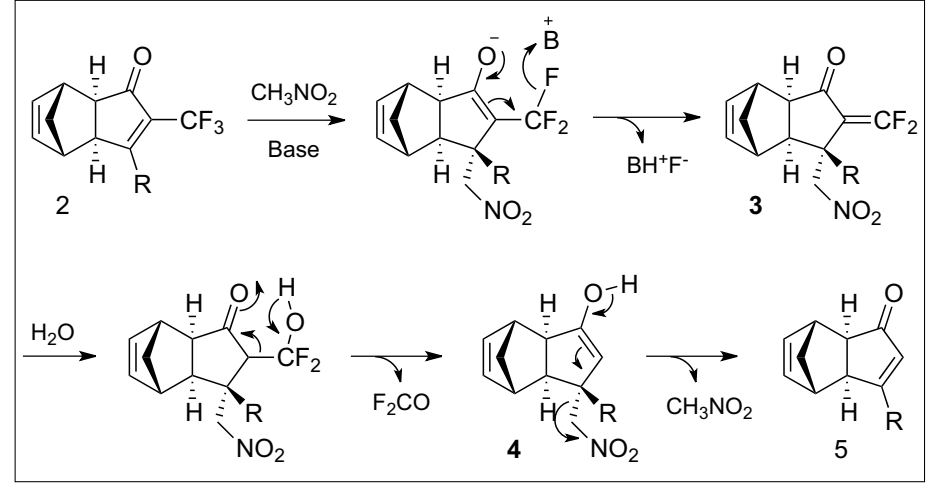

Scheme 5. Postulated mechanism for the $\mathrm{CF}_{3}$ elimination. of PK adducts was not determined by electronic but by steric effects mainly. In addition, a catalytic version of this process was also developed successfully by Riera and coworkers, using $10 \mathrm{~mol} \%$ of the cobaltalkyne complex formed in situ (Scheme 3).
Subsequently, the scope of this process was studied employing a wide variety of internal $\mathrm{CF}_{3}$-substituted alkynes containing aromatic, aliphatic, and olefinic substituents (Scheme 4). In all cases, the formation of a single regioisomer was ob- the minor one was the regioisomer with the $\mathrm{CF}_{3}$ at the $\beta$-position. Nevertheless, only one PK adduct, $\alpha$-trifluoromethyl cyclopentenone, was observed when using ethylene as the alkene, although the chemical yields were moderate. 


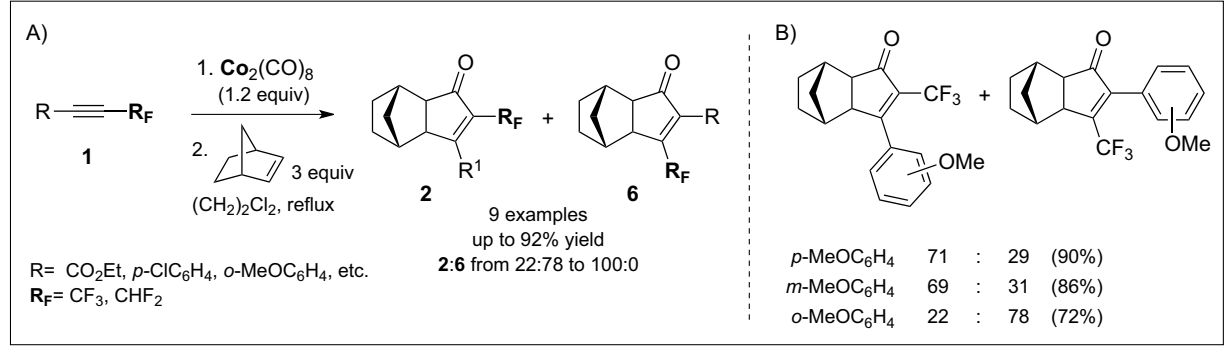

Scheme 6. PKR of $\mathrm{CF}_{3}$-substituted alkynes 1 with 2-norbornene and influence of alkyne substitution in the regiochemical outcome.

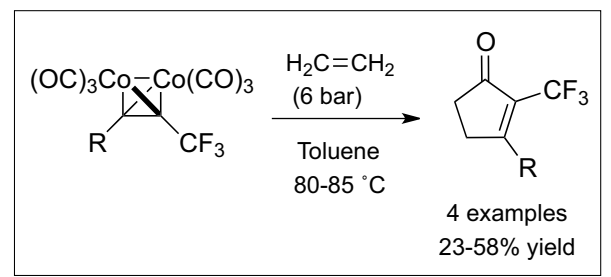

Scheme 7. Intermolecular PKR of 1 with ethylene.
Likewise, only a few examples of the intramolecular PKR of fluorinated enynes have been reported thus far. In this context, Ichikawa and Nadano described an attractive route to synthesize steroid and alkaloid analogs containing fluorine groupings in their structures. ${ }^{[11]}$ In particular, pyrrolidine ring-fused fluorinated cyclopentenone derivatives $\mathbf{1 1}$ were synthesized through a

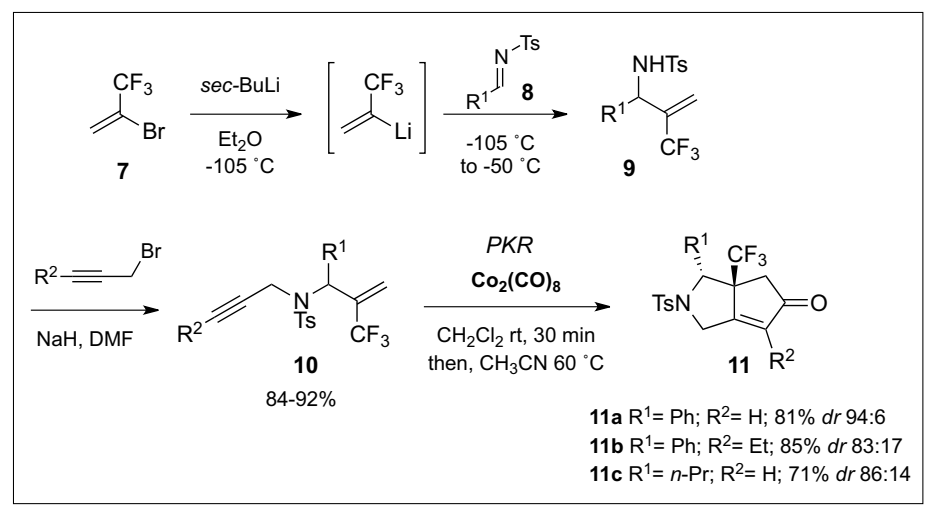
mediated intramolecular PKR in the synthesis of 11.
Scheme 8. Co-

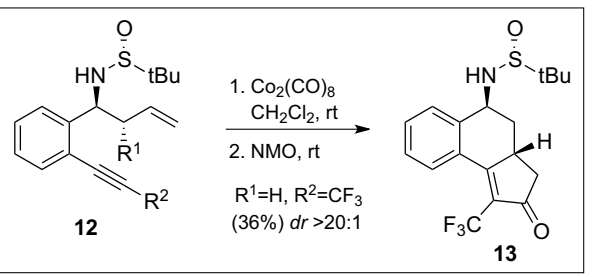

Scheme 9. Intramolecular PKR in the synthesis of tricyclic amines 13.
Co-mediated intramolecular PKR from $N$-propargyl- $N$-[2-(trifluoromethyl)allyl] a sequence of (trifluoromethyl)vinylation of imines $\mathbf{8}$, followed by propargylation of amides 9 (Scheme 8).

More recently, Fustero et al. described, for the first time, an intramolecular PKR amides 10, which were in turn prepared by

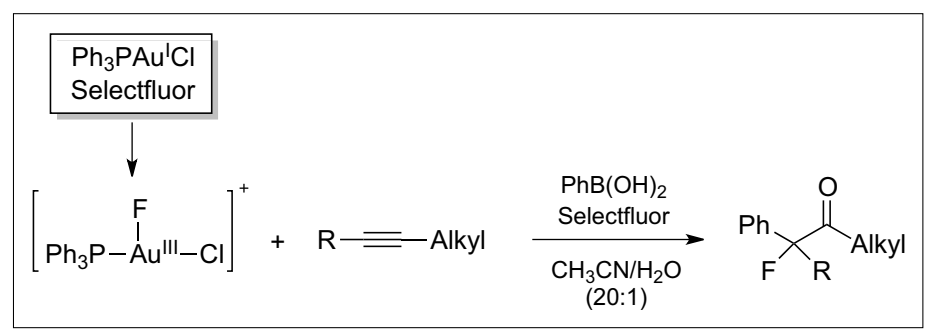

Scheme 11. Au(III)-catalyzed cross-coupling with arylboronic acids.
Scheme 10. Goldcatalyzed one-pot cascade reaction.

of a $\mathrm{CF}_{3}$-alkyne tethered to a chiral arylhomoallylic sulfonamide skeleton in the asymmetric synthesis of tricyclic amines 13 (Scheme 9). ${ }^{[12]}$ Building on the principles of diversity-oriented synthesis, different aryl, alkyl or terminal alkyne derivatives were subjected to this protocol with $\mathrm{CF}_{3}$-alkyne being of particular interest. Although the isolated yield of the resulting $\mathrm{CF}_{3}-\mathrm{PK}$ adduct was moderate $(36 \%$ yield), diastereoselectivity was excellent $(d r>20: 1)$.

\subsection{Gold-catalyzed Reactions}

Arguably, gold chemistry attracts sufficient attention by itself owing to the exceptional properties of gold complexes to activate multiple bonds such as alkenes, alkynes, allenes or enynes, and promote surprising transformations in a single synthetic operation. By adding the unique reactivity of fluorinated substrates to this, an $\mathrm{Au}-\mathrm{F}$ bond results in a great combination which offers many chemical possibilities.

In the last few years, important advances in this chemistry have been made and many reports have appeared in the literature involving the study of the influence of fluorine in gold-catalyzed processes. Based upon the pioneering studies reported by Zhang and others ${ }^{[13]}$ about the evidence for the existence of an $\mathrm{Au}(\mathrm{I}) / \mathrm{Au}(\mathrm{III})$ catalytic cycle, Hammond and coworkers developed a direct route to $\alpha$-substituted $\alpha$-fluoroketones from alkynes by a goldcatalyzed one-pot cascade reaction (Scheme 10). ${ }^{[14]}$ Only the combination of the pre-catalyst $\mathrm{Ph}_{3} \mathrm{PAuCl}$ and Selectfluor as an external oxidant promoted the formation of the catalytically active gold(III) complex, which was able to trigger a hydration/oxidative arylation/electrophilic fluorination sequence under mild conditions in good yields and moderate regioselectivities.

Thus, Hammond proposed that Selectfluor was in fact the activator of the cationic gold complex. This hypothesis of an oxidation of gold(I) to gold(III) by ' $\mathrm{F}^{+}$' was also supported by the studies of Toste and coworkers. ${ }^{[15]}$ They isolated, for first time, a gold(III) fluoride complex as a dimer which was successfully characterized by X-ray crystallography. The gold(III) fluoride species showed a unique reactivity in cross-coupling reactions with arylboronic acids (Scheme 11).

In this context, a tandem gold-catalyzed process starting from propargyl acetates was reported by Nevado and de Haro.[16] Selectfluor was used again as an oxidant for the synthesis of $\alpha$-fluoroenones 14 through a tandem [3,3]-sigmatropic rearrangement-fluorination process (Scheme 12). In contrast to previous statements, the cationic gold(I) complex was the catalytically active species which promoted a 1,3-migration of the acyloxy moiety by activation of the alkyne. The resulting vinyl$\mathrm{Au}(\mathrm{I})$ intermediate $\mathbf{A}$ was then oxidized to gold(III) (B) by Selectfluor. Reductive elimination was considered the last step to transform the $\mathrm{C} s p^{2}-\mathrm{Au}(\mathrm{III})$ bond into the $\mathrm{Cs} p^{2}-\mathrm{F}$ bond, giving rise to $\alpha$-fluoroenone derivatives $\mathbf{1 4}$ in good yields.

Nevertheless, Gouverneur and coworkers reported a similar methodology 


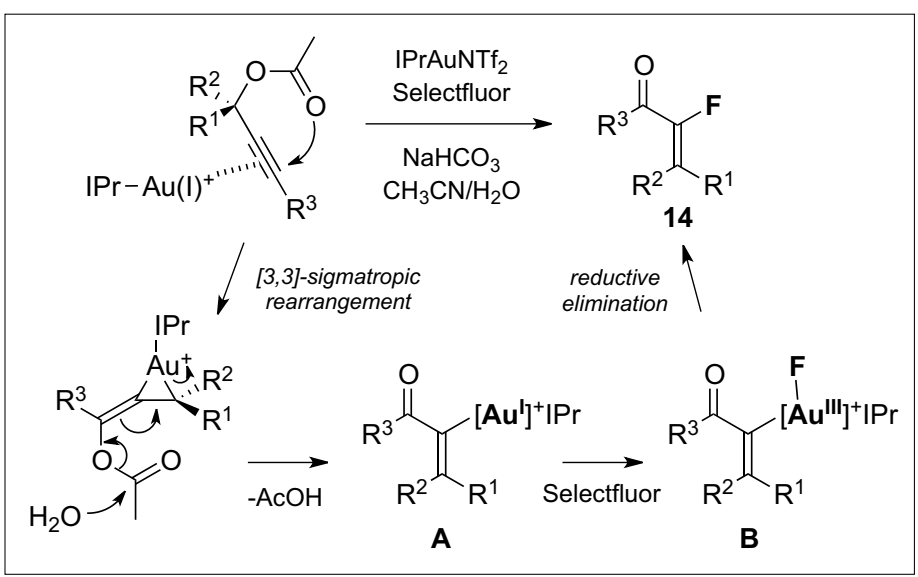

Scheme 12. Tandem gold-catalyzed [3,3]-sigmatropic rearrangement-fluorination process to obtain $\alpha$-fluoroenones. ${ }^{[17]}$ In this case, the proposed mechanism did not involve a gold-catalyzed electrophilic fluorination step but gold promoted the isomerization of the propargylic ester to give allenyl acetate, which then reacted with Selectfluor (Scheme 13).

Based upon these observations, Hammond and coworkers employed allenyl carbinol esters $\mathbf{1 5}$ in the stereoselective synthesis of fluoroalkyl $\alpha, \beta$-unsaturated ketones 17 (Scheme 14). ${ }^{[18]}$ First, isomerization of the allene substrate in the presence of gold produced a diene intermediate (16), which quickly reacted with Selectfluor to exclusively give the $E$ isomer of the corresponding fluorinated ketone $\mathbf{1 7}$ in high yield.

More recently, a gold-catalyzed tandem aminocyclization/electrophilic fluorination sequence has been reported by Arcadi et al. for the synthesis of 3,3-difluoroand 3-fluoroindole derivatives 19 and 20 (Scheme 15).[19] Starting from $o$-alkynyl anilines (18) and by using an excess of Selectfluor in EtOH at room temperature, necessarily in the presence of a gold(I) or gold(III) catalyst (e.g. $\mathrm{Ph}_{3} \mathrm{PAuNTf}_{2}, \mathrm{AuCl}$, $\mathrm{AuCl}_{3}$ or $\left.\mathrm{NaAuCl} \cdot 2 \mathrm{H}_{2} \mathrm{O}\right)$, an assorted library of $\mathrm{C}(3)$ difluorinated indoles 19 was prepared in good yields (Scheme 15).

By slightly modifying the reaction conditions, the selective formation of $\mathrm{C}(3)$ monofluorinated derivatives $\mathbf{2 0}$ was feasible (Scheme 15 and 16). Considering that Selectfluor is able to oxidize gold(I) to gold(III) and the latter also catalyzed the cyclization/fluorination process, two possible mechanism pathways were proposed: the one (a) involving an $\mathrm{Au}(\mathrm{I}) / \mathrm{Au}$ (III) catalytic cycle and a second (b) involving a protodeauration followed by a non-catalyzed fluorination step on the indole intermediate (Scheme 16).

On the other hand, one of the most commonly used methods to generate fluorinated compounds consists of using fluorinated building blocks for assembling the new molecule. The presence of fluorine in these small fragments confers a particular reactivity upon them. Therefore, transi-

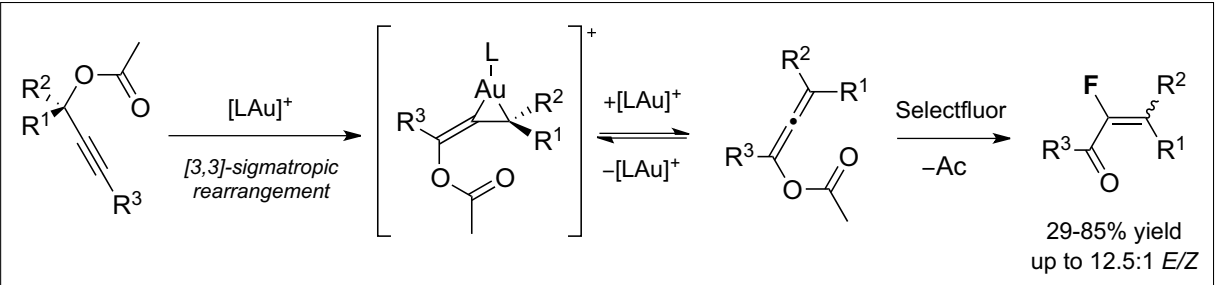

Scheme 13. Alternative proposal for the formation of $\alpha$-fluoroenones catalyzed by gold.
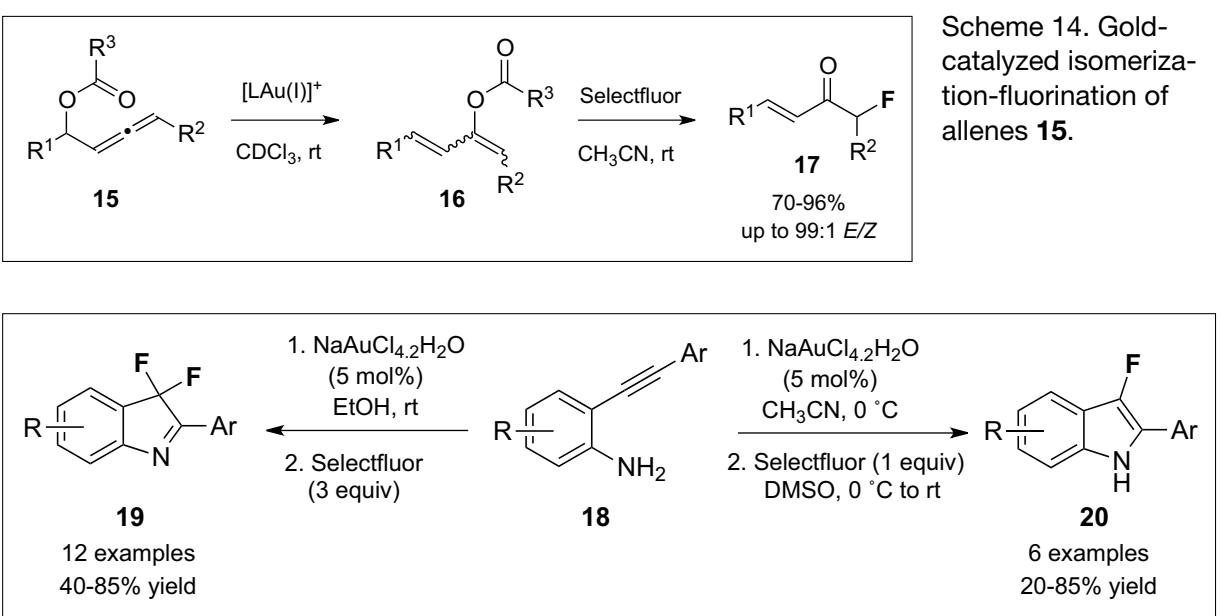

Scheme 15. Gold-catalyzed tandem aminocyclization/electrophilic fluorination of 18

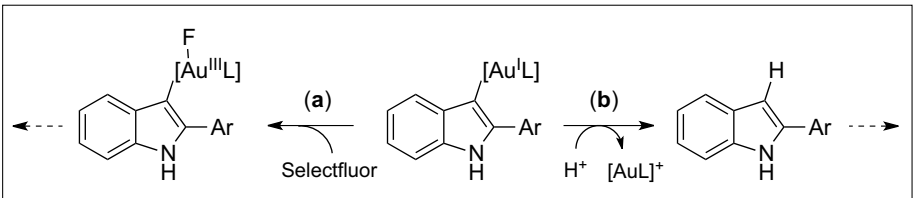

Scheme 16

Proposed mechanism pathways for Aucatalyzed cyclizationfluorination process.

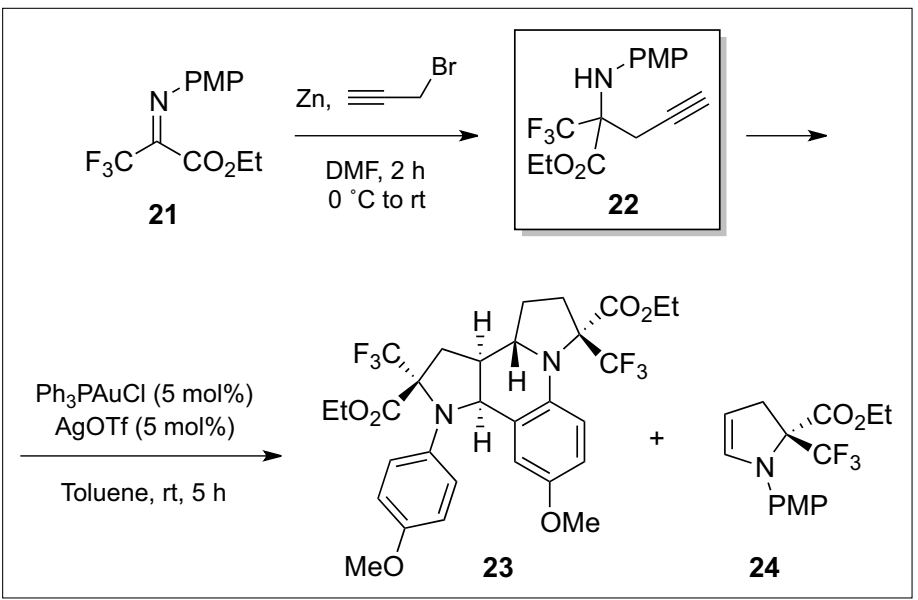

Scheme 17. Goldcatalyzed stereoselective tandem hydroamination-formal aza-Diels-Alder reaction of fluorinated $\alpha$-substituted propargylic amino esters 22. 
of gold salts. Unexpectedly, the isolated product was identified as a tetracycle $\mathbf{2 3}$ whose formation involved four new bonds and five stereocenters, as a single diastereoisomer. A gold(I)-catalyzed intramolecular hydroamination followed by a formal aza-Diels-Alder reaction was the sequence proposed for the final outcome, which was further extended to non-fluorinated substrates.

Moreover, when the aromatic group attached to the nitrogen atom was less activated, the process evolved to the hydroamination product, fluorinated pyrroline $\mathbf{2 4}$, as a major product (Scheme 18).

It is well known that the transition metal-catalyzed intramolecular hydroamination reaction has become one of the most significant methods to access nitrogen-containing heterocycles. In particular, gold complexes have emerged as powerful catalysts for this purpose. As a general rule, most gold-catalyzed hydroaminations lead to the formation of the isoquinoline framework via 6-endo-dig cyclization, the corresponding 5-exo-dig product being unstable.

As reported by Fustero and coworkers, the hydroamination of fluorinated $o$ alkynylaryl carbamates $\mathbf{2 5}$ catalyzed by a cationic gold(I) complex evolved preferably to the isoindoline skeleton $\mathbf{2 6},{ }^{[21]}$ as opposed to the standard statements (Scheme 19). Moreover, the regioselectivity could be controlled and directed toward one major product by tuning the electronic property of the alkyne moiety.

Also, an electronic effect of the fluorinated group was observed since the gradual introduction of fluorine atoms at the $\alpha$-position A-F to the nitrogen promoted a 5-exo-dig mechanism, leading to isoindoline derivative $\mathbf{2 6}$ as the major product, in contrast to the published results with non-fluorinated substrates (Scheme 20). In some cases, 1,2-dihydroisoquinolines 27 arising from a 6-endo-dig mechanism were also detected.

Gouverneur and coworkers reported a de novo synthesis of a variety of racemic fluorinated carbohydrates using syn-glycal (士)-cis-29 as the key intermediate, which was in turn prepared by a gold-catalyzed cyclization as the key step (Scheme 21). ${ }^{[22]}$ Difluorinated ynone $\mathbf{2 8}$ was used as building block for this purpose, which underwent a selective 6-endo-dig cyclization in the presence of Gagosz' catalyst $\left.\left(\mathrm{Ph}_{3} \mathrm{PAuNTf}\right)_{2}\right)$, leading to the desired intermediate in good yield. An in-depth NMR investigation was carried out on the resulting gem-difluorinated hexose analogs to study the existence of intramolecular $\mathrm{C}-\mathrm{F} \cdots \mathrm{H}-\mathrm{O}$ hydrogen bonds.

\subsection{Palladium-catalyzed Reactions}

Despite widely-used fluorinated build-

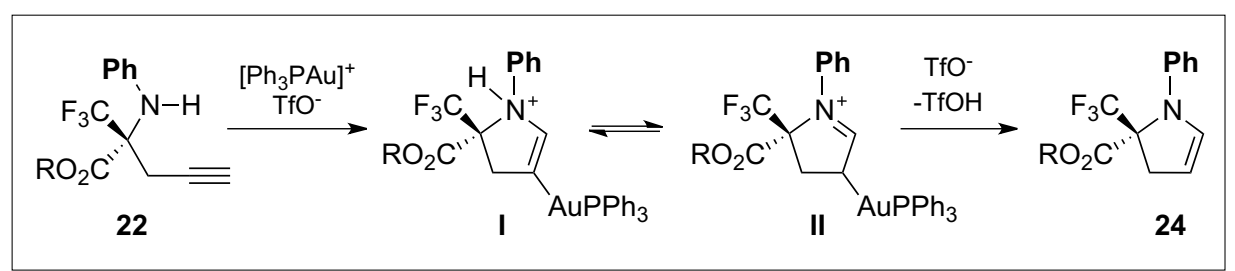

Scheme 18. Proposed mechanistic explanation.

ing blocks, new selective fluorination methods are continuously emerging in the literature. Herein, we focus on those catalyzed by palladium, among others. In this sense, the groups of Gouverneur and Brown described a palladium-catalyzed allylic fluorination reaction of properly functionalized substrates under mild conditions (Scheme 22).[23] The allylic leaving group resulted determining to yield 2-substituted propenyl fluorides $\mathbf{3 1}$ in high yields, as the traditionally used leaving groups in palla-

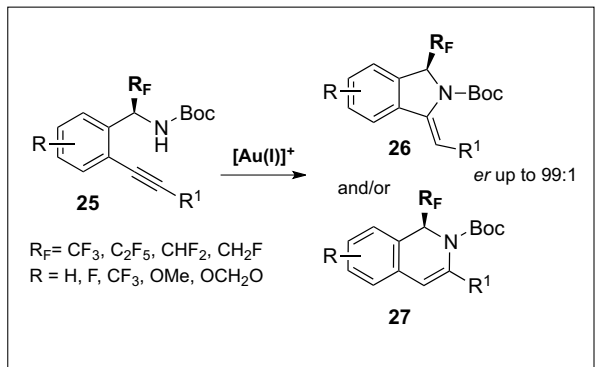

Scheme 19. Gold-catalyzed hydroamination of fluorinated o-alkynylaryl carbamates 25.

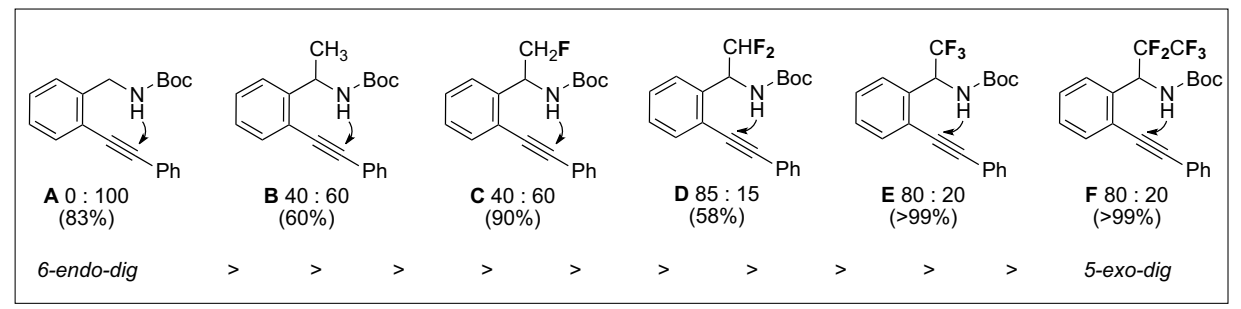

Scheme 20. $\alpha$-Substituent effect on the gold(I)-catalyzed cycloisomerization reaction.

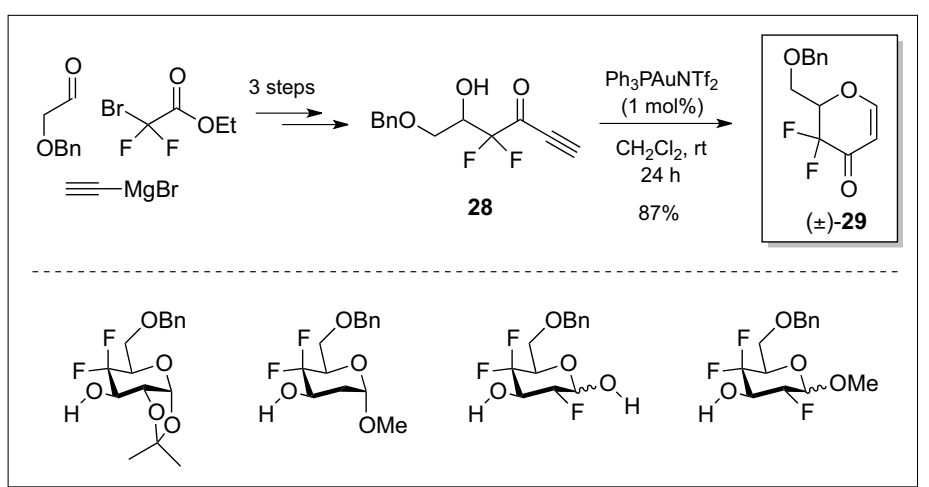

Scheme 21.

Preparation of the key intermediate 29 in the synthesis of racemic fluorinated carbohydrates.

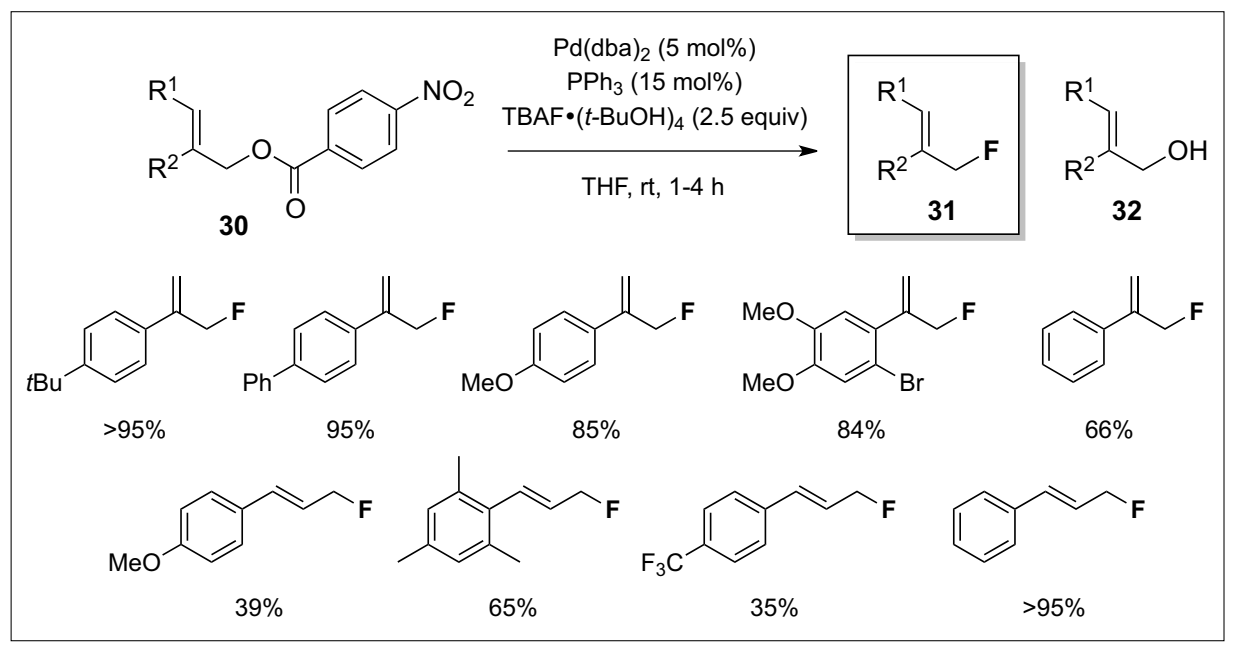

Scheme 22. Pd-catalyzed allylic fluorination reaction of $\mathbf{3 0 .}$ 
dium-catalyzed allylic alkylations, such as carbonates or acetates, led to the recovery of the starting material or form the allylic alcohol 32 under the reaction conditions. Thus, $p$-nitrobenzoate was unexpectedly employed as the leaving group of choice. Fluorination was then carried out in the presence of $5 \mathrm{~mol} \%$ of $\operatorname{Pd}(\mathrm{dba})_{2}$ and 15 $\mathrm{mol} \%$ of $\mathrm{PPh}_{3}$ at room temperature, using tetra- $n$-butylammonium tetra(tert-butyl alcohol)-coordinated fluoride [TBAF. $(t$ $\left.\mathrm{BuOH})_{4}\right]$ as a ' $\mathrm{F}$ '' source.

This methodology was also applied, for the first time, to the synthesis of $\left[{ }^{18} \mathrm{~F}\right]-$ radiolabeled compounds, which are widely employed in positron emission tomography (PET) experiments, and in oncology, despite their somewhat short half-life. Hence, as a first example of transition metal-mediated ${ }^{18} \mathrm{~F}$-fluorination, a palladiumcatalyzed allylic fluorination reaction of propenyl esters 33 was carried out using, in this case, $\left[{ }^{18} \mathrm{~F}\right] \mathrm{TBAF}$ as a fluorinating reagent in acetonitrile at room temperature (Scheme 23).
Also, Doyle and coworkers described an asymmetric version, the enantioselective synthesis of cyclic allylic fluorides 35 in just one step from the corresponding allylic chlorides 34 in the presence of a chiral biphosphine-ligated palladium(II) complex with AgF (Scheme 24).[24]

Experimental results supported a distinct mechanism for the C-F bond formation based on a $S_{N} 2$-type nucleophilic attack of fluoride on a Pd(II) $\pi$-allyl intermediate with an overall retention of the configuration (Scheme 25).

Its wide substrate scope, including silyl ethers and alcohols, makes this method very useful to prepare allylic fluorides with excellent enantioselectivities, having become an essential alternative to the dehydroxyfluorination with (diethylamino) sulfur trifluoride (DAST).

Later on, Doyle et al. extended this protocol to branched acyclic systems, developing a highly regio- and enantioselective synthesis of branched allylic fluorides $\mathbf{3 7}$ from allyl chlorides $\mathbf{3 6}$ and using $\mathbf{L 2}$
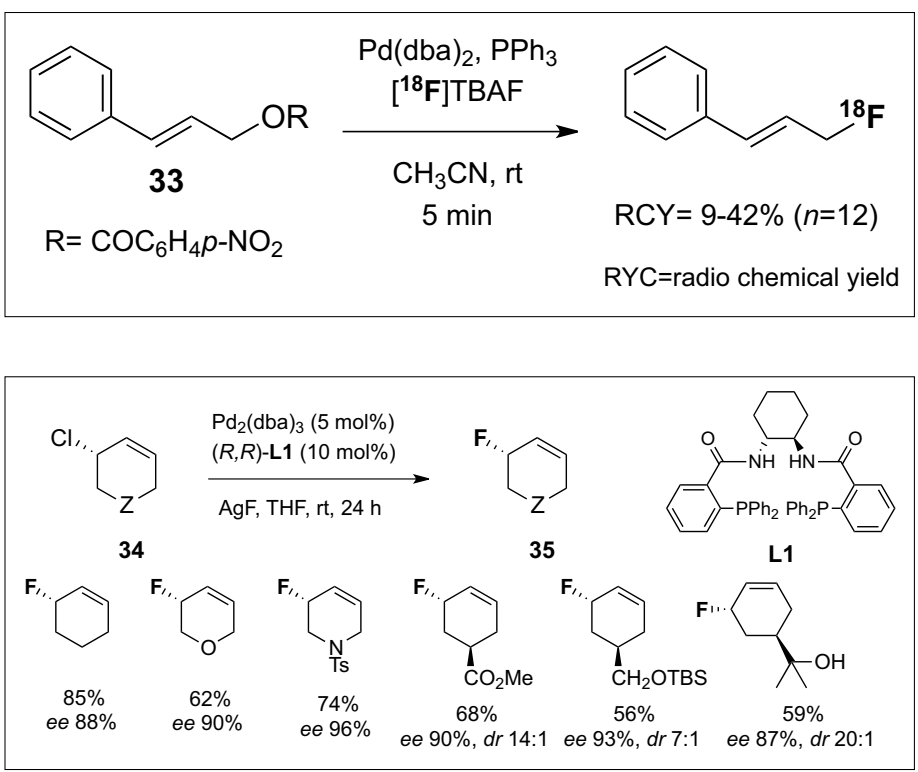

Scheme 24.

Enantioselective synthesis of cyclic allylic fluorides 35 .

Scheme 23. Pdcatalyzed allylic fluorination reaction of $\mathbf{3 3}$ by using $\left[{ }^{18} \mathrm{~F}\right] \mathrm{TBAF}$.

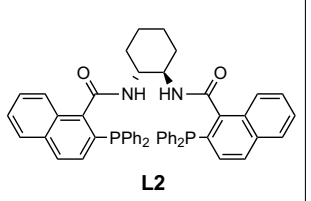

$$
\text { (2) }
$$

$84 \%$, ee $90 \%$
b: $l>20: 1$
(R,R)-L2 (10 mol\%)

$\mathrm{AgF}$, toluene
rt, $48 \mathrm{~h}$<smiles>[R]C1CCCCC1</smiles>

$\mathrm{R}^{\prime}{ }_{37}$

L2

$$
\text { } \begin{gathered}
84 \%, \text { ee } 58 \% \\
b: 1>20: 1
\end{gathered}
$$<smiles>C=CC(F)CCBr</smiles>

$66 \%$, ee nd
b.l $>20: 1$

$$
\begin{gathered}
50 \% \text {, ee } 90 \% \\
\text { b:l } 16: 1
\end{gathered}
$$

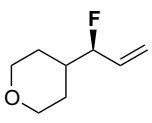

$62 \%$, ee $97 \%$

b:l $>20: 1$

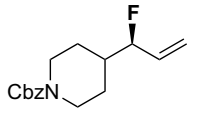
$88 \%$, ee $93 \%$
b:l $10: 1$

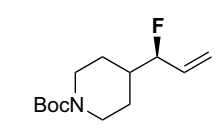

$85 \%$, ee $93 \%$, b:l 10: ${ }^{a}$ with DAST ee $49 \%$, b:I 2: $[b: l]=b r a n c h e d: l i n e a r$ selectivity; nd=non-determined

a Starting from $>99 \%$ ee branched allylic alcohol

Scheme 26. Selective synthesis of branched allylic fluorides $\mathbf{3 7 .}$ fluoride 37 . as chiral ligand (Scheme 26). ${ }^{[25]}$ As stated before, a Pd(II) $\pi$-allyl intermediate was involved in the catalytic cycle based on a nucleophilic attack of fluoride on this system.

Despite the tolerance with many functional groups, low enantioselectivities (ee $21-71 \%$ ) were observed for those less sterically hindered and no fluorination reaction occurred with secondary alkyl amines. By contrast, $\alpha$-branched allylic chlorides and bromides generated the corresponding fluorides with excellent regio- and enantioselectivity. Additionally, in the context of diversity-oriented synthesis, several transformations were carried out on the allylic fluoride $\mathbf{3 7}$ achieving access to a variety of optically pure fluorinated molecules whose synthesis would be unfeasible or comprise several steps (Scheme 27).

Thus far, several examples of fluorination reactions requiring a prefunctionalization of the substrate have been displayed. Nevertheless, the direct functionalization of $\mathrm{C}-\mathrm{H}$ bonds has recently emerged as a powerful tool in organic synthesis. [26] Synthetic methods for assembling $\mathrm{C}-\mathrm{F}$ bonds under mild conditions, even more so, at the final stage of the synthetic route, are of particular value. While $\mathrm{C}-\mathrm{H}$ fluorinations using electrophilic fluorinating reagents have been slightly more explored, those using nucleophilic fluoride are scarce. In this sense, Sanford and coworkers described a palladium-catalyzed benzylic fluorination reaction by $\mathrm{C}-\mathrm{H}$ bond activation of 8-methylquinoline derivatives $\mathbf{3 8}$ to obtain $\mathbf{3 9}$ in moderate to good yields (Scheme 28). ${ }^{[27]}$

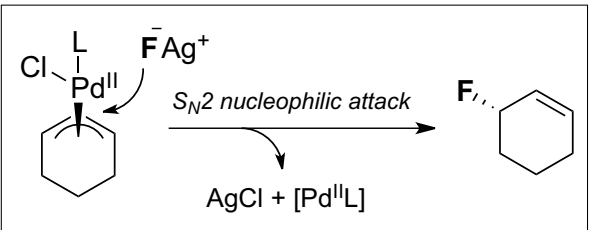

Scheme 25. Proposed mechanistic explanation.

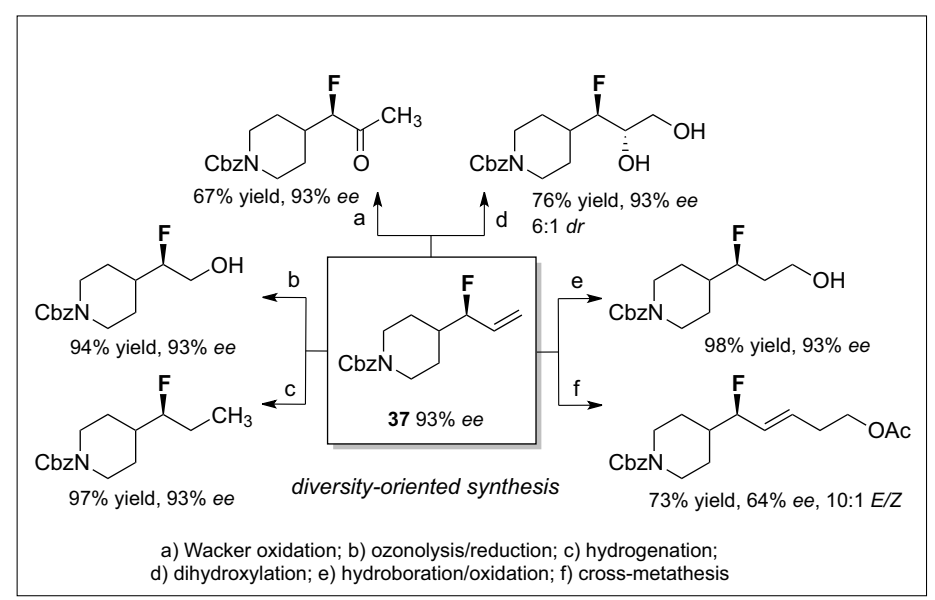

Scheme 27. Access to optically pure fluorinated molecules from allylic 


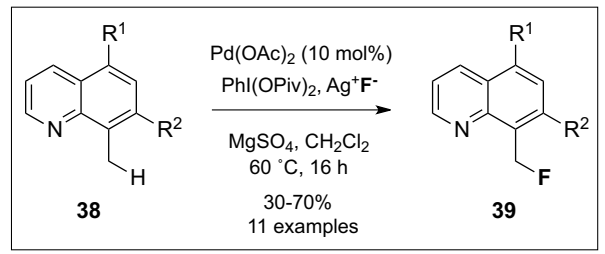

Scheme 28. Pd-catalyzed benzylic fluorination by $\mathrm{C}-\mathrm{H}$ bond activation of $\mathbf{3 8}$.

In general, fluorination took place in the presence of different substituents including halogens (e.g. Br, F or I), although chemical yields were not as good as desirable owing to the $\mathrm{C}-\mathrm{H}$ oxygenation side reaction. Sanford proposed an F-Pd(IV) complex as the key intermediate in the catalytic process but, neither the role of $\mathrm{Ag}^{+}$ion, whose presence was a requisite, in the presence of a phosphine-based ligand should be mentioned (Scheme 31). [29] Apparently, the phosphine ligand played an important role in the process as a Lewis base promoter as it seems that it cleaved the $\mathrm{H}-\mathrm{CF}_{3}$ bond to provide the $\mathrm{Pd}-\mathrm{CF}_{3}$ complex $\mathbf{4 2}$ which could participate further in aromatic trifluoromethylations.

In the field of arene fluorination, Ritter and coworkers have recently reported a similar reaction with aryl trifluoroborates catalyzed by an air- and moisture-stable terpyridyl palladium(II) complex (Scheme 32). ${ }^{[30]}$ This metal complex acted as a precatalyst since a rare palladium(III) species, which was further isolated and characterized by X-ray analysis, was in fact the catalyst in the fluorination reaction.

The trifluoroborates $\mathbf{4 3}$ could also be

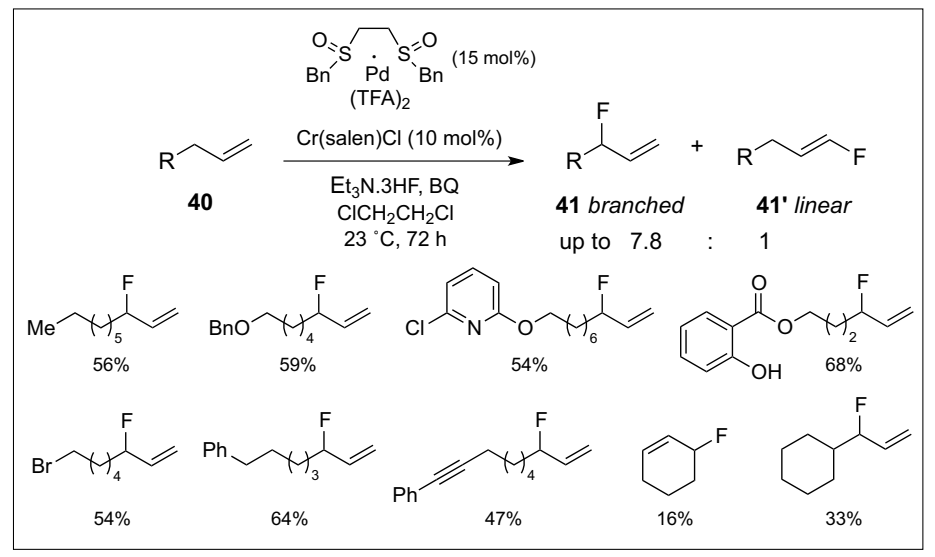

Scheme 29. Pd-catalyzed allylic C-H fluorination. prepared in situ from arylboronic acid derivatives $43^{\prime}$ by treatment with $\mathrm{NaF}$ and $\mathrm{KHF}_{2}$, giving the corresponding aryl fluorides $\mathbf{4 4}$ in excellent yields (Scheme 33 ). The mild reaction conditions used in this case were well tolerated by a wide range of functionalized aryl substrates apart from heterocycles. Furthermore, arene fluorination was highly regioselective, except for those compounds containing electronwithdrawing groups (e.g. amide), which gave constitutional isomers. The authors proposed a single-electron-transfer (SET) pathway as a plausible mechanism, involving a Pd(III) intermediate and radical species.

In line with this issue, a Pd-catalyzed difluoroalkylation of aryl boronic acids $\mathbf{4 5}$ has been reported very recently by Zhang and coworkers. ${ }^{[31]}$ To attach the difluoromethylene group $\left(\mathrm{CF}_{2}\right)$ to the arene ring, they employed readily available bromodifluoromethylated phosphonate, acetate and amides as fluorinated building blocks (Scheme 34). nor the identity of the active fluorinating reagent could be elucidated.

In this context, Doyle et al. developed distinct reaction conditions for their palladium-catalyzed allylic $\mathrm{C}-\mathrm{H}$ fluorination using a $\mathrm{Pd}(\mathrm{II})$-sulfoxide complex as catalyst, $\mathrm{Et}_{3} \mathrm{~N} \cdot 3 \mathrm{HF}$ as fluoride source and benzoquinone (BQ) as an external oxidant. ${ }^{[28]}$ The conversion of the allylic substrate $\mathbf{4 0}$ into 41 was markedly improved when a $\mathrm{Cr}$ (salen) $\mathrm{Cl}$ complex was used as catalytic additive. It should be noted that the addition of the fluorine atom was highly chemoselective for allylic position over other sensitive positions such as the propargylic or benzylic ones (Scheme 29).

Moreover, the authors applied this methodology to transform a polyfunctionalized steroid into its fluorinated analog, providing the desired molecule in good yield and regioselectivity (Scheme 30).

This process highlights the ready access to a wide range of fluorinated derivatives, for instance, from natural products, in a single operation under mild conditions without anhydrous requirements.

In the context of $\mathrm{C}-\mathrm{H}$ activation, the recent work by Grushin and Takemoto on the selective $\mathrm{H}-\mathrm{CF}_{3}$ activation with palladium

$$
\begin{aligned}
& {\left[\left(\mathrm{Ph}_{3} \mathrm{P}\right) \mathrm{Pd}(\mathrm{Ph})(\mu-\mathrm{OH})\right]_{2}} \\
& +\operatorname{dppp} \text { (2 equiv) } \overrightarrow{-2 \mathrm{PPh}_{3}}
\end{aligned}
$$

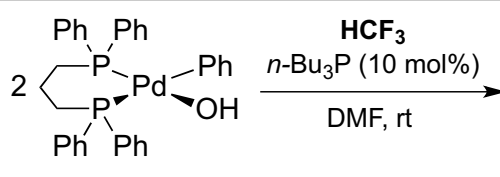

$$
\begin{aligned}
& 2 \overbrace{\mathrm{Ph}}^{\mathrm{Ph}} \mathrm{P}^{\prime} \mathrm{Ph}_{42}^{-} \mathrm{Ph}-\mathrm{CF}_{3}
\end{aligned}
$$

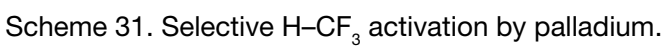

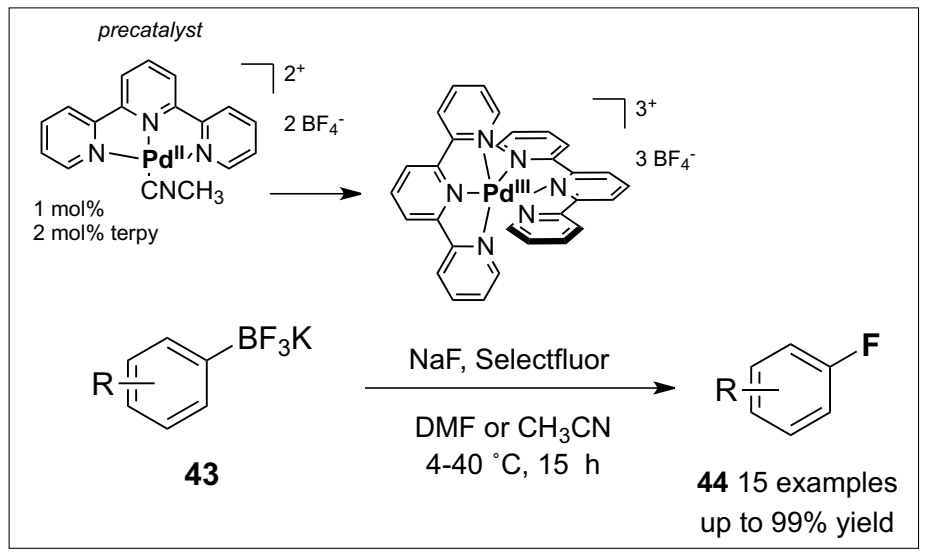

Scheme 32. Catalytic fluorination of aryl trifluoroborates.

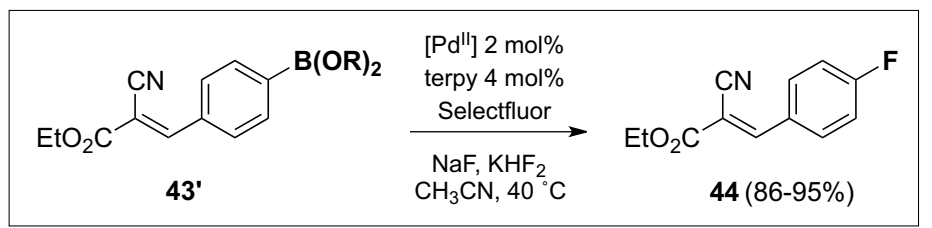

Scheme 33.

Fluorination of trifluoroborates prepared in situ from arylboronic acid 43'. 


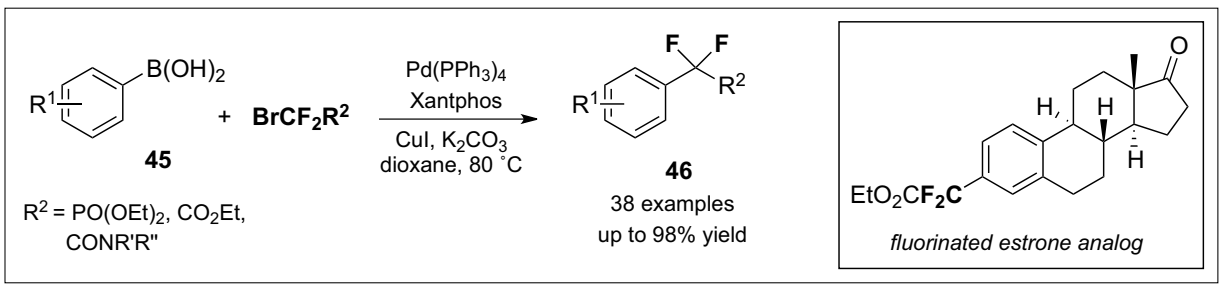

Scheme 34. Pd-catalyzed difluoroalkylation of aryl boronic acids $\mathbf{4 5}$.

Interestingly, only the Xantphos-Pd complex formed in the reaction media catalyzed the process efficiently, providing aryldifluoromethyl derivatives 46 in good to excellent yields. Moreover, this approach has been successfully applied to the late-stage synthesis of interesting bioactive compounds for drug discovery (Scheme 34). Although the mechanism has not been elucidated so far, the authors proposed the formation of difluoromethylene radicals through a Pd-promoted SET pathway as the initial step. This radical species would

$$
\begin{aligned}
& \mathrm{L}_{n} \mathrm{Pd}^{0}+\mathrm{BrCF}_{2} \mathrm{R}^{2} \stackrel{\text { SET }}{\longrightarrow} \mathrm{L}_{n} \mathrm{Pd}^{+}=\mathrm{BrCF}_{2} \mathrm{R}^{2} \longrightarrow \\
& \mathrm{L}_{\mathrm{n}} \mathrm{Pd} \mathrm{Br}^{\mathrm{B}}+\mathrm{CF}_{2} \mathrm{R}^{2} \longrightarrow \mathrm{R}^{2} \mathrm{CF}_{2} \mathrm{Pd}^{\mathrm{II}} \mathrm{L}_{\mathrm{n}} \mathrm{Br} \stackrel{\mathrm{ArB}(\mathrm{OH})_{2}}{\longrightarrow}
\end{aligned}
$$

Scheme 35. Proposed radical-based mechanism.

react with $\mathrm{L}_{\mathrm{n}} \mathrm{Pd}(\mathrm{I}) \mathrm{Br}$ generated in situ to provide the active catalyst which would be involved in the subsequent cross-coupling reaction (Scheme 35).

Leaving aside fluorination reactions, fluorine-containing compounds behave in a particular manner in the presence of palladium. That is the case of difluoropropargyl amides $\mathbf{4 7}$ synthesized for the first time by Hammond and coworkers ${ }^{[32]}$ and further used in the synthesis of difluoro $\beta$ - and $\gamma$-lactams by Fustero and coworkers. ${ }^{[33]}$ These small heterocycles constitute attractive scaffolds in total synthesis as well as new molecular entities in drug discovery. As mentioned before, Fustero, Hammond et al. reported a regioselective synthesis of four- and five-membered ring lactams via an intramolecular hydroamination reaction of difluoroamides 47 (Scheme 36).

The formation of $\gamma$-lactam 48 could be explained by a base-promoted nucleophilic addition of the amidic nitrogen to the electron-deficient triple bond in the presence of TBAF. In contrast, the formation of $\beta$-lactam 49 was observed in the presence of palladium acetate and $\mathrm{Et}_{3} \mathrm{~N}$ favoring a 4-exo-dig over 5-endo-dig mechanism as a result of the gem-difluoro moiety (Scheme 37).

Given the high number of methods for selective fluorination recently reported in the literature, ${ }^{[5]}$ the use of the resulting fluorides as appropriate substrates in subsequent transformations would be very interesting from a synthetic point of view. In this sense, Gouverneur and Brown explored the reactivity of benzylic fluorides $\mathbf{5 0}$ in palladium-catalyzed substitution and cross-coupling reactions in detail.[34] In that paper, the ability of fluoride as leaving group was disclosed even in the presence of other leaving groups such as acetate or carbonate. Based on their previous mechanistic experiments with allylic fluorides, ${ }^{[35]}$ Gouverneur and coworkers established suitable conditions for a palladium(0)-catalyzed nucleophilic substitution of bicyclic
Scheme 37. Formation of four-membered ring lactam 49. and monocyclic fluorides 50, compatible with different nucleophiles to obtain $\mathbf{5 1}$. The selective benzylic fluoride displacement over other functional groups tethered to the molecule is particularly noteworthy (Scheme 38).

Furthermore, the authors adapted the previous reaction conditions to SuzukiMiyaura cross-coupling of selected benzylic fluorides with phenylboronic acid. In that case, the regioselectivity of the process could be controlled by employing an appropriate phosphine ligand (Scheme 39).

It is well-known that the inclusion of fluorinated fragments, such as the trifluoromethyl group, in organic molecules has contributed significantly to the development of new pharmaceuticals. ${ }^{[36]}$ In this respect, the Ruppert-Prakash reagent $\left(\mathrm{TMSCF}_{3}\right)$ and its difluoromethyl variant $\left(\mathrm{TMSCF}_{2} \mathrm{H}\right)$, as ' $\mathrm{CF}_{3}$ ' and ' $\mathrm{CF}_{2} \mathrm{H}$ ' anion source respectively, have led to a substan-

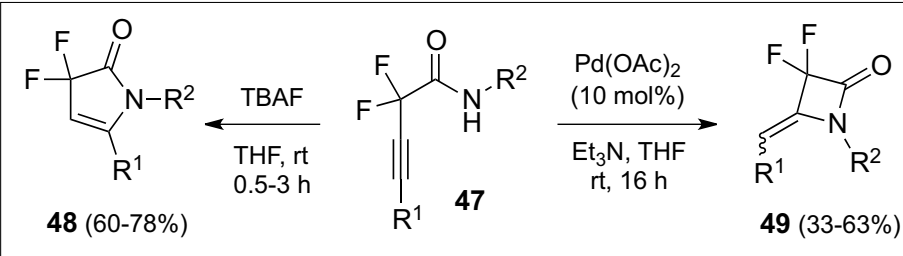

Scheme 36

Intramolecular hydroamination reaction of difluoroamides 47
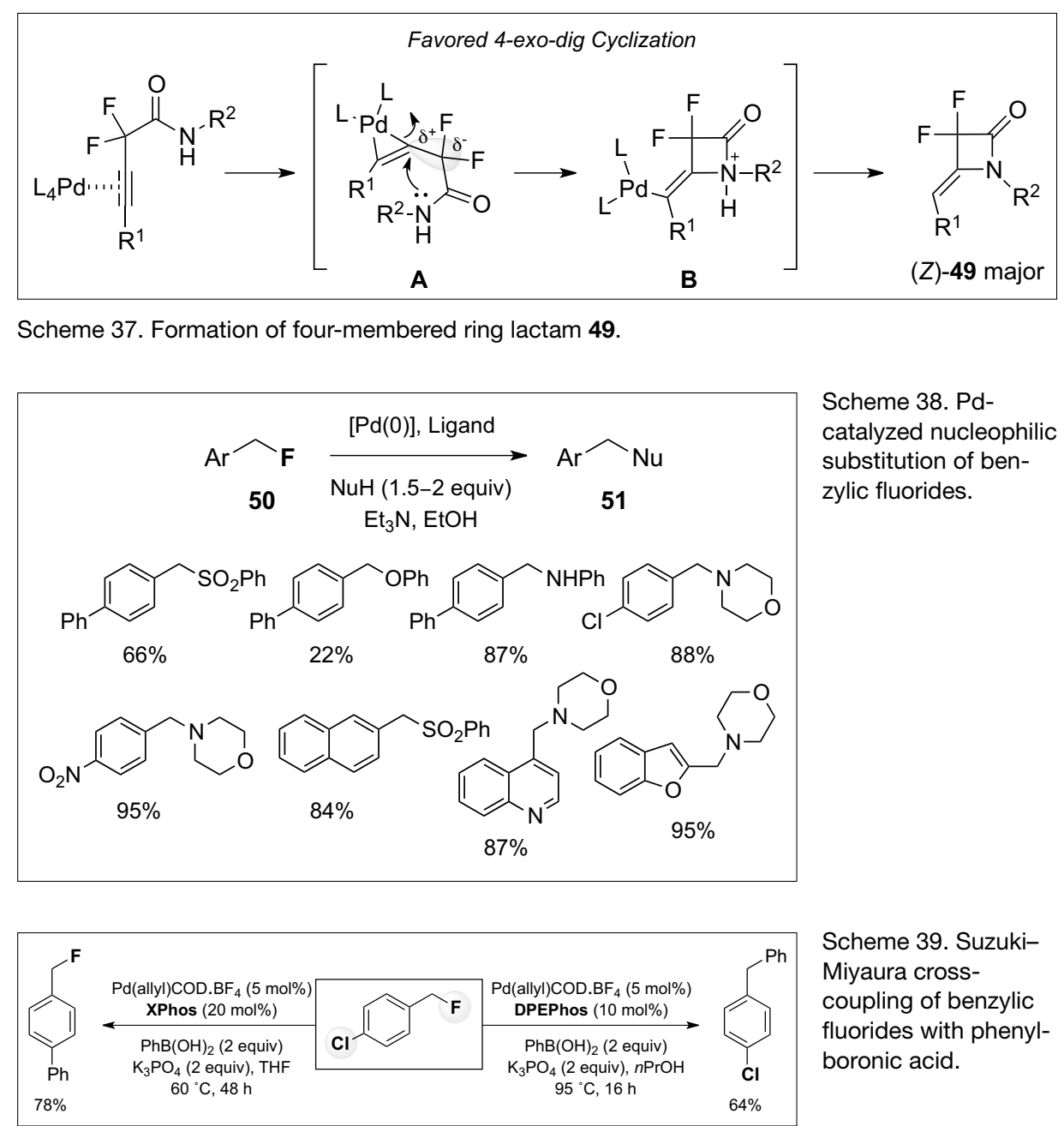

Scheme 39. SuzukiMiyaura crosscoupling of benzylic fluorides with phenylboronic acid. 
tial improvement in organic synthesis. However, the efficient incorporation of the trifluoroethyl moiety ' $\mathrm{CF}_{3} \mathrm{CH}_{2}$ ' had remained a challenge until recently. In 2012, Shibata and coworkers employed trifluoroethyl phenyl sulfones $\mathbf{5 2}$ and allyl carbonates $\mathbf{5 3}$ to provide trifluorohomoallylic sulfones $\mathbf{5 4}$ in excellent yields under mild conditions (Scheme 40). [37]

This approach constitutes an advantage over typical base-mediated allylation and involved a $\operatorname{Pd}(0)$-catalyzed decarboxylative allylation of $\mathbf{5 2}$ via a cationic $\pi$-allyl palladium complex intermediate. The ethoxy anion resulting from decarboxylation of allyl carbonate acts as a base deprotonating sulfone $\mathbf{5 2}$ and reacts further with the $\pi$-allyl palladium complex, regenerating the catalyst and leading to the desired product 54 (Scheme 41).

Furthermore, Shibata and coworkers explored the utility of these products which gave rise to the corresponding difluoromethyl derivatives $\mathbf{5 5}$ in high yields when they were subjected to reductive desulfonylation reaction (Scheme 42).

The difluoromethylene ' $\mathrm{CF}_{2}$ ' grouping is also an interesting structural moiety as it confers particular biological properties to organic molecules. ${ }^{[38]}$ Generally, the conversion of the carbonyl group with DAST or deoxofluor has been the method of choice to introduce the difluoromethylene group into organic molecules. However, a drawback of this method is its incompatibility with many functional groups. As an alternative, gem-difluoroallylation of boron-containing compounds $\mathbf{5 6}$ such as aryl and vinyl boronic acids, borates and trifluoroborates salts, has been described recently by Zhang and coworkers, involving a cross-coupling reaction catalyzed by palladium (Scheme 43). ${ }^{[39]}$ A simple protocol, wide functional group compatibility, very low catalyst loading $(0.4-0.01 \mathrm{~mol} \%)$ and high regioselectivity ( $\alpha / \gamma$ up to $>37: 1)$ are the advantages of this process.

\subsection{Silver-catalyzed Reactions}

Although silver has been known since antiquity, its use had mainly been limited to ornament manufacture and minting, in spite of its outstanding electrical conductivity and unique redox chemistry. However,

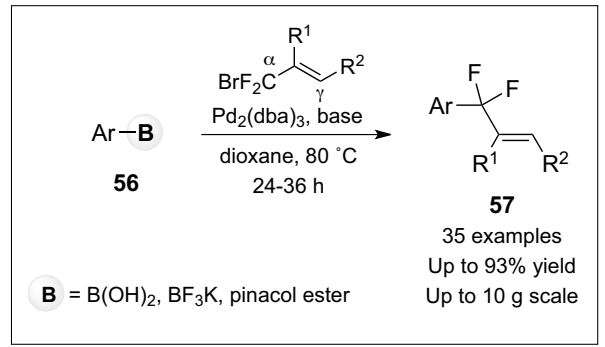

Scheme 43. gem-Difluoroallylation of boron derivatives 56 .

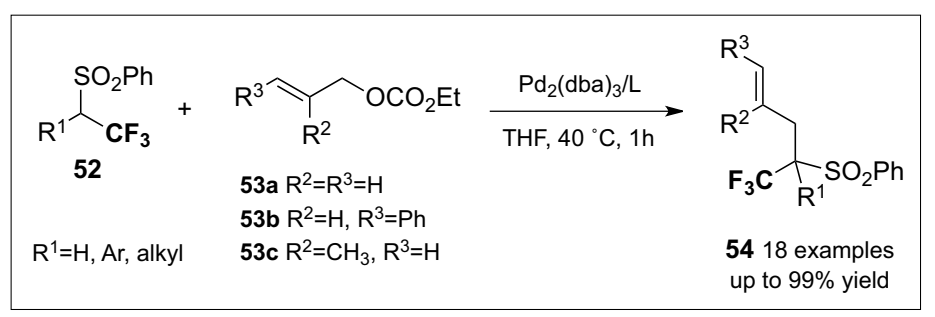

Scheme 40. Allylation of trifluoroethyl sulfones 52.

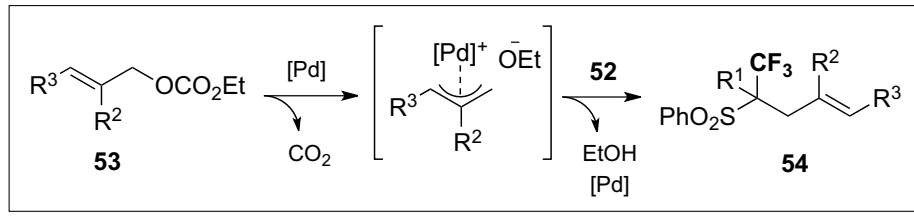

Scheme 41.

Proposed mechanism of Pd-catalyzed decarboxylative allylation.

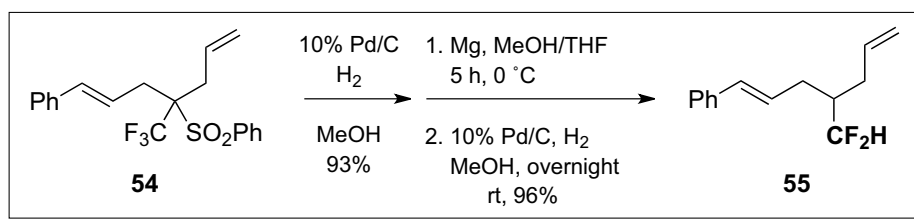

Scheme 42.

Reductive desulfonylation reaction.

owing to its ability to act as both $\pi$ and $\sigma$ Lewis acid, as well as the availability of its f orbitals, utilization of silver(I) in organic synthesis has gained significant popularity in the past several years. ${ }^{[40 \mathrm{a}-\mathrm{e}]}$

\subsubsection{Csp ${ }^{2}-F$ Bonds}

Due in part to their unique physicochemical and biological properties, aryl fluorides have found increased applications in fields of pharmaceutical, agrochemical and material sciences. ${ }^{[11]}$ The direct formation of $\mathrm{C}(\mathrm{Ar})-\mathrm{F}$ bonds has remained an important challenge for chemists and new and mild methods for direct incorporation of fluorine allowing a broader functional group tolerance are still ardently soughtafter.

In recent years silver-mediated/catalyzed transformations have emerged as powerful tools for the construction of such an important functionality. As outlined by Ritter and coworkers, ${ }^{[42]}$ aryl fluorides 59 can be prepared in good yields starting from functionalized aryl tri- $n$-butyl stannanes 58a and the fluorinating reagent F-TEDA$\mathrm{PF}_{6}(\mathbf{6 0})$, a derivative of Selectfluor. The authors later expanded their methodology to the less toxic arylboronic acids $\mathbf{5 8 b ^ { [ 4 2 b ] }}$ with complete suppression of the difficult- to-separate proto-demetalated product obtained with the use of aryl stannanes. In the presence of a base, aryl boronic acids and esters were shown to transmetalate to silver in a methanolic solution; subsequent reaction with Selectfluor (61) in acetone afforded aryl fluorides 59 in a one-pot fashion in good yields (Scheme 44, part I). Unlike the metal-free fluorination of alkenylboronic acids and trifluoroborates, ${ }^{[43]}$ which usually gives a $1: 1$ ratio of $E / Z$ isomers, ${ }^{[44]}$ transmetalation from boron 62 to silver occurs in a stereospecific way, thus providing complete control of the stereochemistry in the preparation of vinyl fluorides 63 (Scheme 44, part II).

Following the initial discovery of silver-mediated fluorinations, a catalytic fluorination of aryl stanannes 58a was developed. ${ }^{[42 c]}$ Unproductive protodestannylation products were suppressed by addition of $\mathrm{NaHCO}_{3}$ (2 equiv) and NaOTf ( 1 equiv), thereby making the use of 5 mol\% of $\mathrm{Ag}_{2} \mathrm{O}$ and 1.5 equiv of $\mathbf{6 0}$ possible (Scheme 45, part A). Mechanistic investigations suggested a $\mathrm{Ag}(\mathrm{I})-\mathrm{Ag}(\mathrm{II}) \mathrm{re}-$ dox cycle. Oxidation of a species (ArAg). (AgOTf) with reagent 60 led to a bimetallic $\mathrm{Ag}$ (II) intermediate which is responsible for the carbon-fluorine bond formation,

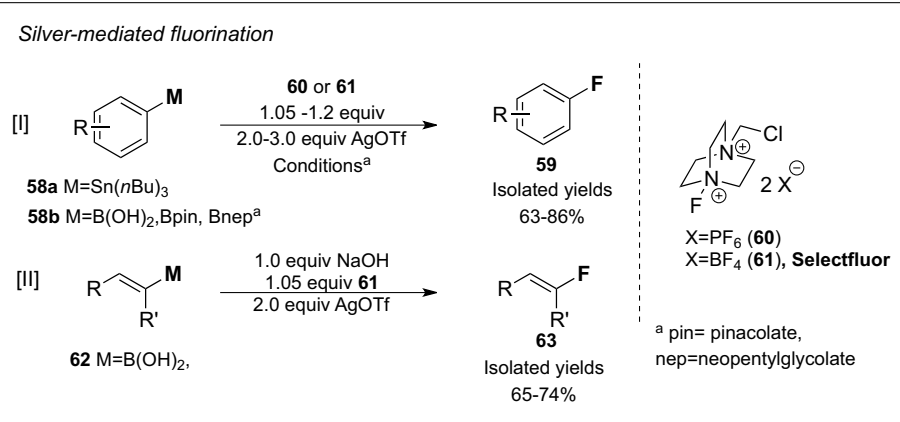

Scheme 44. Silvermediated fluorination of aryl nucleophiles and vinylboronic acids. 
a mechanism that differs from traditional cross-coupling mechanisms with other transition metals (Scheme 45, part B). Hitherto, silver-catalyzed fluorinations of boron nucleophiles have not been reported.

\subsubsection{Csp ${ }^{3}-F$ bonds}

A substantial achievement in the construction of $\mathrm{C} s p^{3}-\mathrm{F}$ bonds not restricted to the synthesis of $\alpha$-fluoro carbonyl compounds ${ }^{[45]}$ was reported by $\mathrm{Li}$ and coworkers in 2012. ${ }^{[46]}$ Catalytic amounts of $\mathrm{AgNO}_{3}$ were shown to promote decarboxylative fluorination of aliphatic carboxylic acids 64 by Selectfluor (Scheme 46). It was suggested, that an $\mathrm{Ag}(\mathrm{III})-\mathrm{F}$ species I formed in situ from $\mathrm{F}^{+}$and the $\operatorname{Ag}(\mathrm{I})$ catalyst was reduced to an $\mathrm{Ag}$ (II)-F species II after a SET by a carboxylate anion, thus releasing $\mathrm{CO}_{2}$ and the alkyl radical $\mathrm{R}$, which was later trapped by the $\mathrm{Ag}(\mathrm{II})-\mathrm{F}$ species, regenerating the $\operatorname{Ag}(\mathrm{I})$ catalyst and forming the alkyl fluoride $\mathbf{6 5}$, thus closing the catalytic cycle. A metal-free radical decarboxylative fluorination had been known from the corresponding $t$-Bu peresters by NFSI, ${ }^{47]}$ but the required extra step for their preparation was avoided in Li's protocol. Furthermore, the method made site-selective $\mathrm{Cs} p^{3}-\mathrm{F}$ bond formation in aqueous media and with excellent chemoselectivity towards aliphatic carboxylic acids possible. Under these conditions, the reactivity of the substrates was in the order of $3^{\circ}>2^{\circ}>1^{\circ}$, while aromatic carboxylic acids were inert, thus, allowing the preparation of substrate 66a in good yield. The authors demonstrated the scope of the process in the preparation of two biologically relevant fluorinated steroids 66b (Scheme 46).

\subsubsection{Synthesis of $\mathrm{CF}_{3}$-(Hetero)aro- matic Compounds}

Direct $\mathrm{C}-\mathrm{H}$ trifluoromethylation of aromatics can be achieved by means of a reaction of arenes and heteroarenes with silver trifluoroacetate ${ }^{[48]}$ in the presence of $\mathrm{TiO}_{2}$ as a photocatalyst. In this transformation, the photochemical decarboxylation of $\mathrm{CF}_{3} \mathrm{CO}_{2} \mathrm{Ag}$ on the surface of excited $\mathrm{TiO}_{2}$ generated ${ }^{\circ} \mathrm{CF}_{3}$ radicals, which underwent radical addition to the aromatic substrates, thus affording trifluoromethyl aromatics $\mathbf{6 7}$ as a mixture of regioisomers (Scheme 47).

A silver-mediated direct $\mathrm{C}-\mathrm{H}$ trifluoromethylation has also been accomplished by means of a AgOTf/TMSCF $/ \mathrm{KF}$ system. In this fashion, Sandford and coworkers ${ }^{[49]}$ were able to afford trifluoromethylated products by using an excess (20 equiv) of the arene, 4 equiv of AgOTf, 4 equiv of $\mathrm{KF}$ and $\mathrm{TMSCF}_{3}$ (1 equiv) (Scheme 47). Noticeably, iodobenzene afforded a nonipso-substituted product, thus contrasting with the $\mathrm{Cu}$-catalyzed/mediated procedures (vide infra). Moreover, the fact that

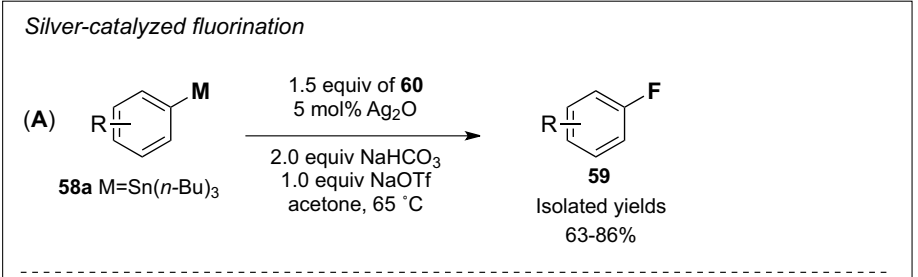

Scheme 45. Silvercatalyzed fluorination of aryl stannanes and mechanistic hypothesis.

Mechanistic hypothesis

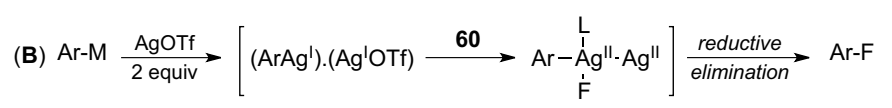

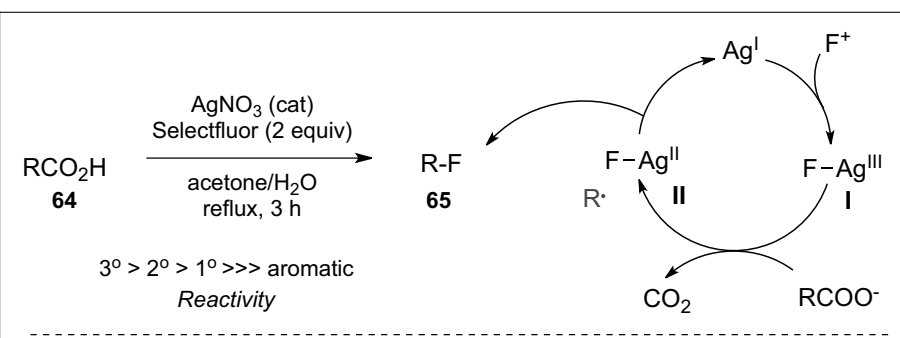

Scheme 46. Decarboxylative fluorination of aliphatic carboxylic acids.
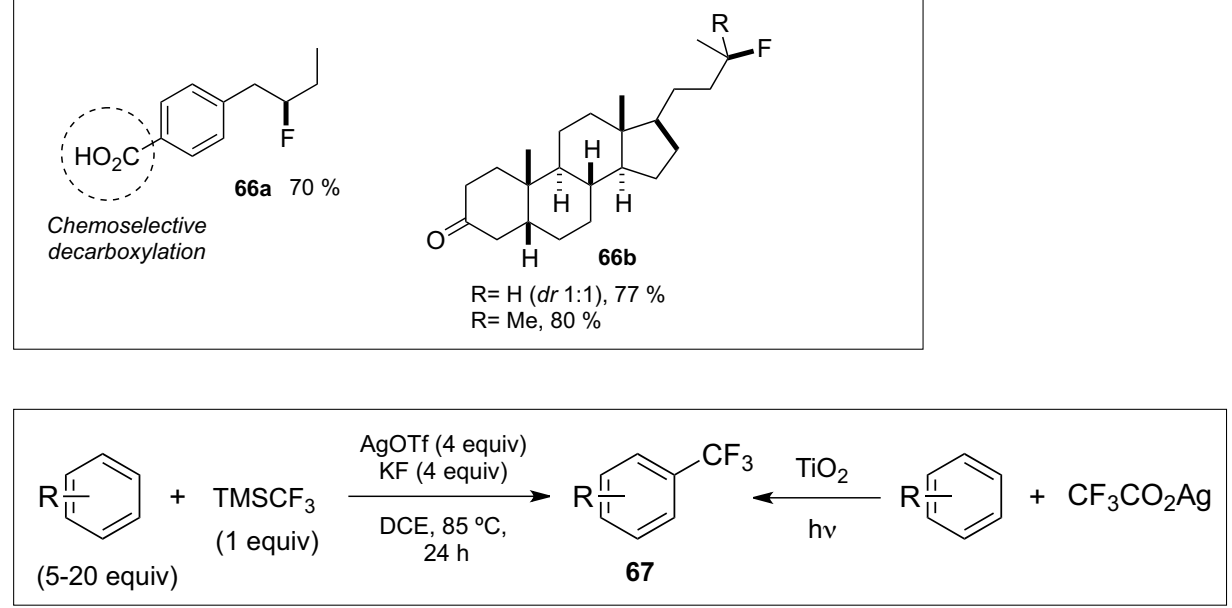

Scheme 47. C-H trifluoromethylation of arenes. regioisomers were obtained and that the reaction was suppressed in the presence of TEMPO, lent further support to the intermediacy of $\mathrm{a}^{\circ} \mathrm{CF}_{3}$ radical, possibly from $\mathrm{AgCF}_{3}$.

Very recently, Bräse and coworkers ${ }^{[50]}$ disclosed a procedure for the ortho-selective $\mathrm{C}-\mathrm{H}$ functionalization of aromatics. By means of a triazene-directing group 68, they were able to achieve direct trifluoromethylation, perfluoroethylations, heptafluoropropylation and ethoxycarbonyldifluoromethylation of aromatics 69. In this transformation, the $\mathrm{AgR}_{\mathrm{F}}$ species was gen- erated in situ from TMS- $\mathrm{R}_{\mathrm{F}}$ and $\mathrm{AgF}$ using perfluorohexane as solvent. Notably, other donor solvents commonly used in trifluoromethylations such as DMF led to a number of by-products and/or decreased yields. In some cases, the di-ortho $\mathrm{R}_{\mathrm{F}}$-substituted products $\mathbf{7 0}$ where observed, albeit in lower yields. Salient features of this process include: a remarkably high site-selectivity towards ortho-trifluoromethylated products as well as the possibility to further transform the triazene moiety, thus making this a highly attractive method for direct $\mathrm{C}-\mathrm{H}$ trifluoromethylation (Scheme 48).

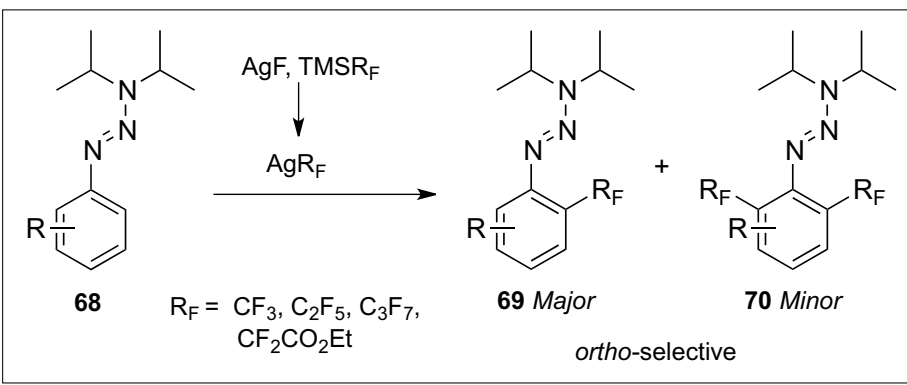

Scheme 48. Triazenedirected orthotrifluoromethylation of aromatics. 
A novel approach for the synthesis of benzotrifluorides $\mathbf{7 1}$ was recently introduced by Wang and coworkers. ${ }^{[51]}$ Starting from $\mathrm{AgCF}_{3}$ and readily available aromatic amines, trifluoromethyl arenes were obtained after the one-pot diazotization reaction with the $t$-BuONO/HCl(aq) system. After the generation of the corresponding diazonium chloride in an EtCN solution, 3.5 equiv of previously prepared $\mathrm{AgCF}_{3}$ were added at low temperature $\left(-78^{\circ} \mathrm{C}\right)$. Interestingly, the more stable and isolable tetrafluoroborate salts were less reactive under their optimized reaction conditions. This transformation tolerates a variety of functional groups, from electron-donor, electron-withdrawing, alkynyl, vinyl and even to boron pinacolates (Scheme 49). Furthermore, a number of benzofurans and indoles were smoothly converted into the corresponding $\mathrm{CF}_{3}$-substituted products. However, 3-aminopyridine afforded only a $10 \%$ yield. Notably, ortho-substituted starting materials were also suitable substrates, thus, the corresponding products were obtained in high yields. Similar reports that use the $\mathrm{CuCF}_{3}$ species have recently emerged (vide infra).

\subsubsection{Electrophilic/Radical Fluorination/Fluoroalkylation of $\mathrm{C}-\mathrm{C}$ Multiple Bonds \\ 2.4.4.1 Reactions with Allenes and Alkynes}

In recent years, transition metal-catalyzed cyclization reactions have gained popularity for the fast and efficient synthesis of a number of biologically relevant heterocycles[40b] starting from readily available starting materials. ${ }^{[52]}$

In a series of reports, Liu's group ${ }^{[53]}$ disclosed a novel tandem approach for the synthesis of fluorine-containing heterocycles. With the use of $20 \mathrm{~mol} \%$ $\mathrm{AgNO}_{3}$ and the electrophilic fluorinating reagent $N$-fluoro-bis(benzenesulfonyl) amine (NFSI), fluorinated dihydropyrroles 73 (Scheme 50), and isoquinolines 76 (Scheme 51) were assembled, starting from $N$-tosyl-protected allenes $\mathbf{7 2}$ and $N$-substituted- $O$-alkynyl benzaldimines $\mathbf{7 5}$, respectively.

Intramolecular aminofluorination of the starting materials led to vinyl-Ag intermediate I, which was further oxidized by NFSI to afford the bimetallic $\mathrm{Ag}$ (II) fluoride intermediate II and a vinyl fluoride upon reductive elimination of the $\mathrm{C}-\mathrm{F}$ fragment. The obtained dihydropyrroles $\mathbf{7 3}$ were successfully aromatized to afford the fluoro-pyrrol derivatives $\mathbf{7 4}$ in good yields (Scheme 50).

Fluorinated isoquinolines $\mathbf{7 6}$ were readily obtained from the aminofluorination of $N$ - $t$-Bu- $o$-alkynyl benzaldimines 75 with 1.5 equiv of NFSI with concomitant elimination of isobutylene (Scheme

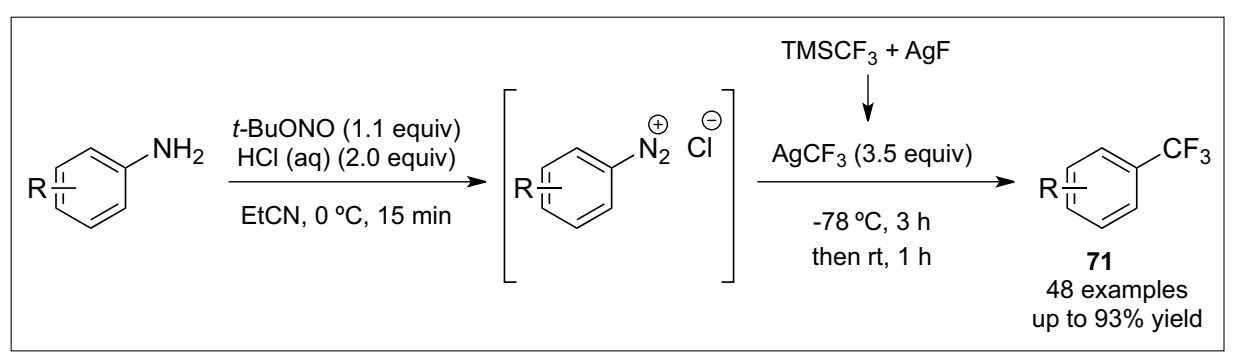

Scheme 49. Sandmeyer-type trifluoromethylation with $\mathrm{AgCF}_{3}$.

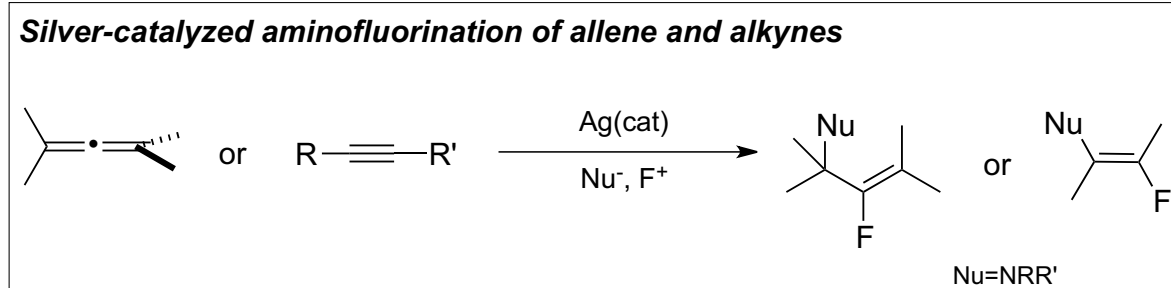

Silver-catalyzed aminofluorination of allenes

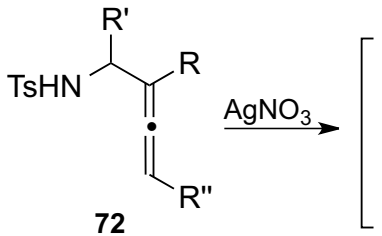

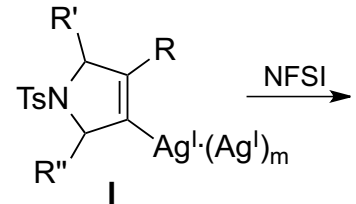<smiles>[R]C1=C([Al]2CCC(C)C2)C([R])N([As])C1[R]</smiles><smiles>[R]C1=C(F)C([R7])N([Te]C)C1[R]</smiles><smiles>[R]c1[nH]c([R])c(F)c1[R]</smiles>

$28-92 \%$

21 examples

$60-89 \%$

8 examples

Scheme 50. Silver-catalyzed synthesis of pyrrol derivatives.

51 , path 1). In the presence of 3 equiv of $\mathrm{Li}_{2} \mathrm{CO}_{3} 5$ equiv of NFSI, suitably substituted $o$-alkynyl benzaldimines were shown to provide 1,3 dipolar intermediates of type III, which were later trapped with dimethyl acetylene dicarboxylate 77a in a [3+2] dipolar cycloaddition reaction. In this transformation an oxazolidine-based bidentate ligand L1 (Scheme 51) was proved to be essential to promote product formation. The pyrrolo $(\alpha)$ isoquinolines generated from this double tandem process were achieved in good yields. Furthermore, the presence of a $\mathrm{CF}_{3}$ group in the dipolarophile 77b afforded $\mathbf{7 8}$ in good yields and with excellent regioselectivity, thus demonstrating the usefulness of the $\mathrm{CF}_{3}$ group as a tool to dictate the stereochemical outcome of these transformations (Scheme 51, path 2).

In a related report, the $N$-alkylated isoquinolinium species IV derived from the fluorination protocol was trifluoromethylated to afford 1-(trifluoromethyl)- 4-fluoro-1,2-dihydroisoquinolines 79. The success of the reaction with an $N$-alkyl substituent as opposed to the $t$-butyl substituent was explained in terms of the thermal stability of the resulting isoquinolinium species. In this report, a bidentate nitrogen ligand L2 (Scheme 51) promoted the trifluoromethylation with the RuppertPrakash reagent $\mathrm{TMSCF}_{3}$ to afford 79 in good yields (Scheme 51, path 3).

Recently, You and coworkers[54] combined a tandem cyclization approach and electrophilic fluorination for the synthesis of 3,3-difluoro-3H-indoles 81, 3-fluoroindoles 82 and 2-subtituted-3,3-difluoroindolines $\mathbf{8 3}$ starting from readily available $o$-alkynylanilines. The combination of a silver-catalyzed cyclization and electrophilic fluorination with NFSI or Selectfluor provided fluorinated indole derivatives in a single operation efficiently and in good yields (Scheme 52). These tandem and one-pot procedures are regarded as highly efficient, avoiding the need for purification 
of the corresponding intermediates. In this transformation, the 3-fluoro-indole derivatives 82 were isolated in modest yields, possibly due to the over-oxidation by the $\mathrm{F}^{+}$source.

A related silver-mediated process disclosed by Hammond and coworkers ${ }^{[55]}$ involves the cyclization-triggered addition of the Ruppert-Prakash reagent to aminoalkynes under microwave irradiation. The presence of 1.5 equiv of $\mathrm{AgF}$ enabled the one-pot preparation of $\alpha$-trifluoromethyl amines 85 of varying ring sizes in short reaction times. Most likely, the real nucleophilic species is the in situ generated $\mathrm{AgCF}_{3}$. Deuterium labeling experiments suggested that this species underwent nucleophilic addition to a reversibly formed enamine intermediate I. Accordingly, in the absence of a second nucleophile, intermediate I, generated after the initial alkyne hydroamination of $\mathbf{8 4}$, was detected but could not be isolated. The role of silver in this transformation, apart from the generation of $\mathrm{AgCF}_{3}$, was likely to be the alkyne activation for the intramolecular hydroamination step and the importance of a second nucleophilic species for the reaction was highlighted (Scheme 53). Noticeably, the reaction worked well not only with $\mathrm{CF}_{3}$ as nucleophile, but excellent yields were also obtained when TMSCN was used under copper catalysis affording $\alpha$-cyano-amines, thus, the broad nature of this alkyne double addition concept was showcased.

\subsubsection{Reactions with Alkenes}

In recent years, radical fluoro-functionalization of alkenes has emerged as a powerful strategy for the efficient preparation of fluorine-containing molecules. Hydrofluorination, carbofluorination, oxyfluorination are among the methods of particular interest, and more recently, methods for the aminofluorination and phosphonofluorination have appeared in the literature (Schemes 54-58). Noteworthy, silver catalysis has been a crucial component in many of these transformations, in which other transition metals were unable to bring about product formation.

A significant achievement for the synthesis of $\alpha-\mathrm{CF}_{3}$-ketones from olefin starting materials and radical $\mathrm{CF}_{3}$ sources was reported independently by $\mathrm{Xiao}^{[56]}$ and Maiti. [57] In Xiao's report, styrene derivatives were transformed into the corresponding $\alpha$-trifluoromethyl ketones $\mathbf{8 7}$ under metal-free conditions, albeit in low yields. In Maiti's methodology, yields and functional group tolerance were substantially improved with the aid of silver catalysis. Accordingly, $\alpha$-trifluoromethyl ketones were obtained not only from styrenes but also from heteroaromatics, as well as vinyl cycloalkane starting materials. The

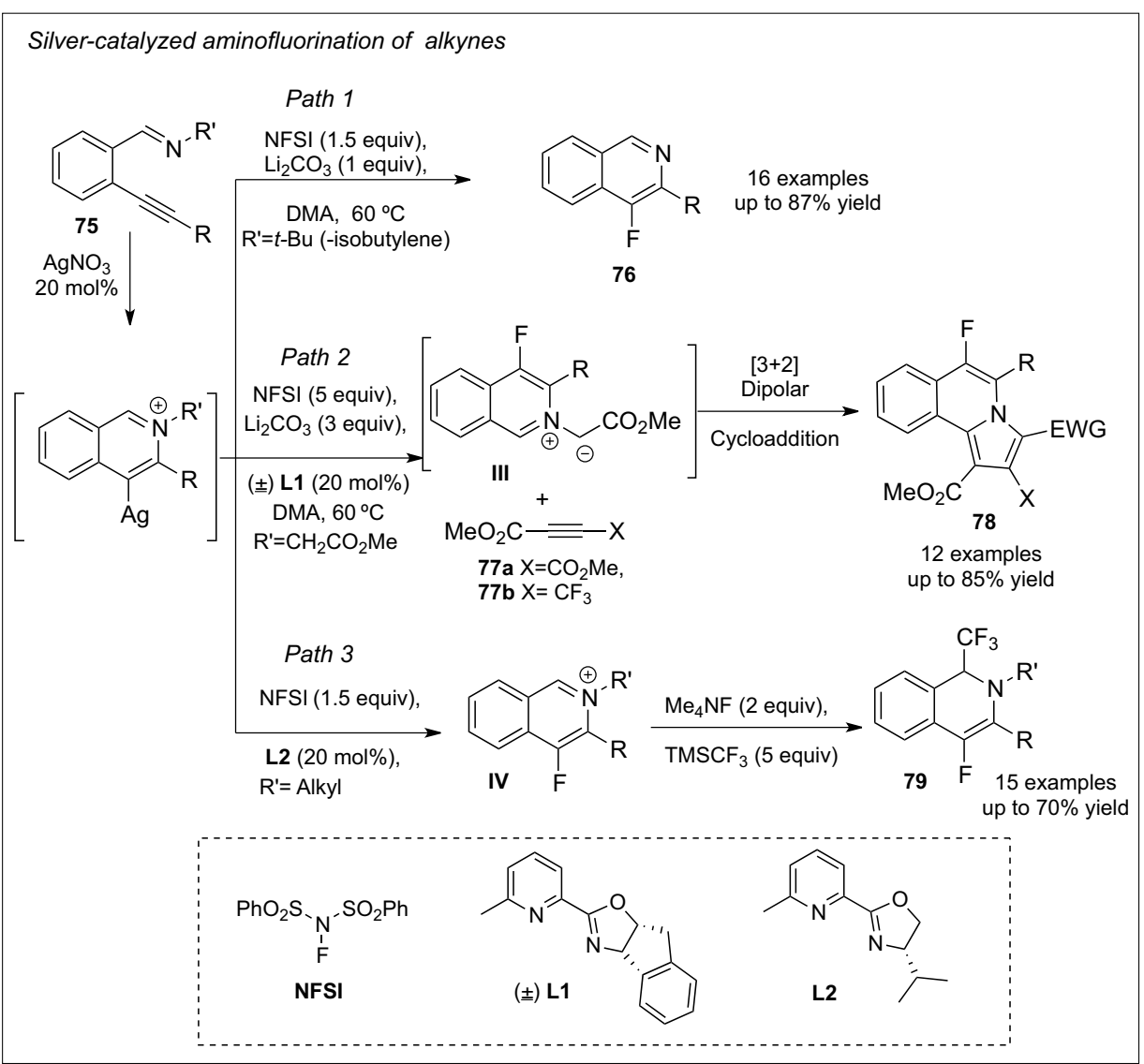

Scheme 51 . Silver-catalyzed synthesis of isoquinolines, pyrrolo $(\alpha)$ isoquinolines and trifluoromethyl-substituted dihydroisoquinolines.
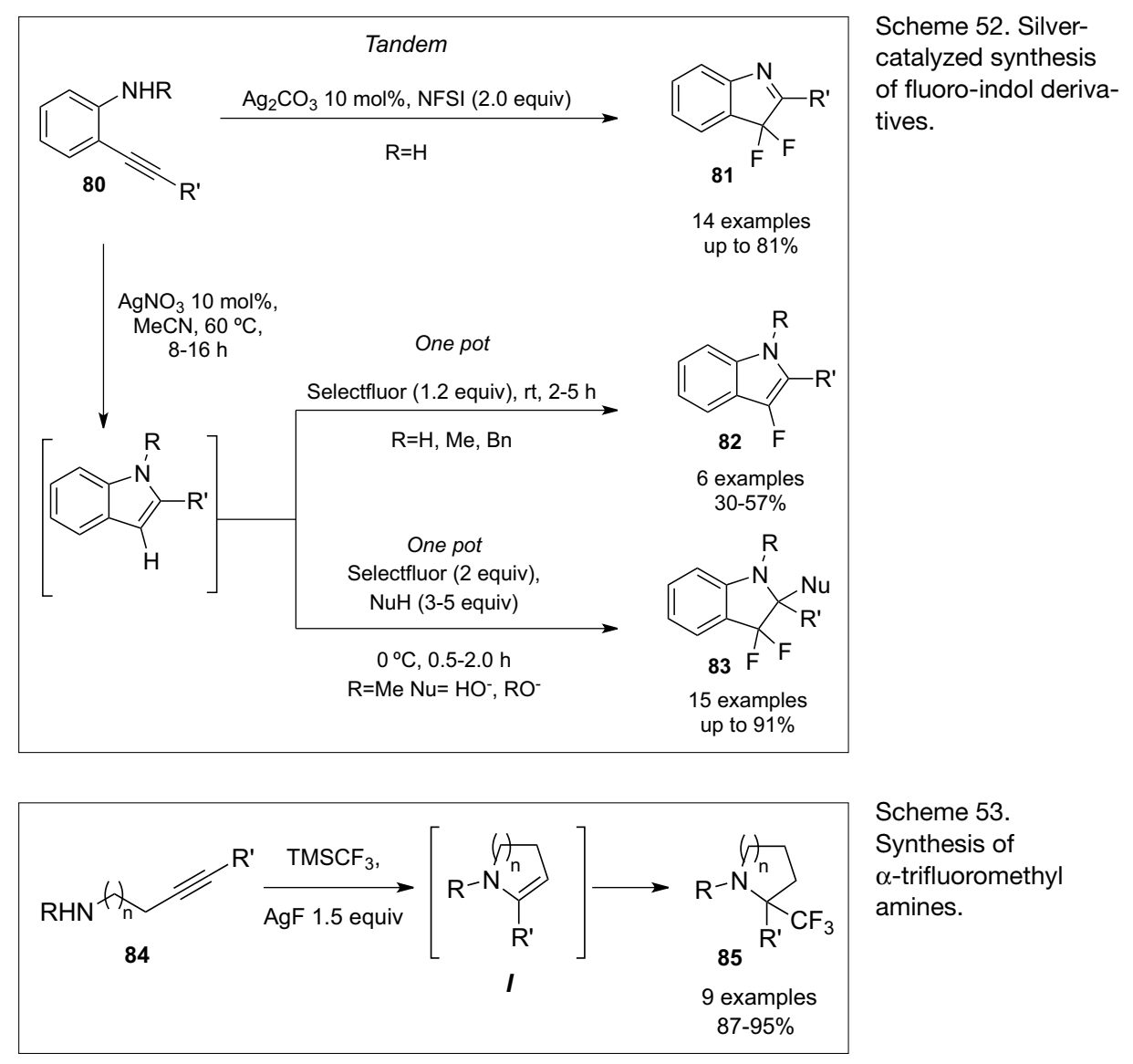

use of the inexpensive Langlois reagent $\mathrm{CF}_{3} \mathrm{SO}_{2} \mathrm{Na}$, room temperature and open-air conditions makes this method particularly attractive. Catalytic amounts of potassium persulfate $\mathrm{K}_{2} \mathrm{~S}_{2} \mathrm{O}_{8}$ as an external oxidant in conjunction with $20 \mathrm{~mol}_{\%} \mathrm{AgNO}_{3}$ under air 
afforded the corresponding products $\mathbf{8 7}$ in excellent yields (Scheme 54). Mechanistic investigations suggested the involvement of an $\alpha$-trifluoromethyl benzylic radical species $\mathbf{I}$, generated by the reaction of a $\mathrm{CF}_{3}$ radical with the olefin, which was later trapped by persulfate or dioxygen (as determined by ${ }^{18} \mathrm{O}$-labeling experiments). Owing to the exceptionally mild reaction conditions, functional groups such as ester, keto, aldehyde, nitro and cyano were tolerated as well as the easily oxidized chloromethyl group (Scheme 54).

A similar fluoro-functionalization of olefins was reported in 2013 by $\mathrm{Li}$ and coworkers, ${ }^{[58]}$ in which silver catalysis played an essential role in the radical aminofluorination of unactivated alkenes in aqueous media. Through the reaction of the corresponding substituted enamides 88 with 2 equiv of Selectfluor as the fluorine atom source and $10 \mathrm{~mol} \%$ of $\mathrm{Ag}(\mathrm{I})$ salts, a range of fluorinated $N$-substituted pyrrolidin-2-ones, oxazolidin-2-ones and $\mathrm{N}, \mathrm{N}$-disubstituted imidazolidin-2-ones (89, $\mathrm{X}=\mathrm{C}, \mathrm{O}$ and $\mathrm{N}$, respectively) was prepared (Scheme 55). The authors surmised that a radical intermediate II generated upon reaction of an amidyl radical and the double bond, was quenched by a fluorine atom from a $\mathrm{Ag}$ (II)-F generating the aminofluorination product $\mathbf{8 9}$ and regenerating the $\operatorname{Ag}(\mathrm{I})$ catalyst. A number of $\operatorname{Ag}(\mathrm{I})$ salts such as $\mathrm{AgNO}_{3}, \mathrm{AgOTf}$ and $\mathrm{AgOAc}$ in a $10 \mathrm{~mol} \%$ loading could efficiently catalyze this transformation and the best yields were observed with the use of a $\mathrm{CH}_{2} \mathrm{Cl}_{2} / \mathrm{H}_{2} \mathrm{O}$ (2:1) biphasic solvent system. Noticeably, formation of the product was not observed when NFSI was used instead of Selectfluor, this observation being in accordance with the notion that Selectfluor is responsible for the oxidation of $\mathrm{Ag}(\mathrm{I})$ catalyst to $\mathrm{Ag}(\mathrm{III})-\mathrm{F}$, a transformation that would be less favorable with the less powerful oxidant NFSI (Scheme 55).

Expanding the above-mentioned methodology, the same team developed the analogous phosphonofluorination of alkenes. ${ }^{[59]}$ In this instance, simple unactivated alkenes 90 were reacted with Selectfluor and catalytic amounts of $\mathrm{AgNO}_{3}$ in the presence of a number of phosphites to give 91. As in the radical aminofluorination, the presence of water was found to be crucial for product formation. While the presence of a base led to complete suppression of the reaction, a mixed solvent system consisting of $\mathrm{CH}_{2} \mathrm{Cl}_{2}: \mathrm{H}_{2} \mathrm{O}: \mathrm{AcOH}(1: 2: 1)$ was found to give the best results. The beneficial effect of such a particular system was rationalized on the basis that a biphasic system prevents the product from further oxidation. Even though the role of the acid remains to be clarified, the authors surmised that stability of $\mathrm{Ag}(\mathrm{III})-\mathrm{F}$ and $\mathrm{Ag}(\mathrm{II})-\mathrm{F}$ is enhanced under acidic conditions. This transforma-

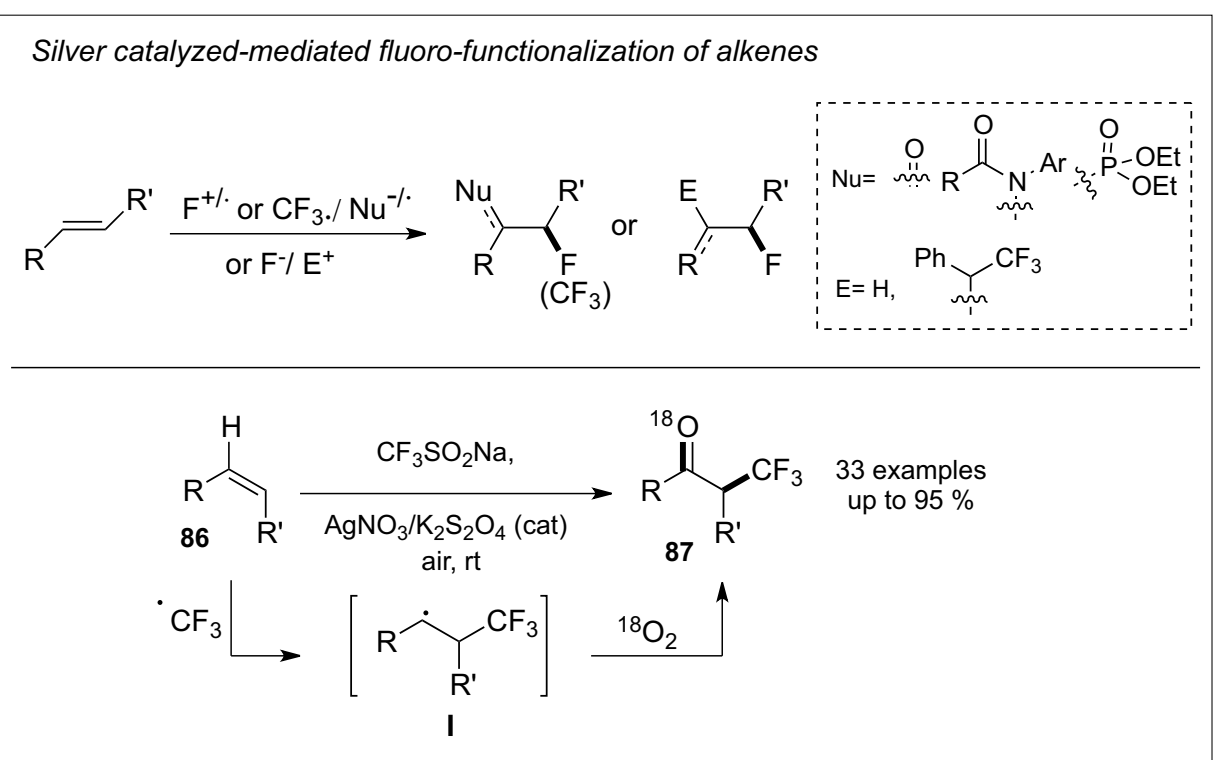

Scheme 54. Oxytrifluoromethylation of alkenes.

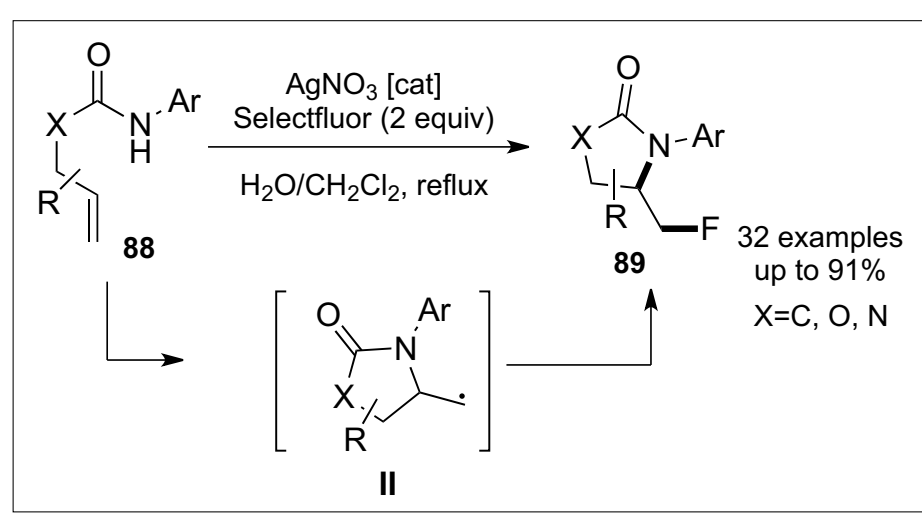

Scheme 55.

Aminofluorination of alkenes.

tion was shown to exhibit a broad substrate scope. Additionally, stereoselective phosphonofluorination of a steroid derivative afforded 91a in $89 \%$ yield with high diastereoselectivity. Nevertheless, electron-poor alkenes and easily oxidized functionalities were not tolerated under the optimized conditions (Scheme 56).

Recent efforts to prepare an elusive $\alpha-\mathrm{CF}_{3}$-benzyl silver species, from the reaction of AgF and gem-difluoroalkenes 92 led to the discovery by $\mathrm{Hu}$ and coworkers, ${ }^{60]}$ of a process for the preparation of symmetric 2,3-bis-(aryl)-1,1,1,4,4,4-hexafluorobutanes 93 that proceeds through a proposed $\mathrm{CF}_{3}$-substituted benzyl radical species IV. These unanticipated results were explained by the thermal instability of in situ generated alkylsilver species, which are prone to dimerize to give alkyl dimers. This is in sharp contrast with the thermal stability exhibited by their perfluorinated counterparts, which are relatively stable at room temperature, thus, capable of engaging in various chemical transformations with other reactive species.[40e] In this transformation, only $\beta, \beta$-difluorostyrene derivatives were shown to homocouple, while other alkyl-substituted gem-difluoroalkenes afforded only the hydrofluorination products. The hexafluorobutanes 93 were generally obtained as a 1:1 mixture of syn/anti diastereoisomers. Notably, the products could also be obtained if a mixture of $\mathrm{CsF}$ and $\mathrm{AgBF}_{4}$ was used instead of $\mathrm{AgF}$ (Scheme 57). Further applications of this novel $\alpha-\mathrm{CF}_{3}$-benzyl silver species remain to be developed and challenges as-

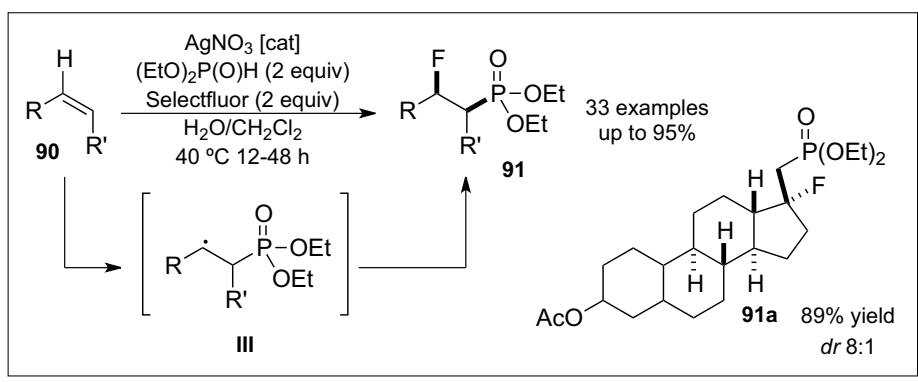

Scheme 56. Phosphonofluorination. 
sociated with its thermal instability still need to be overcome.

In recent years, the reactivity of silver towards carbene and carbenoid species has been recognized as substantially different from other transition metals. ${ }^{[61]}$ For instance, it has been shown that silver vinyl carbenes show electrophilic reactivity at the vinylogous position rather than the carbene site. Exploiting this property and as continuation of their work on silver vinyl carbene species, Davies and coworkers $^{[62]}$ reported a vinylogous fluorination of silver vinyl carbenes to afford $\gamma$-fluoro$\alpha, \beta$-unsaturated esters $\mathbf{9 5}$. In this transformation, the silver carbene species $\mathbf{V}$ was suggested to form in situ from the corresponding vinyl diazoester compounds 94. Nucleophilic fluorination by $\mathrm{Et}_{3} \mathrm{~N} \cdot 3 \mathrm{HF}$ led to the corresponding products in good yields. Using this methodology, the authors were able to prepare a fluorinated derivative of farnesol. Furthermore, in spite of the absence of a chiral ancillary ligand, the fluorinated steroid derivative 95a was successfully synthesized with good diastereoselectivity. The origin of this diastereoselectivity was explained in terms of stereoelectronic effects arising from the conformation adopted by the substrate (Scheme 58).

\subsubsection{Reactions with Arynes}

Arynes are widely recognized as an important class of reactive intermediates in synthetic organic chemistry. ${ }^{[63]}$ Since their discovery, they have found numerous applications; however, it was only recently that they have successfully been used within the context of organofluorine chemistry. In an unprecedented transformation, $\mathrm{Hu}$ and coworkers ${ }^{[64]}$ disclosed a protocol for the silver-mediated vicinal difunctionalization of in situ-generated benzyne intermediates I, obtained from $o$-trimethylsilyl aryl triflates 96. In this fashion, the otherwise difficult to obtain $o$ -

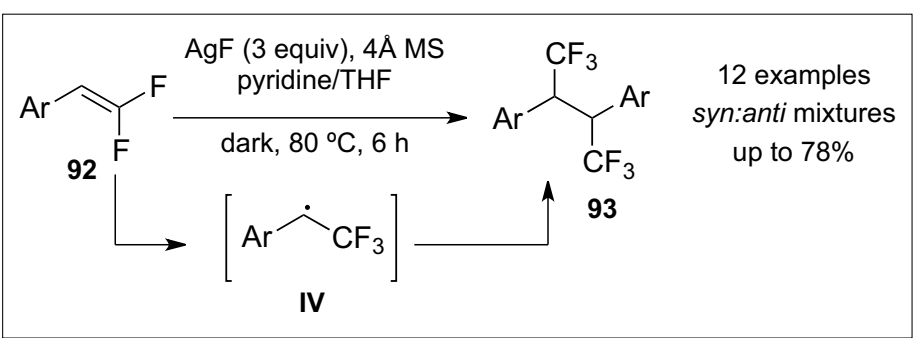

Scheme 57.

Fluorinative homocoupling of gemdifluorostyrenes.

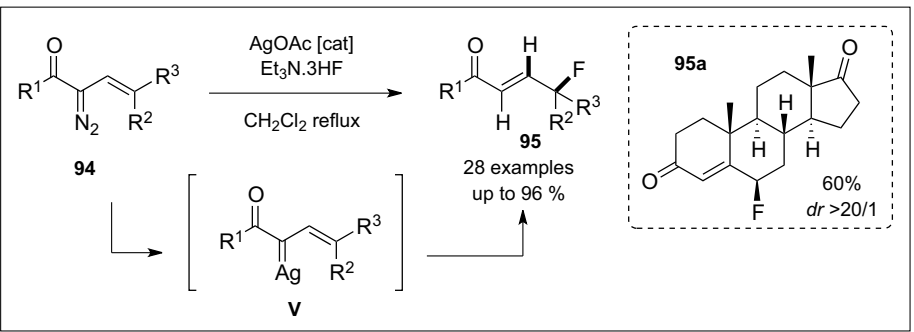

Scheme 58

Vinylogous fluorina-

tion of diazoesters.

iodo-trifluoromethyl arenes 98 were conveniently obtained in good yields with this trifluoromethylation-iodination protocol. The nucleophilic $\mathrm{AgCF}_{3}$ species was generated in situ by the reaction of $\mathrm{TMSCF}_{3}$ and $\mathrm{AgF}$ in a 1:1 ratio. In this transformation, the use of 1-iodophenylacetylene (97) was found crucial for the success of the difunctionalization, while other $\mathrm{X}^{+}$sources such as the $N$-halosuccinimides NCS, NBS and NIS mainly provided the trifluoromethyl arene arising from the protonolysis of the arylsilver species. Likewise, a sterically demanding secondary amine, 2,2,6,6-tetramethyl-piperidine ( $\mathbf{L}$, Scheme 59) was found to be of utmost importance to achieve best results. The role of the ligand was surmised to facilitate the trifluoromethylation step, as well as decreasing the barrier for the second iodination step by the formation of a 6-membered ring transition state (TS) assisted by hydrogen and halogen bonding (Scheme 59).

In a related report, Lee and coworkers, ${ }^{[65]}$ as continuation of their work on aryne chemistry, ${ }^{[66]}$ successfully prepared a series of fluorine-containing hetero- cycles $(\mathbf{1 0 0 a}-\mathbf{c})$. A thermal hexadehydroDiels-Alder reaction of a tetrayne scaffold 99 served as a novel access to benzyne intermediate I, which upon complexation with silver was functionalized by addition of fluoride (from $\mathrm{AgBF}_{4}$ ), $\mathrm{AgCF}_{3}$ (generated in situ from $\mathrm{AgF}$ and $\mathrm{TMSCF}_{3}$ ) and $\mathrm{AgSCF}_{3}$ nucleophiles. As in Hu's method, the authors were able to perform a double functionalization by trapping the arylsilver species with suitable electrophiles such as $N$-halosuccinimides. Although the strict necessity of a fourth alkyne moiety in order to promote the Diels-Alder reaction for aryne formation represents a drawback of this method, the mechanistic novelty and the possibility of assembling a number of biologically relevant fluorinecontaining heterocycles such as indolines, isoindolines and dehydrobenzofurans in a single operational step, overrides such limitation. To illustrate its scope, the method was applied to substrates containing a dihydrocholesterol moiety, thus affording fluorinated, trifluoromethylated and trifluoromethylthiolated derivatives $100 \mathbf{a}-\mathbf{c}$, respectively (Scheme 60).

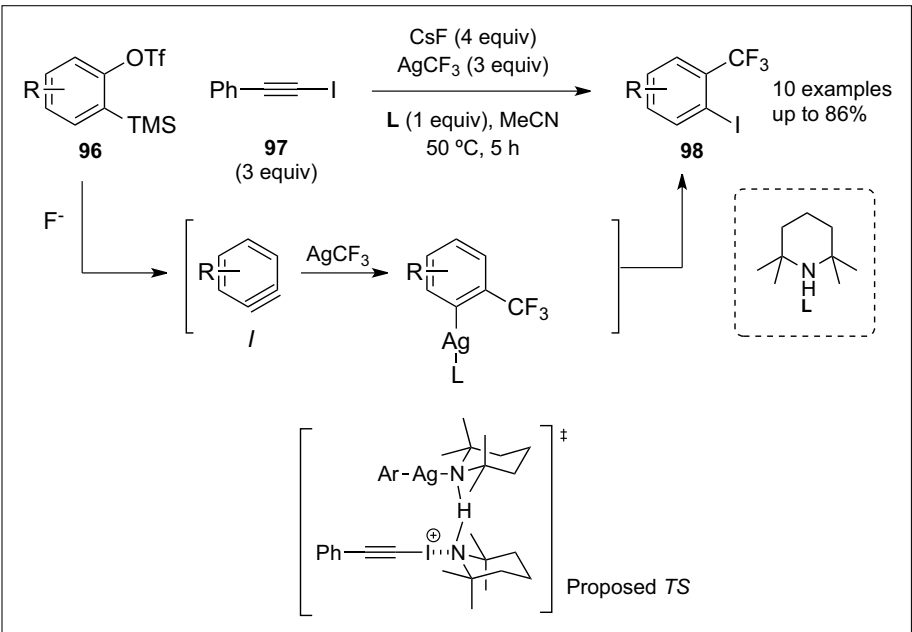

Scheme 59. lodo-trifluoromethylation of arynes.

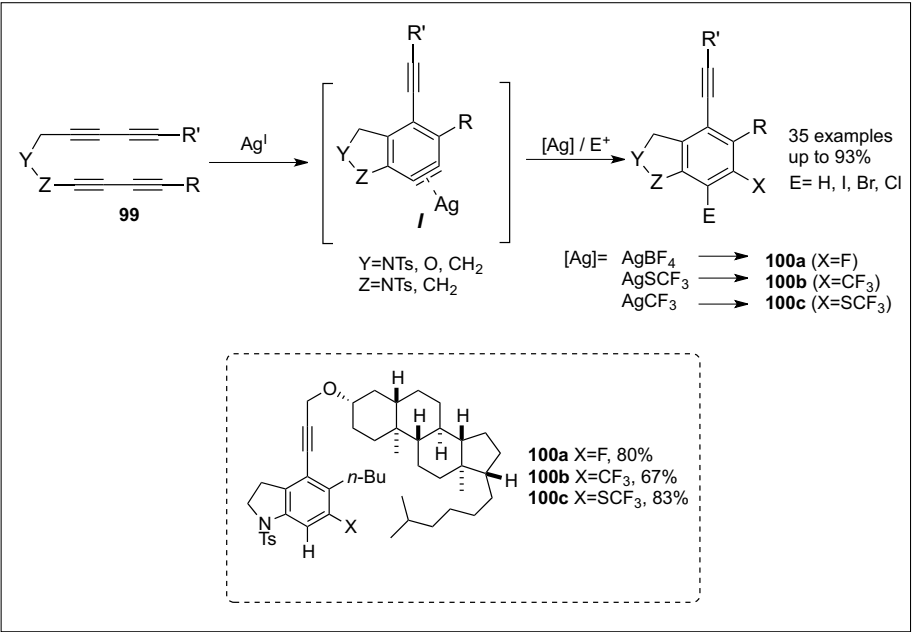

Scheme 60. Fluorine-containing heterocycles. 


\subsubsection{Direct Functionalization with Trifluoromethyl-chalcogen $\left(C F_{3} E\right)$ Fragments}

Due to their special properties, trifluoromethyl ethers, thioethers and selenoethers occupy a privileged position in the agrochemical and pharmaceutical fields. In recent years, the synthesis of $\mathrm{Cs} p^{2}-$ $\mathrm{ECF}_{3}$ compounds $(\mathrm{E}=$ group 6 element $\mathrm{O}$, $\mathrm{S}$ or Se) has received considerable interest. Numerous methodologies ${ }^{[67,68]}$ for the incorporation of $\mathrm{CF}_{3} \mathrm{E}$ moieties based on halogen exchange, radical, electrophilic and nucleophilic approaches have been reported in the literature since the 1930s, however, they often rely on indirect approaches or require hazardous reagents/conditions. On the other hand, more efficient ways to introduce the $\mathrm{CF}_{3} \mathrm{E}$ functionality, namely, the direct cross-coupling of a suitable coupling partner with a pregenerated or in situ formed $\mathrm{CF}_{3} \mathrm{E}$ fragment have long been recognized as a superior strategy, mainly dominated by $\mathrm{Cu}$ - and $\mathrm{Ag}$-mediated transformations. Accordingly, there has been a flurry of activity in the field and new reagents and methods have recently appeared in the chemical literature and previous methodologies have been improved. In this review, such advances will be briefly mentioned; comprehensive reviews are available elsewhere. ${ }^{[68]}$

Recently, a major breakthrough in the synthesis of trifluoromethyl ethers was achieved by Ritter and coworkers. ${ }^{[69]}$ The team was able to perform, for the first time, a direct $\mathrm{C}(\operatorname{aryl})-\mathrm{OCF}_{3}$ bond formation via a silver-mediated oxidative coupling of aryl-stannanes 101a and arylboronic acids 101b with a trifluoromethoxide source TAS-OCF ${ }_{3}(\mathbf{1 0 3})$. As originally reported by Kolomeitsev, ${ }^{[70]}$ in Ritter's report, $\mathbf{1 0 3}$ was conveniently generated in situ from trifluoromethyltriflate $\left(\mathrm{CF}_{3} \mathrm{SO}_{2} \mathrm{OCF}_{3}\right)$ and tris(dimethylamino)-sulfonium difluorotrimethylsilicate (TAS-F). Mediated by stoichiometric amounts of $\mathrm{AgPF}_{6}$ in the presence of F-TEDA-PF ${ }_{6}(\mathbf{1 0 4})$ as an oxidant, a series of aryl nucleophiles $\mathbf{1 0 1}$ afforded aryl trifluoromethyl ethers $\mathbf{1 0 2}$ in good yields (Scheme 61).

It is important to note that, due to the thermal instability of $\mathrm{CF}_{3} \mathrm{O}^{-}$, which decomposes to fluoride and difluorophosgene, to date, similar approaches for the direct construction of $\mathrm{Ar}-\mathrm{OCF}_{3}$ bonds with other transition metals remain unavailable, which is likely to be associated with the usually elevated temperatures that are generally employed to promote such a demanding reductive elimination. Furthermore, catalytic versions of this process are currently unavailable.

In contrast to their oxygen counterparts, direct incorporation of the $\mathrm{SCF}_{3}$ functionality via organometallic species has long been known. Even though $\mathrm{SCF}_{3}$

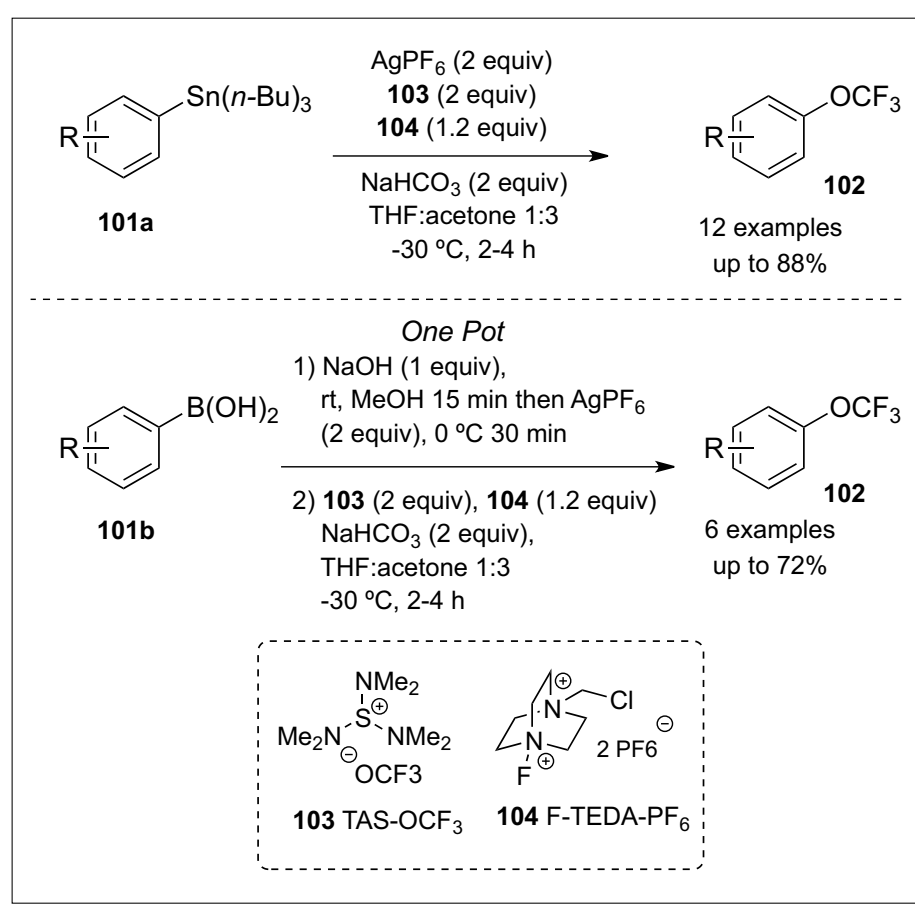

Scheme 61. Silvermediated synthesis of trifluoromethyl ethers from aryl stannanes and boronic acids. chemistry is highly restricted to the copper species $\mathrm{CuSCF}_{3}$ (vide infra), a number of silver-mediated transformations have recently emerged. It is noteworthy that trifluoromethylthiosilver $\left(\mathrm{AgSCF}_{3}\right)$ was first reported by Emeleus ${ }^{[71]}$ in the 1960s, while the more widely used $\mathrm{CuSCF}_{3}$ species was prepared from the former via a metathetical reaction with copper(I) halides by Yagupolskii in 1975.[72]

A recent development in the field of trifluoromethylthiolation chemistry was reported by Wang and coworkers. ${ }^{[73]}$ In their report, a series of oxindoles 106 were prepared by the reaction of alkenes $\mathbf{1 0 5}$ and $\mathrm{AgSCF}_{3}$ as a novel source of $\mathrm{SCF}_{3}$ radicals under oxidative conditions. Noteworthy, radical $\mathrm{SCF}_{3}$ chemistry was largely limited to the gaseous $\mathrm{CF}_{3} \mathrm{SH}, \mathrm{CF}_{3} \mathrm{SCl}$ or $\mathrm{CF}_{3} \mathrm{~S}-\mathrm{SCF}_{3}$ developed 50 years ago.[67g, h] Noticeably, $\mathrm{CuSCF}_{3}$ afforded no product under the optimized conditions, thus, highlighting the role of silver in the generation of ${ }^{-} \mathrm{SCF}_{3}$ radicals (Scheme 62 ).

In a related report, $\mathrm{SCF}_{3}$-containing heterocycles were successfully synthesized starting from $\mathrm{AgSCF}_{3}, o$-alkynyl benzaldoximes 107 and catalytic amounts of AgOTf as a $\pi$-activator. In this fashion, trifluoromethylthiol-substituted isoquinolines 108 were obtained in good yields. The presence of 4-MeO-benzenesulfonyl chloride as activator for the $N$-oxide generated upon addition of $\mathrm{AgSCF}_{3}$ was critical for the success of the process (Scheme 62).

\subsection{Copper-catalyzed Reactions}

Historically, fluoroalkyl and perfluoroalkyl compounds of copper have received more attention than perhaps any other metal. Since the pioneering work by McLoughling and Thrower[74] on the preparation of perfluoroalkylcoppers back in 1969, increasing attention has been paid to this species for the construction of biologically relevant molecules possessing the $\mathrm{CF}_{3}, \mathrm{CF}_{2} \mathrm{H}, \mathrm{SCF}_{3}$ and $\mathrm{SeCF}_{3}$ functionalities and numerous copper-mediated as well as -catalyzed procedures have been reported in the literature. Although older works may be commented where necessary, this work focuses on the developments in the field since 2012. In cases for which some reviews on particular related

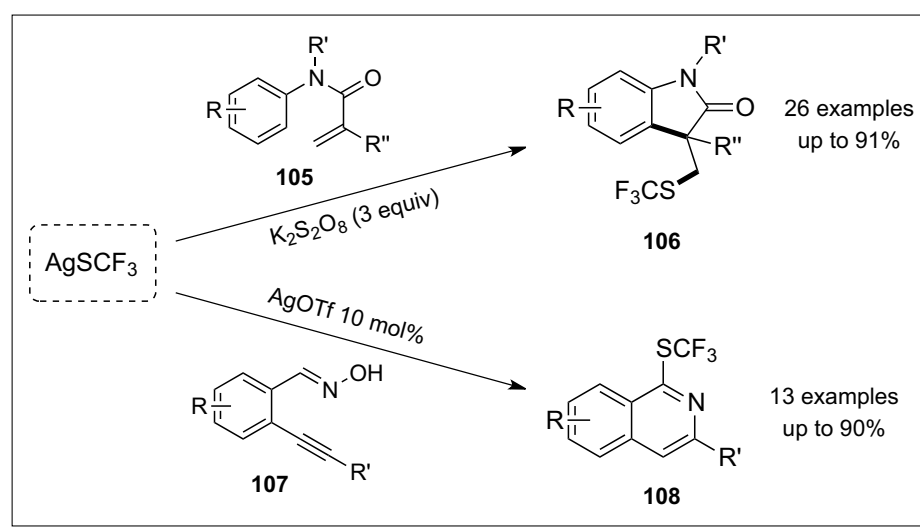

Scheme 62. Synthesis of $\mathrm{SCF}_{3}$ substituted oxindoles and isoquinolines. 
topics have been published more recently, only the literature published thereafter will be considered.

\subsubsection{Aromatic Trifluoromethylation}

Since the pioneering work by Kobayashi and coworkers, ${ }^{[75]}$ who reported the first trifluoromethylation of aryl, vinyl, alkyl, and even heterocyclic halides with in situ generated $\mathrm{CuCF}_{3}$ from $\mathrm{CF}_{3} \mathrm{I}$ and copper powder, a number of other methodologies ${ }^{[76]}$ employing stoichiometric amounts of $\mathrm{Cu}$ were reported. A catalytic transformation with fluorosulfonyl difluoroacetate was developed by Chen ${ }^{[77]}$ and coworkers; however, a major step forward was reported by Amii's group. ${ }^{[78]}$ Their protocol enabled the aromatic trifluoromethylation of (hetero)aryl iodides 109 with the use of $\mathrm{TESCF}_{3} / \mathrm{KF}$ and $10 \mathrm{~mol} \% \mathrm{CuI}$ in the presence of a bidentate chelating ligand 1,10-phenantroline (Scheme 63).

In recent years, there has been a flurry of activity in the field of copper-catalyzed or -mediated aromatic trifluoromethylations and a number of procedures have been developed which rely on an array of common $\mathrm{CF}_{3}$ transfer reagents. Sources of $\mathrm{CF}_{3}$ moiety can be categorized as nucleophilic, electrophilic and radical (Fig. 2).

Important developments in the area include the use of aryl bromides as electrophilic coupling partners, ${ }^{[79]}$ arylboronic acids ${ }^{[79 b, 80]}$ with $\mathrm{CF}_{3}{ }^{+}$sources, as well as with nucleophilic $\mathrm{CF}_{3}$ sources under oxidative conditions. Recently, a rapid synthesis of trifluoromethyl arenes was reported under flow chemistry conditions. ${ }^{[81]}$ Furthermore, room temperature[80f] trifluoromethylations have also been enabled under ligand-free conditions ${ }^{[82]}$ as well as by NHC ligand frameworks ${ }^{[83]}$ (Scheme 64, part $\mathrm{A}$ and $\mathrm{B}$ ).

Until recently, copper-catalyzed or -mediated aromatic trifluoromethylations had relied on aryl halides or arylboronic acids as starting materials for such transformations (Scheme 64, part A and B). However, a major development for the construction of $\mathrm{Ar}-\mathrm{CF}_{3}$ motifs, came independently from the groups of $\mathrm{Fu}^{[84]}$ and Gooßen, ${ }^{[85]}$ when they were able to prepare

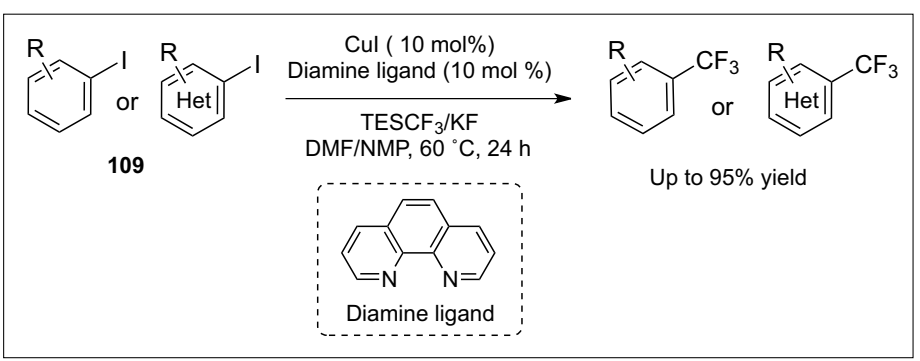

Scheme 63. Aromatic trifluoromethylation catalytic using copper salts.

trifluoromethyl arenes starting from anilines via their aryldiazonium salts prepared in situ or separately (Scheme 64, part C).

$\mathrm{Fu}$ and coworkers utilized an electrophilic $\mathrm{CF}_{3}$ reagent ( $\mathbf{X V}$, Fig. 2) for the synthesis of $\mathrm{CF}_{3}$-substituted arenes. In their report, the aryl diazonium salts were prepared in situ by means of isoamyl nitrite, thus, avoiding the need for purification. These protocols tolerate functional groups such as ether, thioethers, amide, alkynyl and notably, bromide and hydroxyl. Heteroaromatic amines are also suitable substrates for this Sandmeyer trifluoromethylation reaction. Based on mechanistic studies, the authors surmised that this process involves a radical pathway. Thus, a series of trifluoromethylated bicyclic structures was accessed in this fashion. ${ }^{[84]}$

In a similar work, Gooßen's group achieved a copper-mediated trifluoromethylation of a series of aryldiazonium tetrafluoroborates prepared by a diazotization

\section{Common nucleophilic $\mathrm{CF}_{3}$ sources:}

$\mathrm{TMSCF}_{3}(\mathrm{I}), \mathrm{TESCF}_{3}(\mathrm{II}), \mathrm{CF}_{3} \mathrm{COOM}(\mathrm{M}=\mathrm{Na}, \mathrm{K})$ (III), $\mathrm{CF}_{3} \mathrm{H}(\mathrm{IV}), \mathrm{K}\left[\mathrm{CF}_{3} \mathrm{~B}(\mathrm{OMe})_{3}\right](\mathbf{V}), \mathrm{FSO}_{2} \mathrm{CF}_{2} \mathrm{CO}_{2} \mathrm{Me}(\mathrm{VI})$

$$
\text { (VII) }
$$

Common electrophilic or radical $\mathrm{CF}_{3}$ sources:

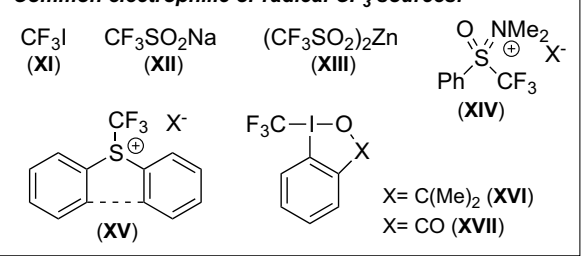

Fig. 2. Sources of $\mathrm{CF}_{3}$ functionality. reaction of the corresponding anilines. The isolated salts were then reacted with $\mathrm{CuCF}_{3}$ formed in situ from the Ruppert-Prakash reagent (I) and $\mathrm{CuSCN}$ to obtain $\mathrm{Ar}-\mathrm{CF}_{3}$ motifs in good yields. This transformation tolerated a range of electron-poor and electron-rich functional groups such as keto, cyano, ester and amino. Remarkably, iodo substituents were not affected under the reaction conditions, thus dramatically expanding the possibilities for further functionalization. ${ }^{[85]}$

Of particular significance is the successful utilization of fluoroform $\left(\mathrm{CF}_{3} \mathrm{H}\right)$ as a direct source of the $\mathrm{CF}_{3}$ motif. The importance is centered on the fact that fluoroform (a by-product of Teflon ${ }^{\circledR}$ manufacturing) is produced in amounts exceeding 20,000 tons annually. Furthermore, it is a potent green house gas with an estimated global warming potential 11,700 times greater than that of $\mathrm{CO}_{2} \cdot{ }^{[86]}$ Thus, its efficient utilization for the synthesis of valuable chemicals and building blocks is of remarkable importance. Accordingly, the groups of Grushin ${ }^{[87]}$ and Prakash ${ }^{[88]}$ independently developed conditions for its direct utilization in several important chemical transformations (Scheme 65).

The direct cupration of fluoroform (IV) was first reported by Grushin and coworkers by means of a novel dialkoxycuprate 110, obtained through the reaction of $\mathrm{CuCl}$ and 2 equiv of $t$-BuOK in DMF (Scheme $65)$. This process enabled the copper-mediated trifluoromethylation of arylboronic acids, ${ }^{[87 b]}$ aryl iodides, aryl bromides and even some aryl chlorides. ${ }^{[87 d]}$ In Grushin's report, arylboronic acids were smoothly trifluoromethylated at room temperature employing 2 equiv of fluoroform-derived

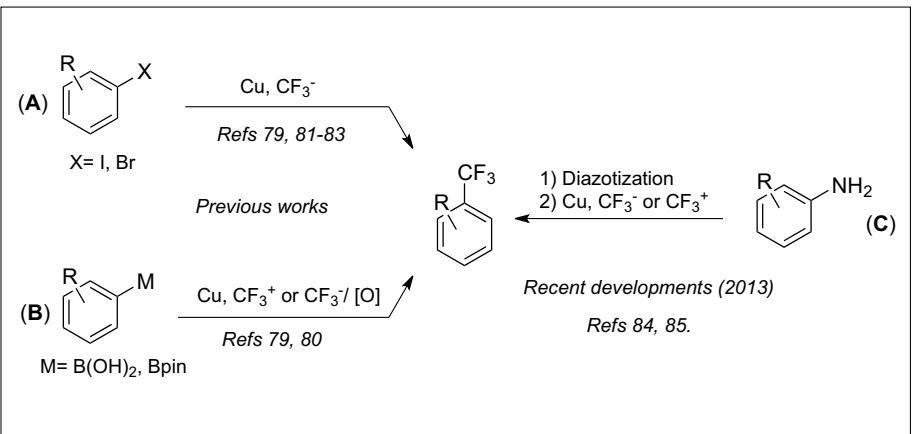

Scheme 64. Aromatic trifluoromethylation of aryl halides, arylboron species and anilines.

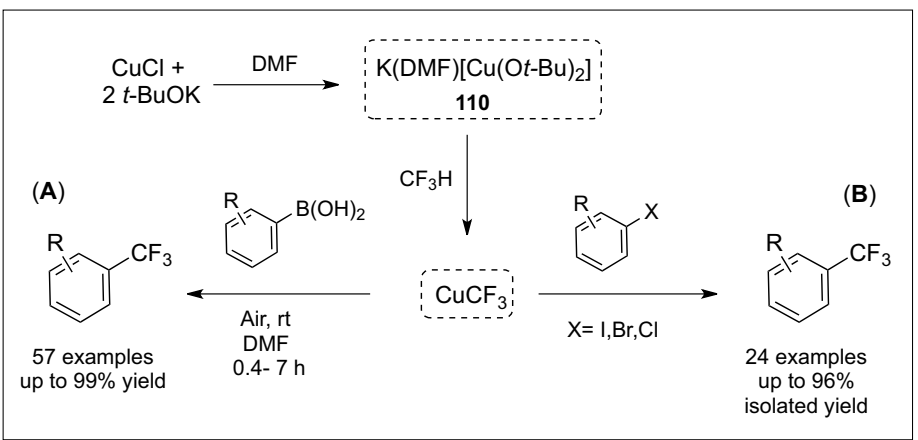

Scheme 65. Trifluoromethylation of aromatics by fluoroform-derived $\mathrm{CuCF}_{3}$. 
$\mathrm{CuCF}_{3}$. In some cases, reactions were performed at $0{ }^{\circ} \mathrm{C}$ in order to minimize the proto-deborylation by-product. This method is particularly attractive due to the low cost of $\mathrm{CF}_{3} \mathrm{H}$ and the use of air as the oxidant. Furthermore, no ligand or external additive was necessary to promote the transformation. Arguably, the cost-effectiveness of this transformation overcomes the issue of using super-stoichiometric quantities of $\mathrm{Cu}$ (Scheme 65, part A).

For the case of aryl halides, most aryl and heteroaryl iodides were smoothly trifluoromethylated at temperatures from 25 ${ }^{\circ} \mathrm{C}$ to $60^{\circ} \mathrm{C}$ with 1.2 to 2.0 equiv of $\mathrm{CuCF}_{3}$. On the other hand, only electron-poor aryl bromides and 2-bromopyridines afforded the corresponding products in practical yields at elevated temperature $\left(c a .60^{\circ} \mathrm{C}\right)$ (Scheme 65, part B). The isomeric 3-bromopyridines are much less reactive and can only be obtained in yields of up to $17 \%$. In this transformation, an interesting ortho effect was observed, i.e. substrates with electron-withdrawing substituents in the ortho position were particularly reactive, thus, providing high yielding products. As expected only highly electrophilic heteroaryl chlorides with ortho electron-withdrawing moieties were reactive enough to afford the corresponding products, albeit in lower ${ }^{19} \mathrm{~F}$ NMR yields and requiring higher reaction temperature $\left(80^{\circ} \mathrm{C}\right)$.

\subsubsection{Aromatic Difluoromethylation}

Amongst fluoroalkyl moieties, the difluoromethyl $\left(\mathrm{CF}_{2} \mathrm{H}\right)$ group has attracted increasing attention due to its potential application in the pharmaceutical arena. The difluoromethyl group is bioisosteric with the carbinol $(\mathrm{OH})$ group, yet reasonably lipophilic. Additionally, it can act as a hydrogen bond donor. For these properties, the $\mathrm{CF}_{2} \mathrm{H}$ motif has been used in the preparation of numerous biologically active compounds such as sugars, ${ }^{\left[{ }^{89]}\right.}$ enzyme inhibitors and agrochemicals. ${ }^{[90]}$ In sharp contrast to its perfluoro counterpart, direct incorporation of this functionality is extremely rare. Most of the methods available rely on indirect methods such as deoxofluorination of aromatic aldehydes.

Recently, a multistep procedure involving a copper-catalyzed cross-coupling of aryl iodides with $\mathrm{TMSCF}_{2} \mathrm{CO}_{2} \mathrm{Et}$, hydrolysis and decarboxylation sequence was reported by Amii's group. ${ }^{[91]}$ In this sequence, the last decarboxylation step was successful only for electron-poor substrates, thus limiting the reaction scope (Scheme 66, part A). To date, there are only two reports on the direct and regiospecific difluoromethylation of aromatics; both relying on copper-mediated transformations.

Fier and Hartwig[92] disclosed a method for the direct difluoromethylation of aryl and vinyl iodides with 5 equiv of a difluo- romethyl analog of the Ruppert-Prakash reagent $\mathrm{TMSCF}_{2} \mathrm{H}$ and stoichiometric amounts of CuI. In this transformation electron-rich and electron-neutral iodoarenes are suitable substrates, however, electron-withdrawing functionalities afforded only the protodehalogenated products and a competitive difluoromethylation of carbonyl moieties was observed (Scheme 66, part B).

In a related report, Prakash and coworkers ${ }^{[93]}$ introduced a novel reagent for the direct incorporation of the $\mathrm{CF}_{2} \mathrm{H}$ functionality, namely, tributyl(difluoromethyl) stannane $n$ - $\mathrm{Bu}_{3} \mathrm{SnCF}_{2} \mathrm{H}$. This non-volatile reagent was conveniently accessed from the reaction of $\mathrm{TMSCF}_{3}$ and $n-\mathrm{Bu}_{3} \mathrm{Sn}$ $\mathrm{H}$ via difluorocarbene insertion ${ }^{[94]}$ into the $\mathrm{Sn}-\mathrm{H}$ bond. In this fashion, difluoromethylations of aryl and heteroaryl iodides proceed smoothly with 2-3 equiv of $n-\mathrm{Bu}_{3} \mathrm{SnCF}_{2} \mathrm{H}$ in DMA at $100-120^{\circ} \mathrm{C}$. Contrary to Hartwig's protocol, electronwithdrawing groups are tolerated affording the corresponding difluoromethyl arenes in good yields. Remarkably, owing to the reduced oxophilicity of tin $v s$ silicon, aldehyde, keto and even ester moieties are unaffected, thus, carbonyl difluoromethylation was not observed (Scheme 66, part C).

\subsubsection{Ar-F Bond Formation}

The generation of C(aryl)-F bonds catalyzed by copper was recently reported by Ribas and coworkers (Scheme 67). ${ }^{[95]}$ A macrocyclic arylcopper(III) complex 112 generated after oxidative addition of an aryl halide $\mathbf{1 1 1}$ to a $\mathrm{Cu}$ (I) catalyst, was shown for the first time, to undergo facile $\mathrm{C}-\mathrm{F}$ reductive elimination at room temperature in acetonitrile, a transformation previously limited to Pd species. Noteworthy, the same protocol could be used to perform the halogen exchange in any direction with the use of appropriate halide salts. Remarkably, even defluorination was possible at room temperature. These outstanding results however, are limited to the very specific macrocyclic ligand framework and their application to more general substrates was not reported. Nevertheless, Ribas' seminal work paved the way for the discovery and development of more general $\mathrm{Cu}$ mediated fluorination reactions.

Soon after Ribas' report, Hartwig and coworkers disclosed a $\mathrm{Cu}$-mediated protocol for the fluorination of aryl iodides using $\mathrm{AgF}$ as the fluoride source. As noted by previous authors, the success in the transformation relies heavily on weakly coordinating counterions and ligands on $\mathrm{Cu}$, thus, after screening a series of nitrileligated copper(I) species, it was found that the best results were obtained with the use of 3 equiv of $(t \text {-BuCN })_{2}$ CuOTf in DMF at $140{ }^{\circ} \mathrm{C}$. In this manner, electron-poor as well as electron-rich iodoarenes were smoothly converted into the corresponding aryl fluorides (Scheme 68, part A).

Next, the same group reported an alternative approach starting from arylboronate esters and the electrophilic fluoride reagent 114. In this transformation, the choice of the base proved to be of critical importance. Not only transmetallation must be promoted, but also base-induced decomposition of the $\mathrm{F}^{+}$source must be avoided. To that end, only AgF successfully afforded the corresponding products in good yields, whereas other alkoxide bases were less effective. The authors surmised that the success of $\mathrm{AgF}$ as a base was due to its lower solubility and nucleophilicity, thus, preventing an unproductive transmetallation of the arylboronate to a $\mathrm{Cu}(\mathrm{I})$ species. Furthermore, ${ }^{19} \mathrm{~F}$ NMR spectroscopy studies, made the identification of an $\mathrm{Cu}(\mathrm{III})$-fluoride species, analogous to the
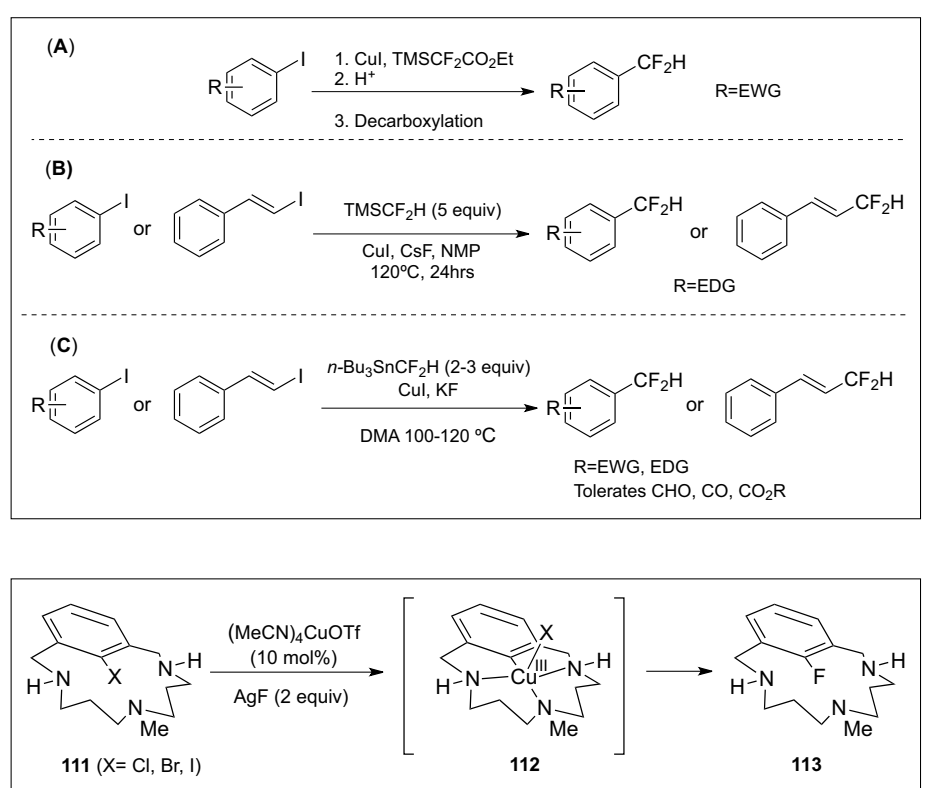

Scheme 66.

Difluoromethyl arenes by three-step sequence $(A)$, direct cross-coupling with $\mathrm{TMSCF}_{2} \mathrm{H}(\mathrm{B})$ and from $n-\mathrm{Bu}_{3} \mathrm{SnCF}_{2} \mathrm{H}$ (C).

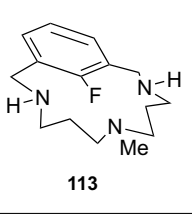

Scheme 67. First copper-catalyzed C-F bond formation. 


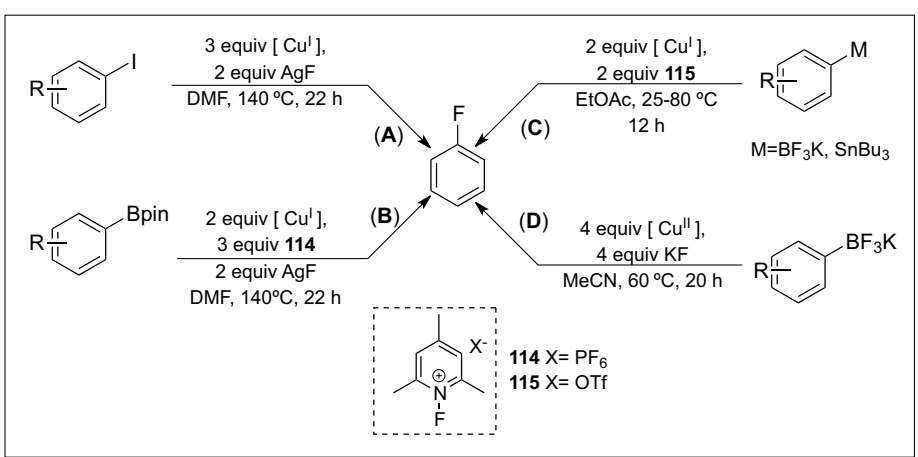

Scheme 68. Coppermediated aromatic fluorination of aryl iodides (A), arylboron pinacolates $(B)$, aryltrifluoroborates $(\mathrm{C})$, stannanes and oxidative fluorination of aryltrifluoroborates (D).

one described by Ribas (vide supra), possible. Subsequent rate-limiting transmetalation afforded an arylcopper(III) fluoride which underwent rapid reductive elimination (Scheme 68, part B).

A very similar approach is the utilization of aryl trifluoroborates and aryl stannanes as nucleophilic coupling partners. As outlined by Sandford, ${ }^{[96]}$ such arylboron and aryltin species can be fluorinated with the use of $\mathbf{1 1 5}$ as an electrophilic fluorine source and 2 equiv of $(t \text {-BuCN })_{2}$ CuOTf, under mild conditions. Interestingly, and contrasting with Hartwig's report, arylboron pinacolates afforded only traces of the desired product under these conditions. In the case of aryl stannanes, fluorinations occurred at room temperature, while aryltrifluoroborates required heating at $80^{\circ} \mathrm{C}$. Both electron-poor and electron-rich substrates were suitable substrates for this transformation, achieving the corresponding products in practical yields (Scheme 68, part C).

A considerable improvement was later disclosed by Sanford's group, ${ }^{[97]}$ who published a procedure for the fluorination of aryltrifluoroborates from the inexpensive $\mathrm{KF}$ mediated by $\mathrm{Cu}(\mathrm{OTf})_{2}$. The use of an inexpensive fluorine source, as well as the operational simplicity and mild reaction conditions, make this method particularly attractive for large-scale industrial applications. The role of $\mathrm{Cu}(\mathrm{OTf})_{2}$ is believed to be twofold: as the promoter for the $\mathrm{C}-\mathrm{F}$ bond formation and as an oxidant. This hypothesis is in agreement with the observation that $>2$ equiv of $\mathrm{Cu}(\mathrm{OTf})_{2}$ are required in order to achieve good yields. In this fashion, substrates bearing electron-donor, electron-withdrawing, as well as carbonyl groups is successfully fluorinated. Heteroaromatic substrates are also fluorinated, albeit in a modest yield (20\%). As in previous reports, a high valent $\mathrm{Cu}$ (III) intermediate is surmised to be responsible for the $\mathrm{C}-\mathrm{F}$ bond formation event (Scheme 68, part D).

In spite of such developments, all these methods for the construction of C(aryl)-F bonds suffer several drawbacks that are mainly associated with the contamination by side products and product purifica- tion. In all of the above-mentioned reports (Scheme 68), products are often contaminated by the virtually inseparable arenes, originated from the protodehalogenation or protodeborylation side reactions. Furthermore, catalytic versions of such transformations are currently unavailable, the only examples being possible via $\mathrm{Ag}$ or Pd catalysis (vide supra).

\subsubsection{Trifluoromethylation of Olefinic C-H Bonds}

In recent years, a number of coppercatalyzed procedures that enable the direct trifluoromethylation of $\mathrm{C}-\mathrm{H}$ bonds have been published. These protocols include: a) methods in which a $\mathrm{Csp}^{2}-\mathrm{CF}_{3}$ motif can be accessed and b) methods for the construction of $\mathrm{Csp}^{3}-\mathrm{CF}_{3}$ motifs via olefin difunctionalization reactions (Scheme 69). The most recent protocols are commented on below.

\subsubsection{Construction of $\mathrm{Csp}^{2}-\mathrm{CF}_{3}$ Bonds}

Recently, the groups of Szabo ${ }^{[98]}$ and Wang ${ }^{[99]}$ disclosed a protocol for the direct $\mathrm{C}-\mathrm{H}$ trifluoromethylation of quinones $\mathbf{1 1 6}$. In Wang's report, the transformation was performed with catalytic amounts of $\mathrm{CuI}$ (ca. $20 \mathrm{~mol} \%$ ). Owing to the dichotomous nature of the hypervalent iodine reagent

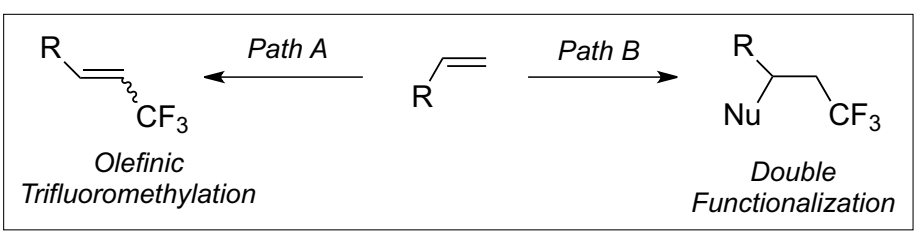

Scheme 69

Pathways for olefin trifluoromethylation.

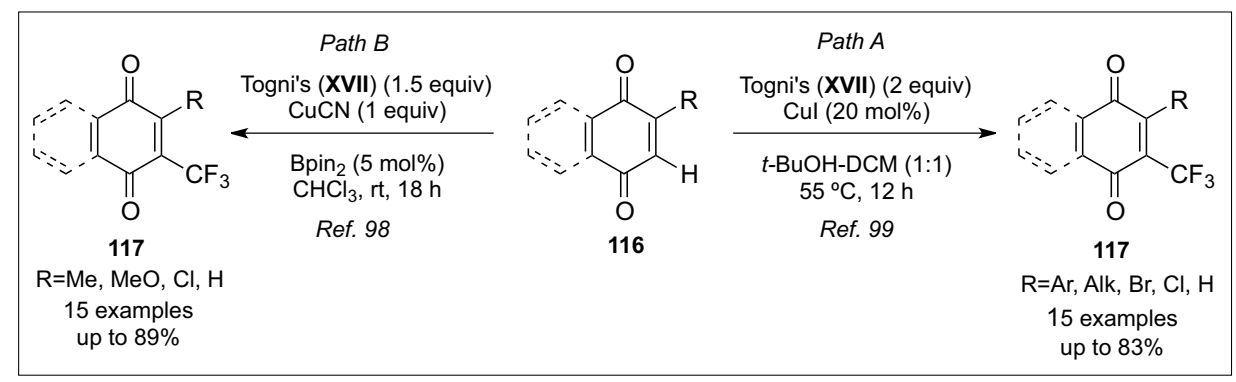

Scheme 70. Direct quinone $\mathrm{C}-\mathrm{H}$ trifluoromethylation.

rifluoromethylation Functionalization up to $83 \%$
XVII (Fig. 2), ${ }^{\circ} \mathrm{CF}_{3}$ radicals can be generated in situ after an initial SET from the $\mathrm{Cu}$ (I) catalyst. The ensuing $\mathrm{CF}_{3}$ addition to the olefinic double bond and subsequent deprotonation afforded the corresponding products in good yields. In this fashion, quinones and naphtoquinones possessing either electron-rich or -deficient aryl substituents 117 were successfully prepared. Notably, halogen substituents were unaffected under the reaction conditions, thus, enabling further chemical transformations (Scheme 70, path A).

Alternatively, Szabo's procedure employed 1.5 equiv of XVII, stoichiometric amounts of a $\mathrm{Cu}(\mathrm{I})$ salt as well as catalytic amounts of bis(pinacolato)diboron (5 mol\%) as a radical activator. Nevertheless, contrasting with Wang's procedure, the stoichiometric version of this transformation enabled the bis(trifluoromethylation) of substrates by employing 2 equiv of Togni's reagent. In this fashion, a series of $\mathrm{CF}_{3}$-functionalized quinones and naphtoquinones bearing $\mathrm{Me}, \mathrm{MeO}$ and chloro substituents were prepared in good yields (Scheme 70, path B).

Copper catalysis has enabled olefinic trifluoromethylations of a series of enamides at room temperature to obtain 119. As outlined by Feng and Loh,[100] $N$-vinyl acetamides 118 were reacted with 1.1 equiv of Togni's reagent XVII in THF at room temperature in the presence of $10 \mathrm{~mol} \%$ of the cationic copper species $(\mathrm{MeCN})_{4} \mathrm{CuPF}_{6}$. Interestingly, single crystal X-ray diffraction analysis of the product revealed a trans configuration with respect to the nitrogen atom. In this transformation, functionalities such as acetyl, cyano, sulfonyl and chloro were tolerated, thus enabling further functionalizations. A single heterocyclic enamide was also a suitable substrate albeit affording the desired product in only $50 \%$ yield even with a higher catalyst loading ( $c a .20 \mathrm{~mol} \%$ ) (Scheme 71). 
Building upon this transformation, the same team reported a procedure for an olefinic $\mathrm{C}-\mathrm{H}$ trifluoromethylation of alkenes.[101] This time, by employing a series of $N$-tosyl-protected acrylamides 120, 1.2 equiv of Togni's reagent and 10 $\mathrm{mol} \% \mathrm{CuCl}$, the authors were able to obtain $\beta$-trifluoromethyl enamides $\mathbf{1 2 1}$ in good yields and with a $Z$-configuration exclusively. Importantly, the success of this transformation greatly relies on the presence of the $N$-tosyl group, since other directing groups such as NHBn, NHPh, $\mathrm{OH}$ and $\mathrm{OMe}$ all failed to afford the desired product. The authors surmised that the increased acidity of the NH moiety was likely to be associated with that observation. In this manner, electron-donating as well as electron-withdrawing $\alpha$-aryl acrylamides were smoothly converted into products. Notably, the thioether moiety was compatible with this reaction and no catalyst poisoning was observed in that case. Furthermore, $\alpha$-alkyl acrylamides were also suitable substrates. In that case, the products were obtained as mixtures of allylic- $\mathrm{CF}_{3}$ and the desired olefinic- $\mathrm{CF}_{3}$ products; nevertheless, the desired product was the major one in all cases (Scheme 72). A short time later, a very similar protocol employing Umemoto's reagent $\mathbf{X V}$ and $N, N$-diethyl acrylamides was also reported. ${ }^{[102]}$

Very recently, Bi and coworkers ${ }^{[103]}$ disclosed a synthetic protocol for the $\alpha$-trifluoromethylation of $\alpha, \beta$-unsaturated carbonyl compounds. In their report, the olefinic trifluoromethylation was possible through the reaction of 1.5 equiv of Togni's reagent and a series of enones, enamides as well as $\alpha, \beta$-unsaturated esters, thioesters and amides $\mathbf{1 2 2}$ in the presence of $10 \mathrm{~mol} \% \mathrm{CuI}$. Mechanistic investigations suggest that a ${ }^{\circ} \mathrm{CF}_{3}$ radical generated by a SET from CuI is likely to be involved. In this process, the products $\mathbf{1 2 3}$ were regioselectively trifluoromethylated at the $\alpha$-position. Furthermore, the geometry around the double bond was determined to be of an $E$ configuration. Although the authors did not comment on the origin of the regio- and stereoselectivity, this nonetheless, certainly represents an important synthetic procedure that complements the one previously described by Loh (vide supra). To further illustrate the synthetic applicability of their procedure, the team successfully prepared a series of biologically active trifluoromethylated compounds including the antiviral agent trifluridine with a $76 \%$ yield (Scheme 73).

An alternative approach for the preparation of $\alpha$-trifluoromethyl carbonyl compounds was reported by Yu's group. ${ }^{[104]}$ In this process, the authors were able to perform a copper-catalyzed trifluoromethylation reaction of a series of cyclic and

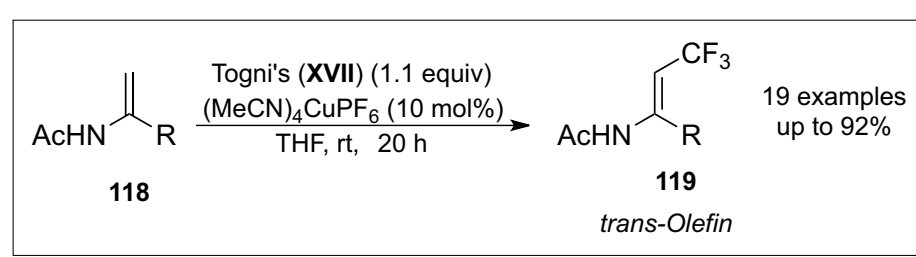

Scheme 71. Room

temperature preparation of $(E)$ $\beta$-trifluoromethyl enamides.

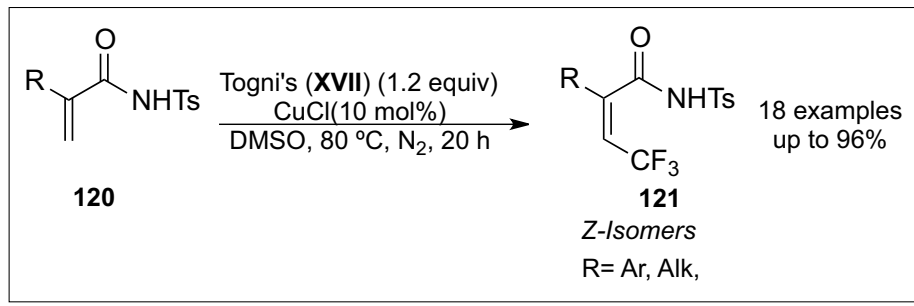

Scheme 72.

Synthesis of $(Z)-\alpha, \beta-$ unsaturated trifluoromethyl amides.

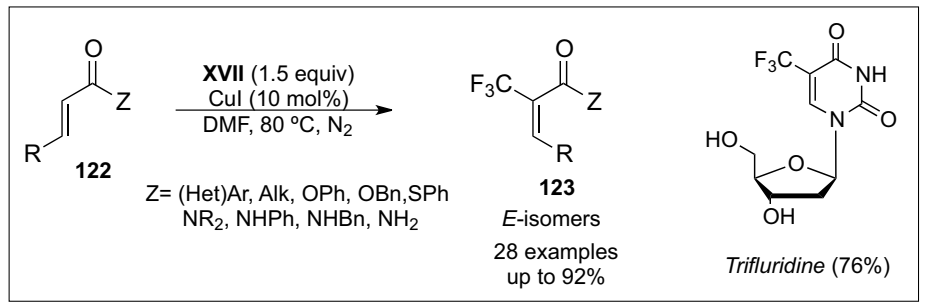

Scheme 73.

Synthesis of $\alpha$-trifluoromethyl $\alpha, \beta$ unsaturated carbonyl compounds.

acyclic $\alpha$-oxoketene dithioacetals 124 with the Ruppert-Prakash reagent under oxidative conditions in the presence of $10-20 \mathrm{~mol} \%$ of a $\mathrm{Cu}$ (II) salt. Addition of the bidentate ligand 1,10-phenantroline, 3 equiv of $\mathrm{KF}$ as a base and 2 equiv $\mathrm{Ag}_{2} \mathrm{CO}_{3}$ as an external oxidant was found to be critical for achieving good yields, while oxidants such as $\mathrm{PhI}(\mathrm{OAc})_{2}$ or ligand-free conditions failed to provide the desired products $\mathbf{1 2 5}$. In this transformation, benzoyl substrates bearing methyl, methoxy, chloro and fluoro substituents were tolerated. Additionally, cinnamoyl ketene dithioacetals were also trifluoromethylated, albeit in a substantially lower yield. Notably, heteroaroyl (furoyl and thienoyl) as well as an ester ketene dithioacetal were found to be suitable substrates, affording the corresponding products in good yields. Furthermore, not only dithioacetals 124, but also monomethylthio acetals $\mathbf{1 2 6}$ were found to be suitable substrates. However, the latter afforded products as a mixture of $E / Z$ isomers. In that work, one example of trifluoromethylation of an internal olefin was reported in $68 \%$ yield (127). Although an isolated case, this result clearly demonstrates the possibility to circumvent the limitations involved with the incorporation of dithioacetal activating groups. To further illustrate the synthetic applicability of the method, the authors prepared a series of trifluoromethyl-pyrimidines $\mathbf{1 2 9}$ via a condensation of $\mathbf{1 2 8}$ with guanidine (Scheme 74).

\subsubsection{Alkene Double Functionalization: Construction of $\mathrm{Csp}^{3}-\mathrm{CF}_{3}$ Bonds}

As previously mentioned, electrophilic $\mathrm{CF}_{3}$ sources can also enable the double functionalization of olefins if coupled with external nucleophiles. Alternatively, the nucleophilic fragment can arise from the

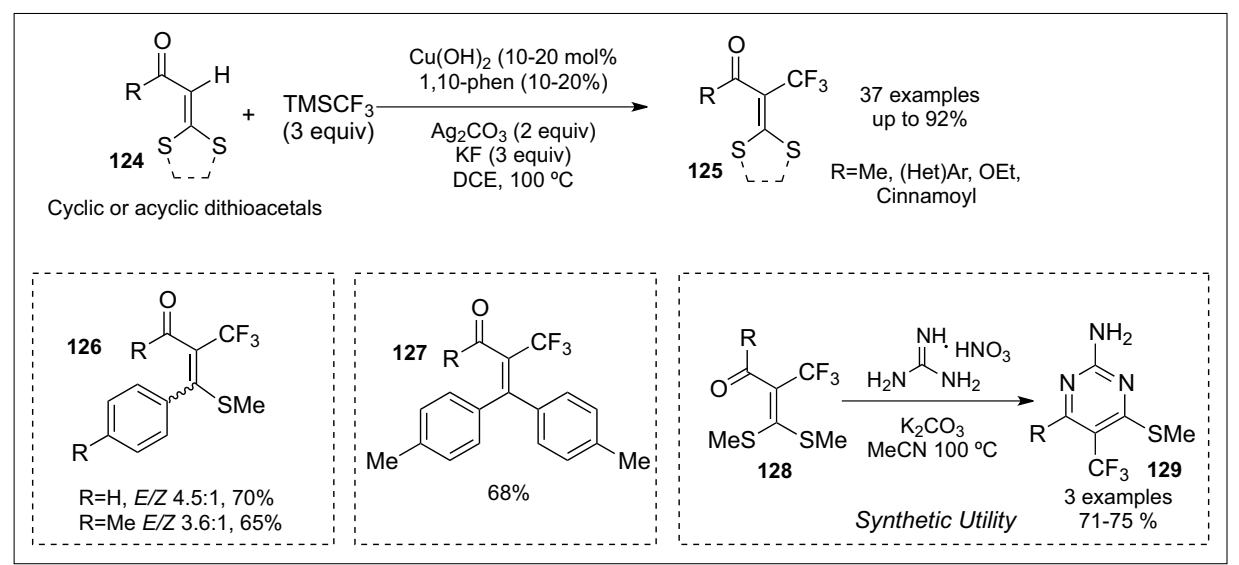

Scheme 74. Trifluoromethylation of $\alpha$-oxoketene dithioacetals. 
same electrophilic reagent, as in the case of the carboxylate fragment from Togni's reagent (vide infra).

Along these lines, Szabo[105] and Sodeoka ${ }^{[106]}$ independently disclosed a method for the trifluoromethyl-benzoyloxylation of alkenes by means of Togni's reagent XVII and olefin derivatives 130, in the presence of catalytic or stoichiometric $\mathrm{Cu}(\mathrm{I})$ salts. In this fashion, a series of styrene derivatives were trifluoromethylated at the $\beta$ position with subsequent addition of the carboxylate moiety at the $\alpha$ position of 132. Intriguingly, when performed in the presence of stoichiometric amounts of $\mathrm{CuX}(\mathrm{X}=\mathrm{I}, \mathrm{Br})$ and vinyl silanes or sulfides 131 as substrates, an $\alpha$-addition of halide ion was observed instead of the expected carboxylate fragment 133. Although these methods are limited to the incorporation of halide or carboxylate as nucleophiles, they certainly showcase the possibilities of $\mathrm{Cu}$ catalyzed/mediated alkene double functionalization chemistry and in some ways, complement the silver chemistry reported in this area (vide supra) (Scheme 75).

Recently, a significant improvement in terms of the scope of O-nucleophiles that can be employed in oxytrifluoromethylation reactions was disclosed by Buchwald's group. ${ }^{[107]}$ In their report, the team was able to employ a series of nucleophiles other than the 2-iodo-phenyl carboxylate fragment using Togni's reagent. In this fashion, upon addition of the $\mathrm{CF}_{3}$ motif from the electrophilic Togni's reagent, simple carboxylates, primary alcohols, phenols and even allylic alcohols were able to trap the thus-generated intermediate in an intramolecular fashion. Consequently, by varying the chain size, a series of trifluoromethyl substituted lactones and various heterocycles were accessed (Scheme 76). Remarkably, enantiopure allylic alcohols gave rise to the corresponding trifluoromethyl epoxides without a drop in enantiomeric excess. Keys to the success of this protocol include the use of the cationic copper complex (MeCN) ${ }_{4} \mathrm{CuPF}_{6}$ as well as a quinoline-based bidentate ligand L1 (Scheme 76).

A similar approach for the oxytrifluoromethylation of enamides $\mathbf{1 3 5}$ was reported by Loh. ${ }^{[100]}$ In that work, quantitative yields of a series of trifluoromethyl aminals 136 were obtained simply by the reaction of a series of enamides with Togni II reagent (XVII) in the presence of $10 \mathrm{~mol} \% \mathrm{CuCl}$ in methanol at room temperature (Scheme 77). In this fashion, substrates possessing electron-withdrawing as well as electronreleasing substituents in the aromatic ring were smoothly transformed. Furthermore, substrates bearing heteroaromatic substituents were also efficiently transformed into products in excellent yields. It must be further emphasized that this transforma-

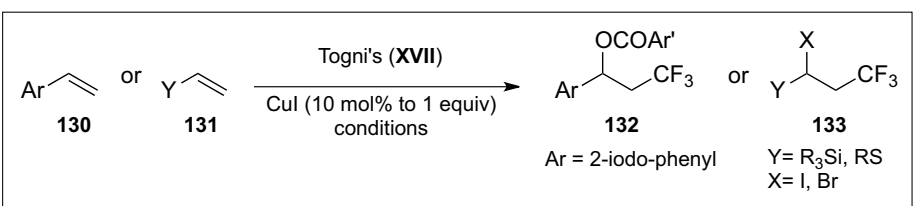

Scheme 75

Trifluoromethylbenzoyloxylation and trifluoromethyl-halogenation of alkenes.

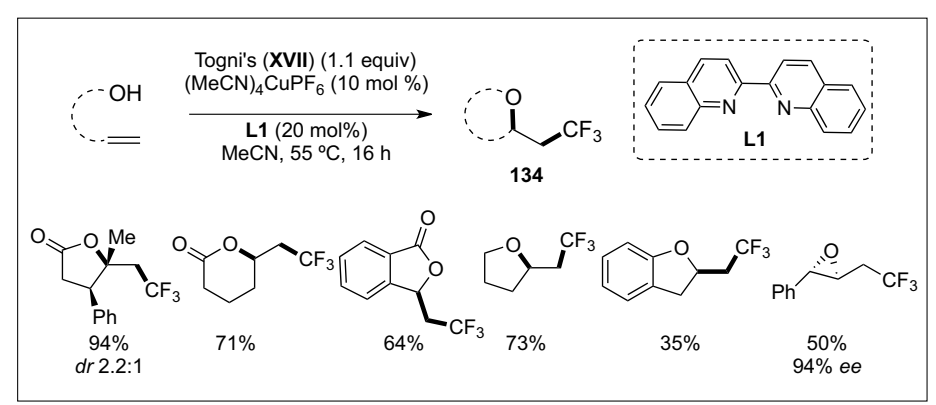

Scheme 76

Oxytrifluoro-

methylation of unactivated alkenes.

tion was shown to be very clean, thus products were obtained in pure form simply by a $\mathrm{NaHCO}_{3}$ wash, thus avoiding column chromatography (Scheme 77).

Very recently, Qing[108] reported a variant of the above mentioned reaction. In that work, the team was able to utilize an alternative $\mathrm{CF}_{3}$ source, namely, the Langlois reagent $\mathrm{NaSO}_{2} \mathrm{CF}_{3}$ as a source of ${ }^{\circ} \mathrm{CF}_{3}$ radicals. Additionally, with the use of $\mathrm{N}$-hydroxy-carbamate $\mathbf{1 3 7}$ as the O-nucleophile, they were able to perform a copper-catalyzed oxytrifluoromethylation of alkenes in a three-component fashion. Styrene derivatives bearing electron-donating and electron-withdrawing groups were suitable substrates. Noticeably, even unactivated alkenes were shown to undergo double functionalization under the reaction conditions. Furthermore, $\alpha, \beta$ unsaturated esters, ketones and amides were also efficiently transformed. In order to illustrate the scalability of the process, the authors prepared $1.2 \mathrm{~g}$ of substrate 138a in $71 \%$ yield. In addition, this product was efficiently reduced to the corresponding $\beta$-trifluoromethyl alcohol in $90 \%$ yield.
However, it is noteworthy that a current limitation of this protocol is that an excess of alkene was necessary in order to achieve good yields (Scheme 78).

Building upon the above methodologies, efforts have focused on the development of double functionalization of alkenes in which nucleophiles other than O-nucleophiles could be employed. Accordingly, owing to the important properties of organoazides in pharmaceutical and agricultural sectors, Liu and coworkers ${ }^{[109]}$ recently disclosed an efficient copper-catalyzed trifluoromethylazidation of alkenes. In that work, a series of olefin derivatives 139 were trifluoromethylazidated with the aid of Togni II reagent (XVII) and catalytic amounts of $\mathrm{Cu}(\mathrm{I})$ salt at room temperature. In this transformation, 2 equiv of $\mathrm{TMSN}_{3}$ served as the source of the $\mathrm{N}_{3}$ motif. The substrate scope of the present transformation is remarkably broad. For example, not only styrene derivatives were transformed but also cyclic and acyclic internal olefins delivered the corresponding trifluoromethyl azides in good yields. Moreover, monosubstituted
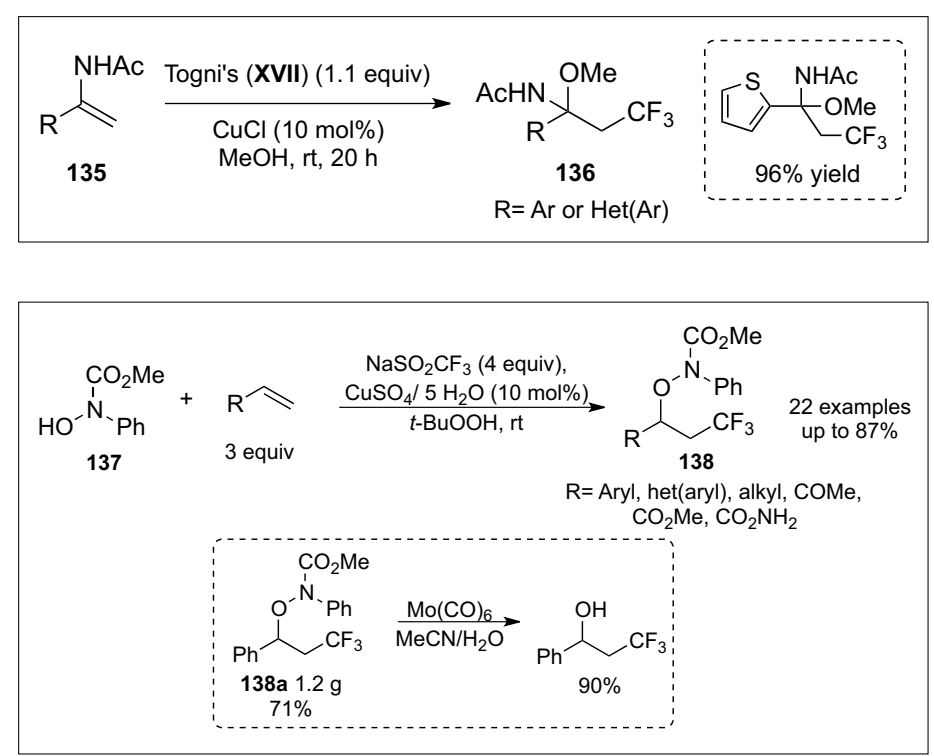

Scheme 77

Oxytrifluoromethylation of enamides.

Scheme 78 Oxytrifluoromethylation of alkenes with $\mathrm{N}$-hydroxycarbamate. 
and 1,1-disubstituted alkenes were also smoothly transformed. Of particular significance is the fact that $\alpha, \beta$-unsaturated carbonyl compounds were also suitable substrates. In this fashion, with the aid of a chiral auxiliary, they were able to synthesize precursors of type $140 \mathrm{~b}$, which could be further transformed into the corresponding $\beta-\mathrm{CF}_{3}-\alpha$-amino acids $141 \mathrm{~b}$. To further illustrate the synthetic applicability, the same group synthesized a series of complex olefins including a steroidal derivative, which successfully delivered compound 140a in good yield and good diastereoselectivity (Scheme 79).

\subsubsection{Trifluoromethylthiolation Reactions: Direct Introduction of $\mathrm{SCF}_{3}$ Fragments}

As previously mentioned, the $\mathrm{SCF}_{3}$ motifs have attracted a great deal of attention for a very long time. Since the preparation of $\mathrm{CuSCF}_{3}$ by Yagupolskii,,72] a great amount of work has been focused on the field. Recently, milder and more efficient methods and reagents that allow the direct trifluoromethyl-chalcogenation have been reported. ${ }^{[67,68]}$ The current methods for such transformation could be categorized in: a) nucleophilic, where a $\mathrm{CF}_{3} \mathrm{~S}^{-}$anion is the putative active species and b) electrophilic, in which a ' $\mathrm{SCF}_{3}{ }^{+}$' source is involved in the bond formation. Some common sources of $\mathrm{SCF}_{3}$ moiety are shown in Fig. 3.

\subsubsection{Nucleophilic \\ Trifluoromethylthiolation}

The most recent advances in nucleophilic trifluoromethylthiolation are based in transition metal-catalyzed cross-coupling protocols, in which copper-catalyzed/mediated protocols represent the vast majority of available methods. Nevertheless, reports utilizing other transition metals such as $\mathrm{Pd}{ }^{[110]}$ and $\mathrm{Ag}$ have also been published (vide supra). Only the most recent works will be discussed here. For a more comprehensive treatment, the reader is encouraged to consult other specialized reviews. ${ }^{[67,68]}$

Recently Qing and coworkers ${ }^{[11]}$ utilized a strategy previously reported by Y. L. Yagupolskii, ${ }^{[112]}$ in which the ${ }^{-} \mathrm{SCF}_{3}$ anion is prepared in situ from elemental sulfur and $\mathrm{TMSCF}_{3}$. In his report, a series of arylboronic acids were converted into the corresponding trifluoromethylthioethers in good to excellent yields. A current limitation of such method is the employment of expensive $\mathrm{Ag}_{2} \mathrm{CO}_{3}$ as an oxidant (Scheme 80, path A).

An alternative approach was reported by Weng and Huang. ${ }^{[113]}$ Recently the team developed an air-stable $\mathrm{CuSCF}_{3}$ reagent XXIII possessing a bidentate chelating nitrogen ligand which was efficiently used to perform trifluoromethylthiolation of aryl electrophiles. In such transformation

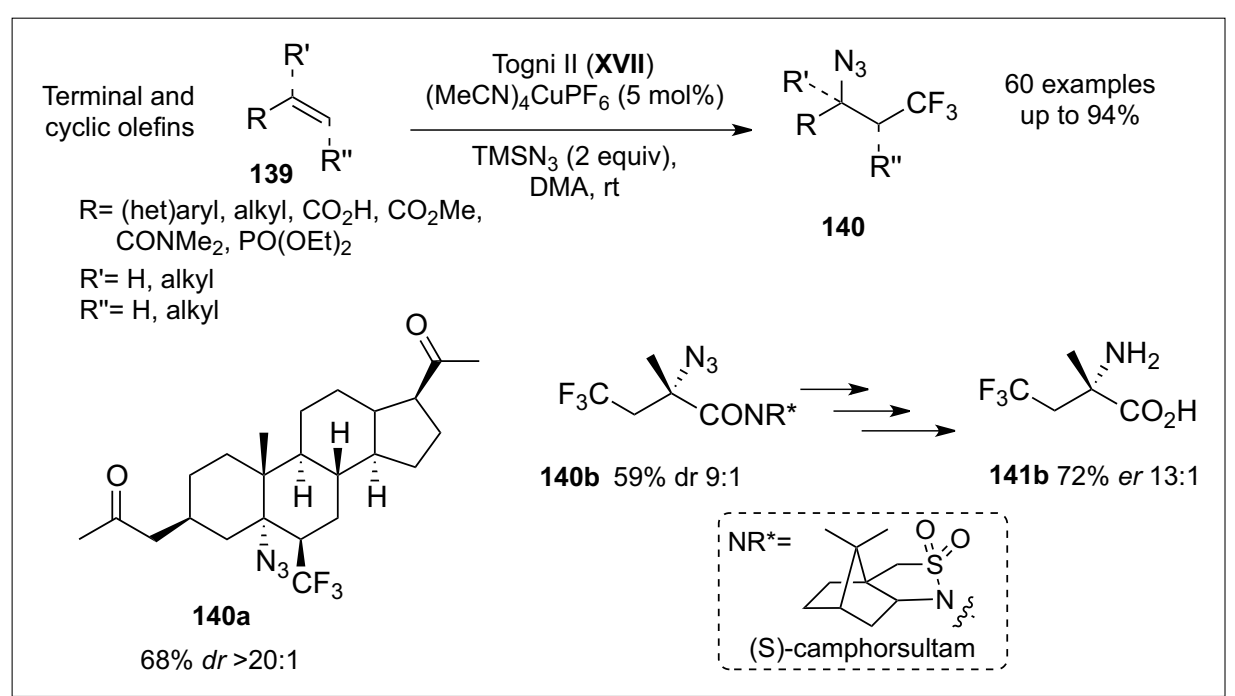

Scheme 79. Trifluoromethylazidation of alkenes.

Nucleophilic $\mathrm{SCF}_{3}$ sources

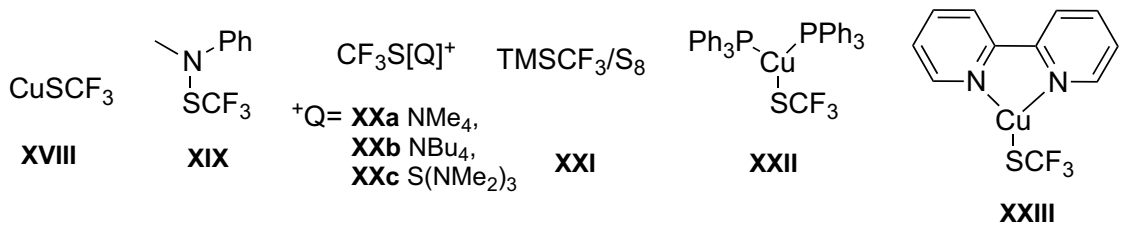

Radical or electrophilic $\mathrm{SCF}_{3}$ sources

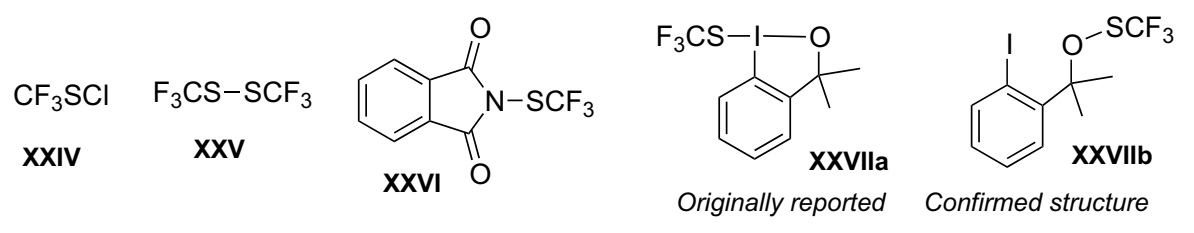

Fig. 3. Nucleophilic, radical or electrophilic $\mathrm{SCF}_{3}$ sources.

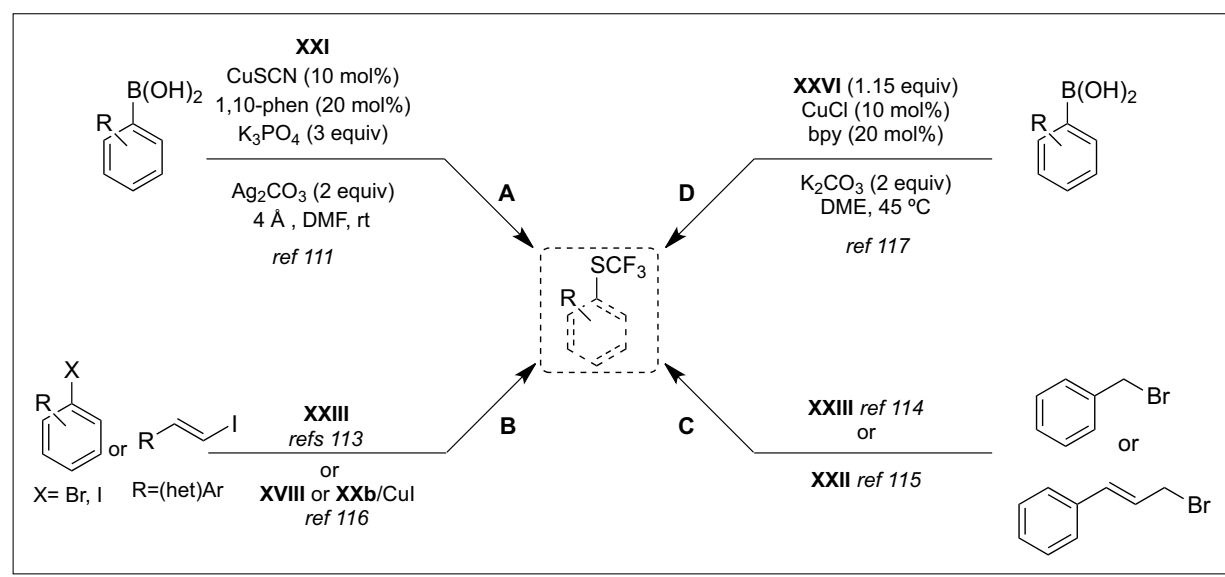

Scheme 80. Some nucleophilic and electrophilic trifluoromethylthiolation reactions.

aryl iodides as well as aryl bromides were successfully transformed (Scheme 80, path B). Remarkably, benzyl bromides ${ }^{[14]}$ were also suitable substrates and the corresponding products were delivered in good to excellent yields. Furthermore, expanding upon the above methodology, the same team prepared a complex bearing triphenyl phosphine as ligand and efficiently utilized it to effect trifluoromethylthiolation of allylic bromides ${ }^{115]}$ (Scheme 80, path C). Although the main drawback of such a method involves the use of stoichiometric amounts of copper, the enhanced reactivity and such well-defined systems is greatly showcased by their ability to transform such challenging substrates.

Alternatively, Rueping and coworkers $^{[116]}$ disclosed a procedure for the synthesis of vinyl-trifluoromethylthioethers 
through the reaction of XVIII and the corresponding vinyl iodides as the electrophilic coupling partner in pyridine as solvent. In this report, the team also applied catalytic quantities of $\mathrm{CuI}$ (ca. $10 \mathrm{~mol} \%$ ) and reagent $\mathbf{X X b}$, albeit with longer reaction times (Scheme 80, path B).

Very recently, the same team ${ }^{[117]}$ disclosed a new synthetic procedure to access the previously reported reagent XXVI, which avoids the use of the gaseous and toxic XXIV. In this report, XXVI was conveniently prepared by a metathetical reaction of $\mathrm{N}$-chlorophtalimide and $\mathrm{CuSCF}_{3}$. Next, reagent XXVI was successfully employed to perform a copper-catalyzed trifluoromethylthiolation of arylboronic acids in good yields (Scheme 80, path D). It should be mentioned that the team also utilized XXVI to perform the same transformation to alkynes.

In recent years, owing to their synthetic potential, several benziodioxole and benziodioxolone-based hypervalent iodine compounds have received great attention from the research community. ${ }^{[118,119]}$ Along these lines, the group of Shen ${ }^{[120]}$ recently disclosed a protocol in which an electrophilic $\mathrm{SCF}_{3}$ transfer reagent was successfully utilized to perform trifluoromethylthiolations of several nucleophiles. In this fashion, several enolates, alkynes and arylboronic acids were successfully transformed into the corresponding trifluoromethyl thioethers in good yields (Scheme 81).

Originally, the electrophilic reagent used in this work was proposed to consist of a sulfur-bound hypervalent iodine motif, such as the one depicted in XXVIIa (Fig. 3). However, as outlined by Buchwald and coworkers, ${ }^{[121]}$ thanks to a recently introduced crystalline sponge method for X-ray diffraction analysis, ${ }^{[122]}$ the structure of XXVIIa was unambiguously determined. The analysis demonstrated that the reagent possesses a thioperoxy framework such as the one depicted in XXVIIb, rather than the previously reported benziodoxole unit. In this report, the authors provide significant evidence by NMR analysis, which suggests that such a structure is still the prevalent one not only in the solid state, but also in solution. However, it must be further emphasized, that such findings do not in any form, affect the previously reported reactivity of the reagent (Scheme 81).

\subsection{Ruthenium-catalyzed Reactions}

Among the different processes catalyzed by ruthenium, metathesis reactions surely belong to the most important ones. Particularly, cross (CM) and ring-closing metathesis (RCM) have turned into some of the most efficient tools for the creation of $\mathrm{C}-\mathrm{C}$ bonds. ${ }^{[123]}$ As a result, $\mathrm{Ru}$-based metathesis catalysts have widely been used

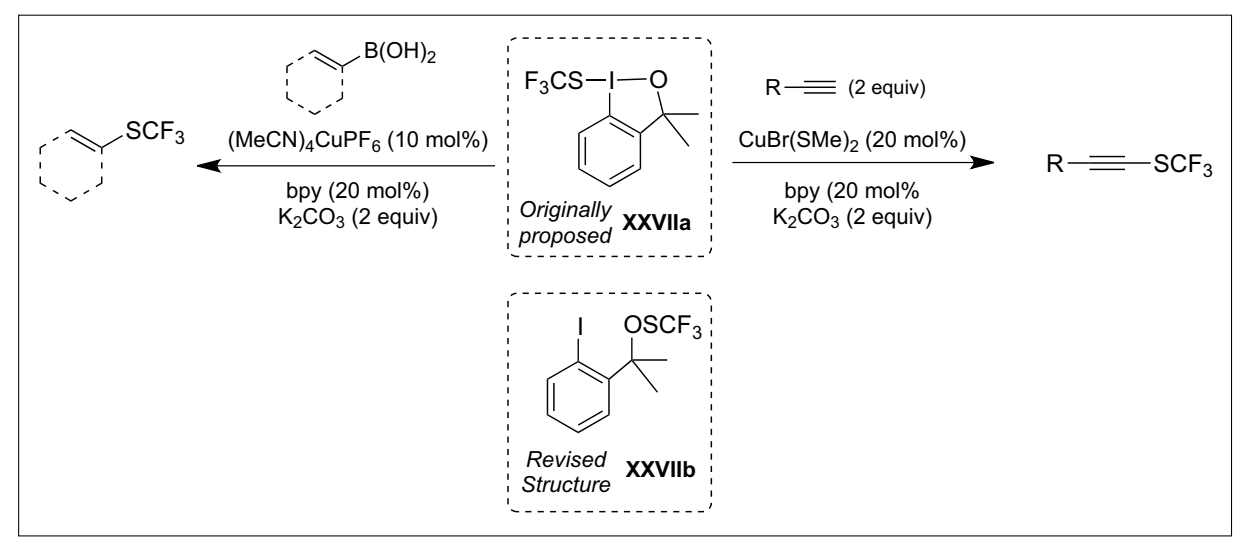

Scheme 81. Electrophilic trifluoromethylthiolation and revised structure of reagents.

to prepare a range of interesting synthetic and natural products including amino acids, carbo- and heterocycles, etc. Moreover, it is common to find in the literature many examples of metathesis, in particular enyne metathesis (EYM), in combination with Diels-Alder or Pauson-Khand reactions, for instance. However, examples that involve fluorinated substrates are lacking.

On these premises, Bonnet-Delpon and coworkers reported the synthesis of $\mathrm{CF}_{3}$-substituted piperidine derivatives 144 by Ru-catalyzed RCM from doubly unsaturated trifluoromethylated amines 143 (Scheme 82).[124] The starting fluorinated amines 143 were readily prepared through a one-pot, Zn-mediated $C$-allylation $/ N$ allylation sequence in moderate to good yields.
Then, RCM in the presence of Grubbs catalyst $[\mathbf{R u}-\mathbf{I}]$ was carried out under mild conditions leading to fluorinated piperidines 144 in excellent yields. Interestingly, when two enyne isomers, homopropargyl allyl amine 146 and homoallyl propargyl amine 145, were subjected to the optimized reaction conditions, only the latter underwent cyclization, providing the corresponding 1,3-diene 147 (Scheme 82). In 2012, Portella and coworkers developed a similar strategy for the synthesis of $\mathrm{CF}_{3}$-containing cyclopentenecarboxylic acid derivatives $\mathbf{1 4 8}$ as promising building blocks.[125] The derivatization of the final products would be a special feature of this work (Scheme 83).

On the other hand, Osipov and Dixneuf described the behavior of $\mathrm{N}$-tethered
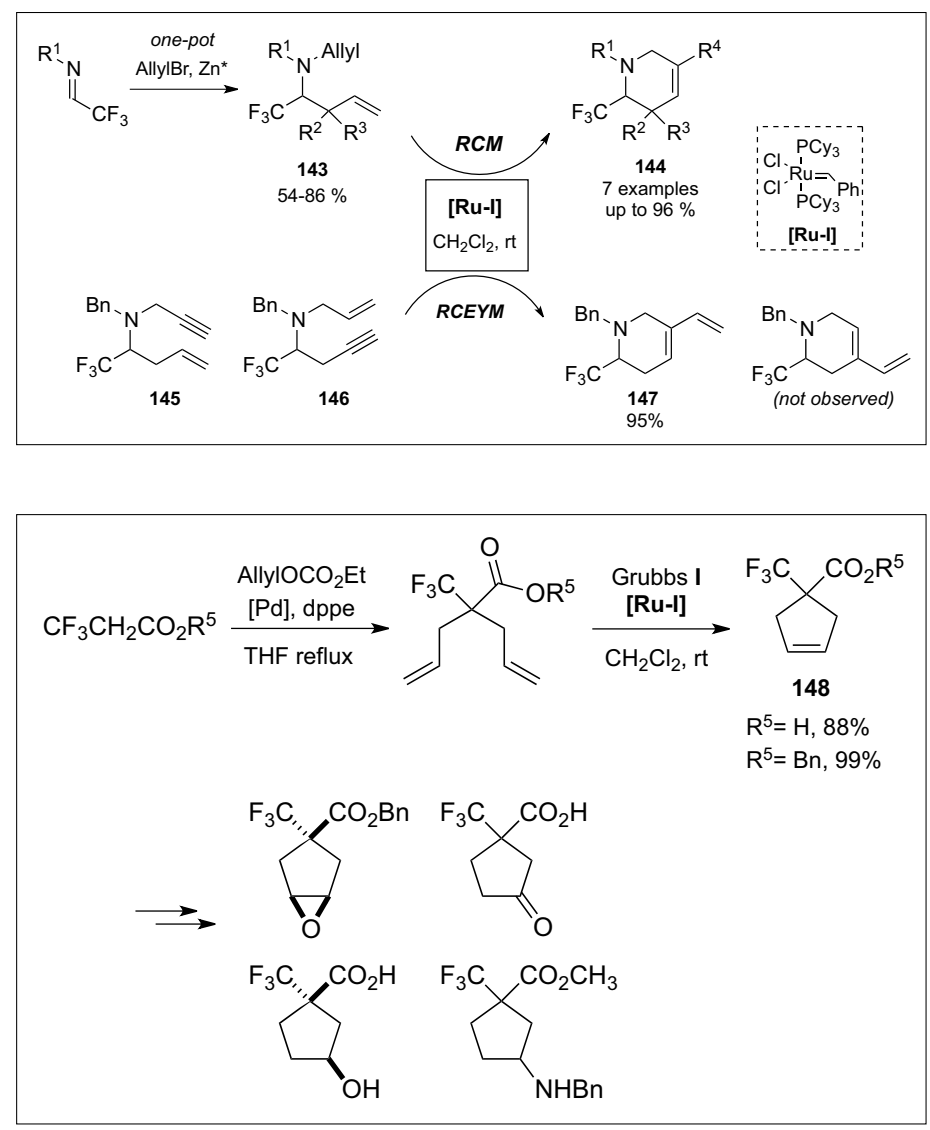

Scheme 82. Rucatalyzed RCM of doubly unsaturated trifluoromethylated amines 143.

Scheme 83.

Synthesis and derivatization of cyclopentenecarboxylic acid derivatives 148 . 
trifluoromethyl enynes towards ruthenium-based metathesis catalysts. ${ }^{[126]}$ The addition of vinyl- or allylmagnesium bromide to imines 149 followed by $\mathrm{N}$-propargylation afforded closely related 1,6- and 1,7-enynes 150 (Scheme 84). [126b,c] However, these intermediates were not used in 'normal' RCEYM. Instead, they were treated with the ruthenium pre-catalyst $[\mathrm{Cp} *(\mathrm{Cl}) \mathrm{Ru}(\mathrm{cod})]$, in the presence of a diazocompound, affording the first trifluoromethylated bicyclic proline and pipecolic acid derivatives $\mathbf{1 5 1}$, respectively. The only difference between this tandem carbene addition-cyclopropanation reaction and an RCEYM, besides the initial carbene insertion step, was the last step of the catalytic cycle based on intermediate A (Scheme 84). The authors suggested that the $\mathrm{Cp}^{*}(\mathrm{Cl}) \mathrm{Ru}$ moiety favors reductive elimination with respect to the (IMes) $\mathrm{Cl}_{2} \mathrm{Ru}$ moiety, finally leading to metathesis.

By using 1,7-enyne analogs with the aforementioned substrates 150, the same authors investigated their reactivity under RCEYM conditions. After observing that the RCEYM on terminal enyne $\mathbf{1 5 2}$ invariably led to the self-cross-metathesis of the RCEYM product in a significant ratio, the authors performed a Pd-catalyzed Sonogashira cross-coupling with different aryl iodides for the substitution on the triple bond (Scheme 85). Treatment of the new substituted enynes 153 with Grubbs catalyst [Ru-II] in toluene at $80^{\circ} \mathrm{C}$ afforded RCEYM products $\mathbf{1 5 4}$ in good yields (Scheme 85).

Interestingly, enynes derived from ortho-substituted aryl iodides were completely inert to RCEYM under different reaction conditions. The authors attributed this observation to a steric effect that would shield the triple bond, thus preventing coordination to the ruthenium center. Furthermore, terminal enyne $\mathbf{1 5 2}$ was cross-coupled with different acid chlorides affording enynones $\mathbf{1 5 5}$, which were in turn cyclized using Hoveyda-Grubbs catalyst [Ru-III], under an ethylene atmosphere to obtain 156 (Scheme 85).

In contrast to RCEYM, the intermolecular version, i.e. the cross-enyne metathesis (CEYM) reaction, has been much less applied in synthesis probably due to the difficulty of controlling $E / Z$ selectivity in its final products. Nevertheless, the beneficial effect of ethylene gas discovered by Mori et al..$^{[127]}$ revolutionized this issue, providing 1,3-dienes efficiently. To the best of our knowledge, only a few examples of CEYM involving fluorinated alkynes have been reported so far. ${ }^{[128]}$

Fustero et al. disclosed a tandem onepot CEYM/Diels-Alder reaction of difluoropropargylic alkynes with different dienophiles under Mori's conditions, giving

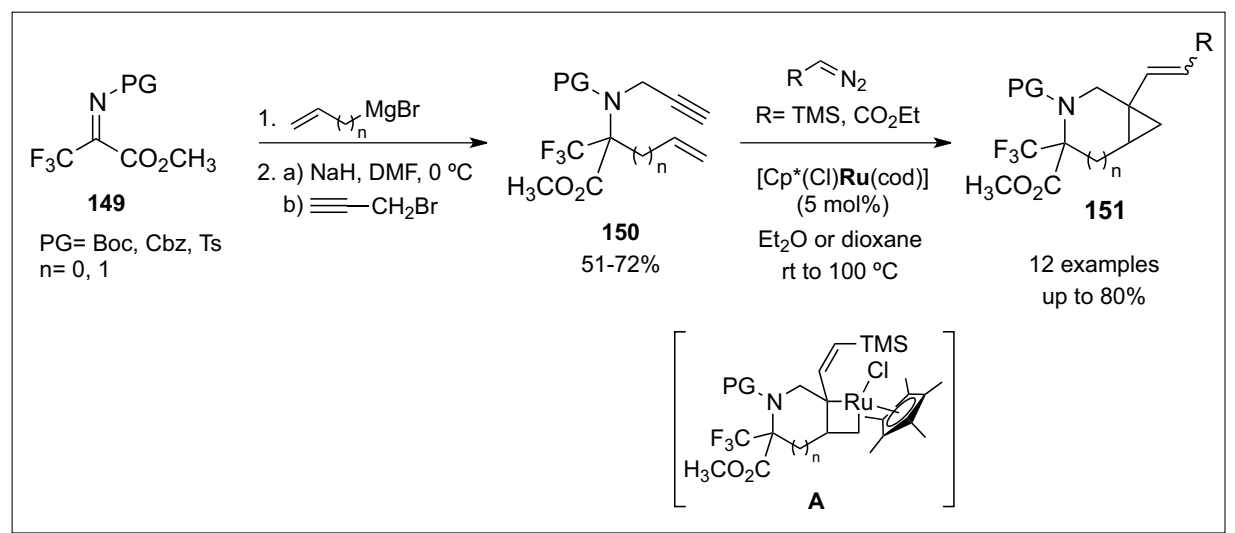

Scheme 84. Carbene insertion/cyclopropanation tandem reaction.

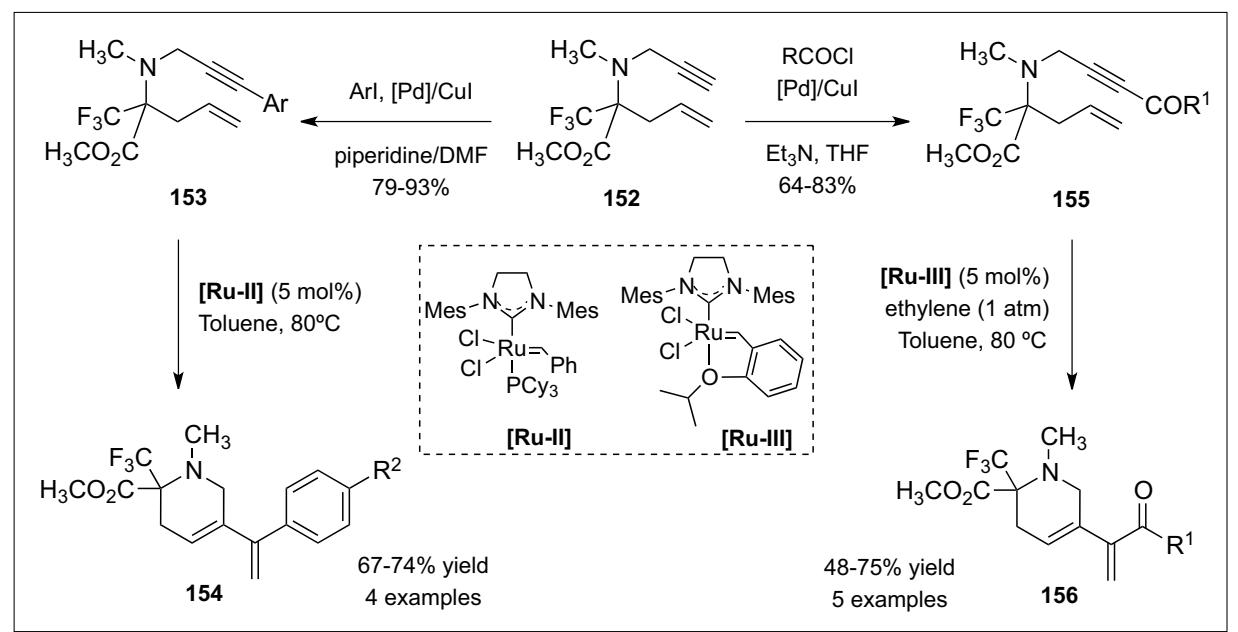

Scheme 85. RCEYM on substituted fluorinated 1,7-enynes. rise to fluorinated carbo- and heterocyclic derivatives in moderate to good yields (Scheme 86, pathway A).[128b] The one-pot protocol began with the ethylene-mediated CEYM on alkynes $\mathbf{1 5 7}$ in the presence of Hoveyda-Grubbs catalyst [Ru-III] leading to a full conversion into the diene intermediate 158. Then, dienophile was added at room temperature to initiate the subsequent Diels-Alder reaction.

As an alternative to Mori's conditions, the same authors reported a complementary methodology based on a tandem mul- ticomponent protocol in which 1,7-octadiene was used as an in situ source of ethylene gas (Scheme 86, pathway B). ${ }^{[128 c]}$ In this case, the ethylene released from the RCM of 1,7-octadiene activated the ruthenium precatalyst, which in turn reacted with the alkyne 157 triggering the tandem CEYM/Diels-Alder reaction sequence.

A different tandem process involving cross metathesis but, this time, in combination with aza-Michael addition, was reported by Fustero et al. Based upon previous work using carbamates ${ }^{[129 a]}$ or

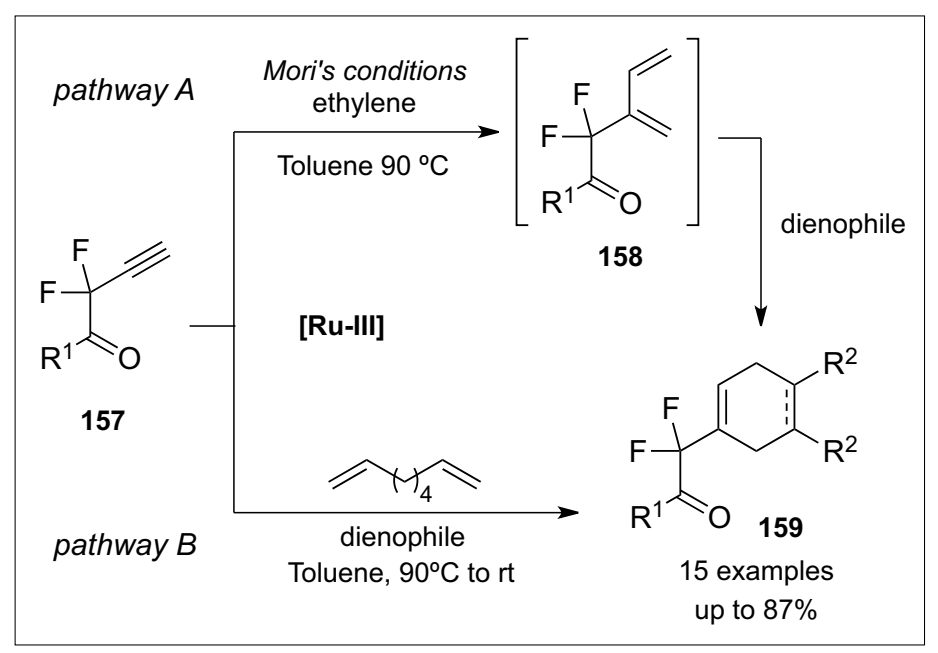

Scheme 86.

Sequential CEYM/

Diels-Alder reaction. 
N-sulfinyl amines ${ }^{[129 b]}$ as nucleophilic nitrogen sources, a tandem CM/intramolecular aza-Michael reaction (IMAMR) was performed on $\alpha, \alpha$-difluorinated amides 160, leading to interesting scaffolds such as fluorinated $\gamma$ - and $\delta$-lactams 162 (Scheme 87). ${ }^{[130]}$

In a tandem fashion, fluorinated amides 160 reacted with methyl vinyl ketone 161a in the presence of Hoveyda-Grubbs catalyst [Ru-III] and titanium(IV) tetraisopropoxide as a co-catalyst, leading to the corresponding lactams 162 by IMAMR in good yields. In contrast to ketones, esters derived from ethyl acrylate 161b stopped at the CM step (163) and the addition of potassium tert-butoxide was required to promote cyclization (Scheme 87).

One of the best ways of revealing the distinct reactivity of fluorine-containing molecules is to compare them with their non-fluorinated analogs. Thus, Hoff and coworkers exposed these differences between aryl fluorinated and non-fluorinated ketones in Ru-catalyzed asymmetric transfer hydrogenation (ATH). ${ }^{[131]}$ A range of aryl fluoromethyl ketones $\mathbf{1 6 4}$ were subjected to reduction with complex $\mathbf{I}$, observing that the gradual introduction of fluorine atoms into the $\alpha$-methyl substituent of the ketone accelerated the reaction significantly and improved conversion into 165 (>99\%) in most cases. However, a sharp decrease of enantioselectivity was observed for trifluoroketones (Scheme 88).

The authors attributed the lower selectivity mainly to a size effect, i.e. an increase in the size of the fluoroalkyl group. Also, an additional electronic effect caused by the aromatic group was suggested by calculations; this effect would play an important role in the $\pi-\pi$ interaction between the catalyst and the substrate.

Another example was recently reported by Ikariya and coworkers in the synthesis of $\alpha$-fluorinated alcohols 167 and hemiacetals 168 from fluorinated esters 166 via ruthenium-catalyzed selective hydrogenation (Scheme 89). ${ }^{[132]}$ It is noteworthy that the hydrogenation of $\beta$-fluorinated esters or non-fluorinated methyl acetate were unsuccessful. Moreover, aliphatic $\alpha$-monofluoro esters were hydrogenated in good yields but decreasing selectivity probably due to the epoxidation of the resulting monofluorinated alcohol. Although the reaction proceeded under mild conditions, the process exhibited a strong dependence on the reaction conditions as any change in temperature, amount of base, catalyst or hydrogen pressure modified the product ratio of $\mathbf{1 6 7 / 1 6 8}$.

The behavior of $\mathrm{CF}_{3}$-substituted allenes in the presence of a ruthenium complex were studied by Krische and coworkers. ${ }^{[133]}$ Furthermore, an effective synthesis

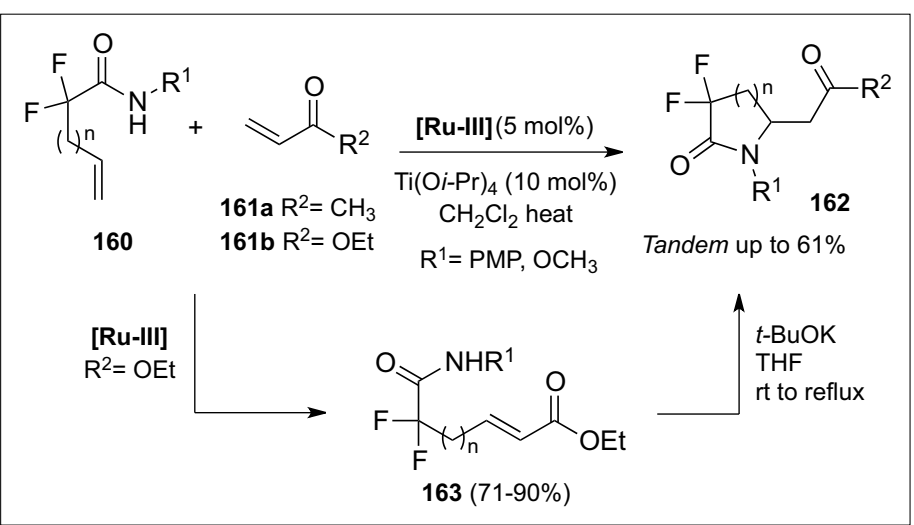

Scheme 87.

Tandem CM-IMAMR sequence.

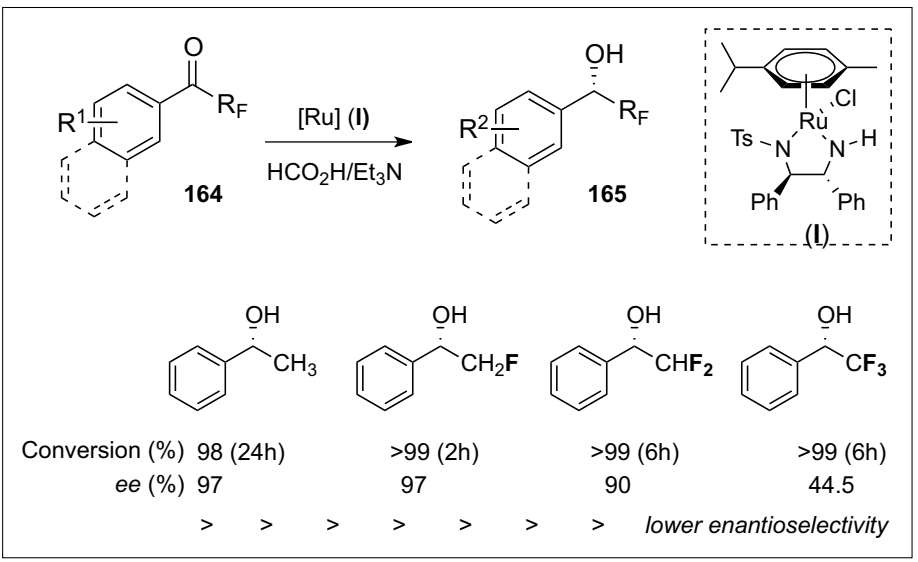

Scheme 88.

Asymmetric reduction of fluorinated ketones using Ru-based catalysts.

Scheme 89. Selective hydrogenation of $\alpha$-fluorinated esters catalyzed by Ru(II) catalysts 169 .

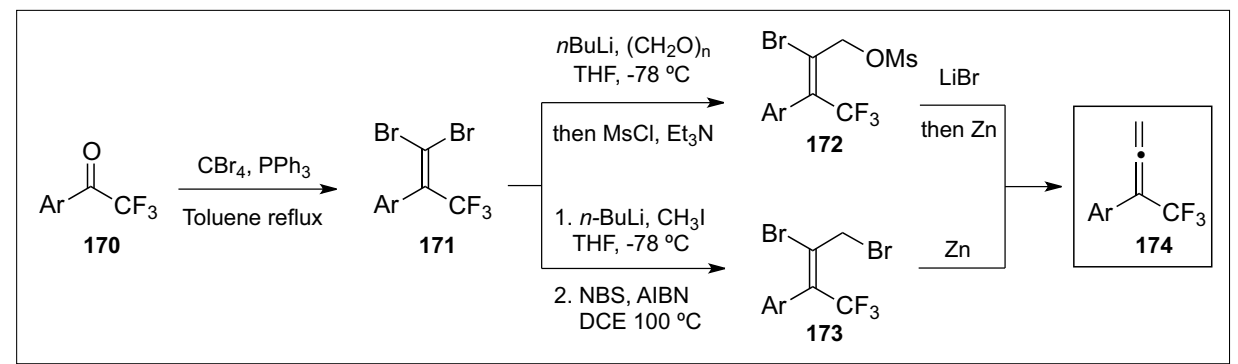

Scheme 90. Synthesis of $\mathrm{CF}_{3}$-substituted allenes 174.

of 1-aryl-1-trifluoromethylallenes $\mathbf{1 7 4}$ was performed starting from trifluoromethyl ketones 170 (Scheme 90), which underwent olefination to give the corresponding methylene dibromides 171. Through two equivalent pathways, the latter was transformed into the allylic bromide $\mathbf{1 7 2}$ and/ or $\mathbf{1 7 3}$ and subsequent treatment with zinc dust led to the expected allenes $\mathbf{1 7 4}$.

The optimized combination of $\mathrm{RuHCl}(\mathrm{CO})\left(\mathrm{PPh}_{3}\right)_{3}$ as a precatalyst and DPPM as a ligand catalyzed efficiently the reductive coupling of 1-aryl-1-trifluoromethylallenes 174 with paraformaldehyde to give $\mathrm{CF}_{3}$-containing homoallylic alcohols 175 (Scheme 91), involving the formation of a new quaternary stereocenter. The authors proposed a mechanism based on allylruthenium species Ia-Ib as the key intermediates in the catalytic cycle. To complement this study, the final synthon 175 was employed as building block for the synthesis of different substrates such as the fluorinated nitrile $\mathbf{1 7 6}$ and the methyl ester 177 (Scheme 91).

On the other hand, an efficient, regioselective cyclotrimerization of trifluoromethylated internal alkynes $\mathbf{1 7 8}$ catalyzed 
by a $\mathrm{Ru}_{3}(\mathrm{CO})_{12} / 2$-DPPBN [2-(diphenyl phosphine)benzonitrile] catalytic system was described by Kawatsura et al. (Scheme 92). Although this reaction had already been reported previously using nickel ${ }^{[134 a]}$ and rhodium ${ }^{[134 b]}$ catalysis, this example was the first one catalyzed by a ruthenium complex.

Optimization revealed that $\mathrm{Ru}_{3}(\mathrm{CO})_{12}$ as a precatalyst with 2-DPPBN phosphine in a 2:1 specific ratio was the perfect combination for providing unsymmetrical fluorinated benzene derivatives $\mathbf{1 7 9}$ in high yields and excellent regioselectivities. The ruthenacyclopentadiene species $\mathbf{A}$ was identified as the active catalyst-intermediate (Scheme 92). In general, this method tolerated different substituents at the aromatic moiety of the alkyne; however, electron-withdrawing groups slightly favored the formation of symmetrical products 180. A steric effect of an ortho-substituted aromatic group was also observed, inhibiting the process.

Up to now, we have reviewed the most recent advances in transition metal-catalyzed trifluoromethylations. Besides palladium, silver or copper, ruthenium is also able to participate in such a reaction. In general, the ruthenium complex catalyzes the redox formation of the electrophilic trifluoromethyl radical $\left({ }^{\circ} \mathrm{CF}_{3}\right)$, which is indeed the active species in the addition to the substrate.

In this context, Zakarian and coworkers described the diastereoselective addition of ${ }^{\circ} \mathrm{CF}_{3}$ to $\mathrm{N}$-acyl oxazolidinones 181 via in situ generated zirconium enolates. ${ }^{[135]}$ In contrast to previously reported $\alpha$-trichloromethylation of titanium enolates, ${ }^{[136]} \mathrm{TiCl}_{4}$ was not effective for fluoroalkylation and other metal halides were screened. The optimized reaction conditions established $\mathrm{ZrCl}_{4}$ and $\mathrm{HfCl}_{4}$ as the metal halides of choice for enolization of 181, $\mathrm{Ru}\left(\mathrm{PPh}_{3}\right)_{3} \mathrm{Cl}_{2}$ as the best catalyst and trifluoromethyl or perfluoroalkyl/benzyl iodides $\left(\mathrm{R}_{\mathrm{F}} \mathrm{I}\right) \mathbf{1 8 2}$ as the fluoroalkyl radical source (Scheme 93).

Additionally, Zakarian and coworkers demonstrated by NMR experiments the formation in situ of zirconium enolates 184 by reaction of 181 with $\mathrm{ZrCl}_{4}$ and $\mathrm{Et}_{3} \mathrm{~N}$ at room temperature. The freshly formed enolate was then trapped by ${ }^{\circ} \mathrm{CF}_{3}$ species, which were in turn generated from iodotrifluoromethane $\mathbf{1 8 2}$ by a Ru(II)-catalyzed redox process (Scheme 93). A final single electron transfer from intermediate II, in equilibrium with I, to $\mathrm{Ru}(\mathrm{III})$ complex led to the final $\mathrm{CF}_{3}$-substituted oxazolidinone $\mathbf{1 8 3}$, initiating a new catalytic cycle.

The group of Gouverneur have perfectly combined this kind of process with the effect of visible-light on ruthenium catalysts, i.e. photoredox catalysis. ${ }^{[137]}$ Thus, the photoredox-based trifluoromethylation

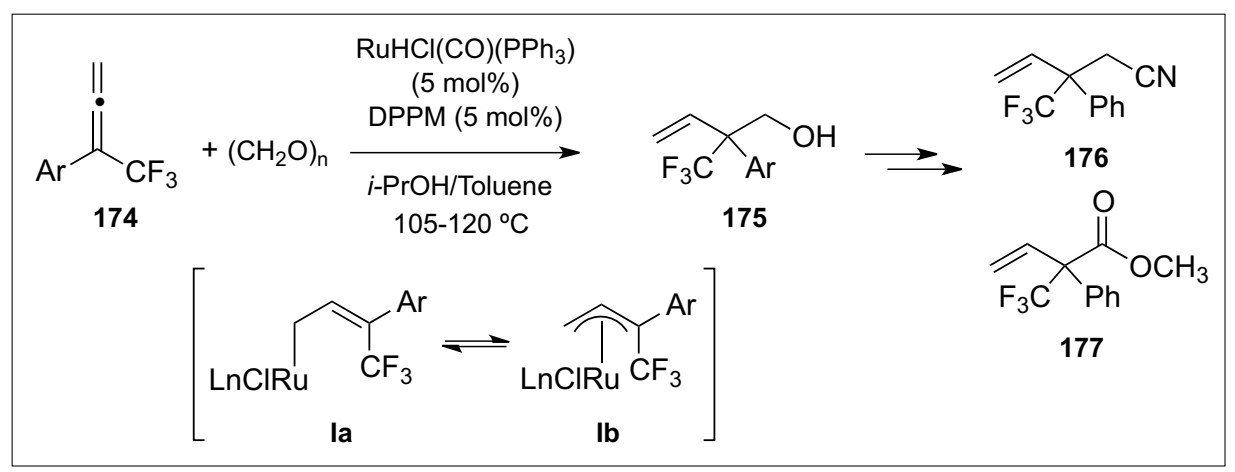

Scheme 91. Ru-catalyzed reductive coupling of $\mathrm{CF}_{3}$-containing allenes to paraformaldehyde.

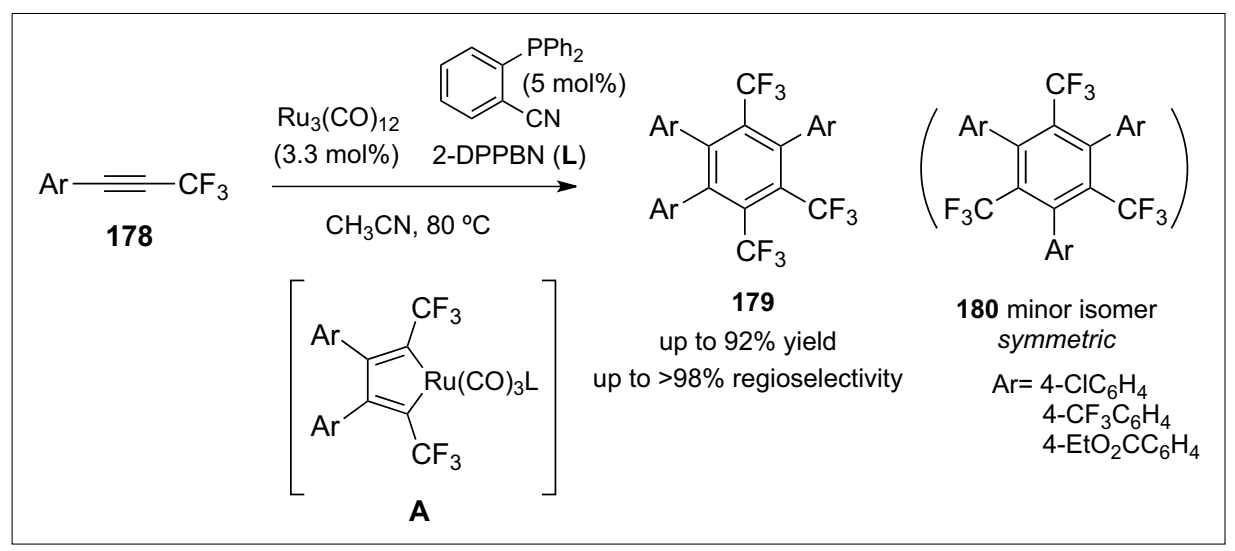

Scheme 92. Ru-catalyzed regioselective cyclotrimerization of trifluoromethylated alkynes.

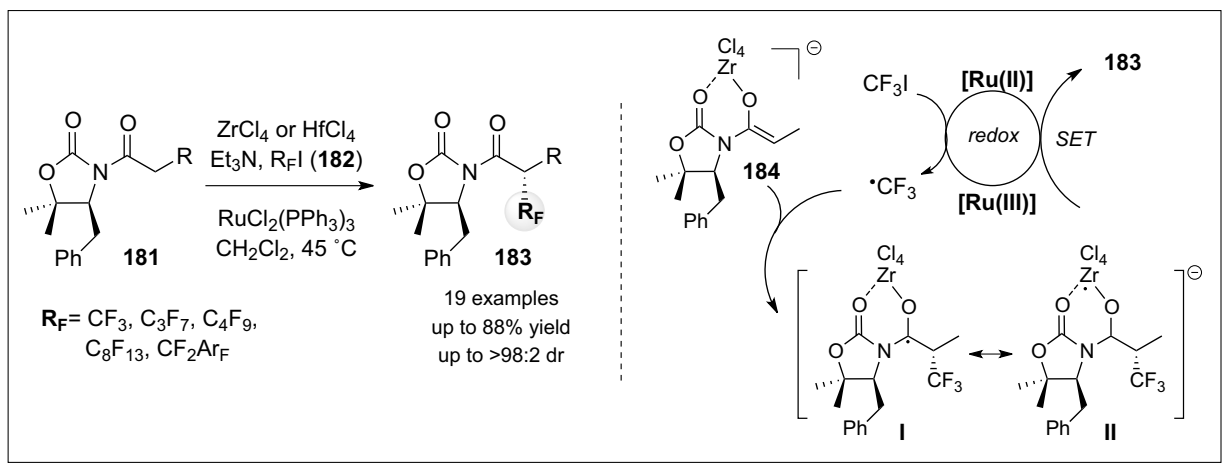

Scheme 93. Asymmetric trifluoromethylation of $N$-acyl oxazolidinones.

of allylsilanes 185 was developed under mild conditions, employing Togni reagent XVII or Umemoto reagent XV (Fig. 2) as

$\mathrm{CF}_{3}$ radical source. ${ }^{[137 \mathrm{a}]}$ The photoredox catalyst of choice was $\mathrm{Ru}(\text { bpy })_{3} \mathrm{Cl}_{2} \cdot 6 \mathrm{H}_{2} \mathrm{O}$ which, under visible-light irradiation, re-

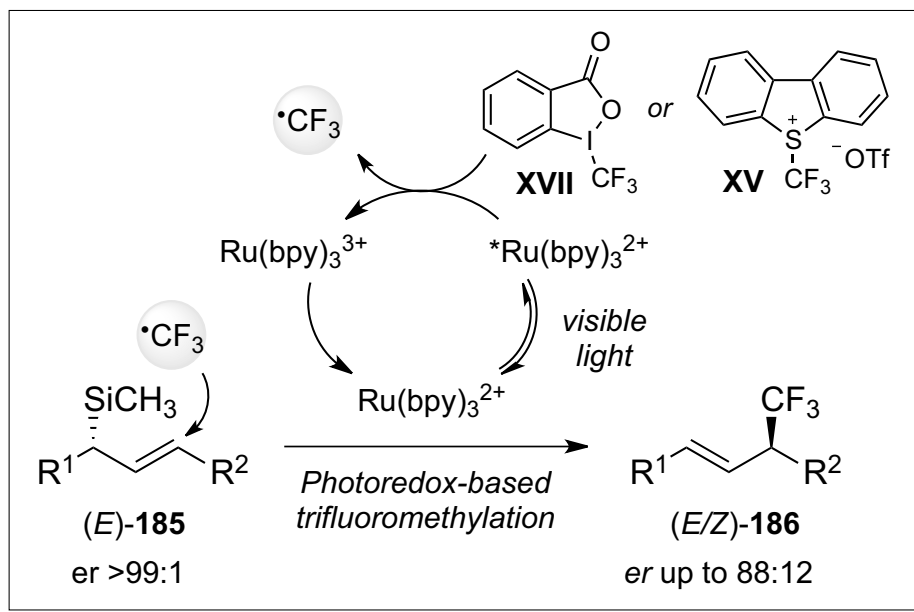

Scheme 94. Photoredox-based trifluoromethylation of allylsilanes 185. 
sulted in excited-state $\left[\mathrm{Ru}^{*}\right]$-species involved in ${ }^{\circ} \mathrm{CF}_{3}$ generation (Scheme 94).

Arguably, the silyl group in the starting substrate would be the steering group since it was crucial to get good regio- and stereo$\mathrm{CF}_{3}$ source was found to be determining on reactivity as well as stereoselectivity. In general, this method allowed the synthesis of allylic $\mathrm{CF}_{3}$ products 186 as the $E$ isomer as the major product by using Togni reagent XVII (Fig. 2). However, Umemoto reagent $\mathbf{X V}$ provided higher $E$-selectivity to the detriment of the chemical yield. Furthermore, trifluoromethylation as well as addition of other fluorine grouping (e.g. $\mathrm{CF}_{2} \mathrm{CF}_{3}$ group) were successfully performed on enantioenriched substrates, although a loss of the optical purity was observed for the $E$-isomer.

The same authors also worked on the net fluoroform $\left(\mathrm{CF}_{3} \mathrm{H}\right)$ addition to alkenes and alkynes employing separately $\mathrm{CF}_{3}$ radical and hydrogen atom sources, i.e. Umemoto reagent $\mathbf{X V}$ and methanol respectively. ${ }^{[137 b]}$ Besides being the solvent of the reaction, the role of methanol as a hydrogen donor should be particularly highlighted since an external hydrogen source such as Hantzsch ester was not required. Thus, the hydrotrifluoromethylation would be based on a similar mechanism to the aforementioned (Scheme 95) in which a Ru-based photoredox process generated ${ }^{\circ} \mathrm{CF}_{3}$ species to be added regioselectively to the alkene/alkyne $\mathbf{1 8 7 / 1 8 8}$. Final oxidation of methanol by the strong oxidant $\mathrm{Ru}(\mathrm{bpy})_{3}{ }^{3+}$ regenerated the catalyst on one hand and, on the other hand, it transferred a hydrogen atom to the radical intermediate $\mathbf{1 8 9}$ leading to the expected terminal $\mathrm{CF}_{3}$-substituted compounds 190 and/or 191.

\section{Conclusions}

This review aims to give a systematic overview of the most relevant aspects of organofluorine chemistry and some of the most representative transition metals such as $\mathrm{Co}, \mathrm{Au}, \mathrm{Pd}, \mathrm{Ru}$, and in greater detail, selectivity. Additionally to this effect, the

$\mathrm{Ag}$ and $\mathrm{Cu}$. More specifically, the unique, differential reactivity of these transition metals towards organofluorine compounds have received the most attention. Because of the tremendous advances achieved in this field over the last few years, this review may provide valuable complementary information on this issue, including preferably those examples reported in the last three years. Indeed, this promising field is significantly growing, as much in academy as in industry and, as a result, a future review about the further developments in this area should be considered in a short time.

\section{Acknowledgments}

We thank the Spanish Ministerio de Ciencia e Innovación (CTQ2010-19774C02-01) and the Generalitat Valenciana (PROMETEO/2010/061) for their financial support. S.C. thanks the Spanish government for a Juan de la Cierva contract.

\section{Received: March 27, 2014}

[1] a) J.-P. Bègué, D., Bonnet-Delpon, 'Bioorganic and Medicinal Chemistry of Fluorine', Wiley, Hoboken, NJ, 2008; b) K. Müller, C. Faeh, F. Diederich, Science 2007, 317, 1881; c) S. Purser, P. R. Moore, S. Swallow, V. Gouverneur, Chem. Soc. Rev. 2008, 37, 320; d) T. Liang, C. N. Neumann, T. Ritter, Angew. Chem. Int. Ed. 2013, 52, 8214 and references therein

[2] D. O'Hagan, Chem. Soc. Rev. 2008, 37, 308.

[3] a) D. J. O'Hagan, J. Fluorine Chem. 2010, 131, 1071; b) S. R. Pattan, N. S. Dighe, H. V. Shinde, M. B. Hole, V. M. Gavare, Asian J. Res. Chem. 2009, 2, 376; c) I. Ojima, 'Fluorine in medicinal chemistry and chemical biology', Wiley-Blackwell, Chichester, 2009; d) W. K. Hagmann, J. Med. Chem. 2008, 51, 4359; e) K. L. Kirk, Org. Proc. Res. Devel. 2008, 12, 305.

[4] R. Filler, R. Saha, Future Med. Chem. 2009, 1, 777.

[5] a) Z. Jin, G. B. Hammond, B. Xu, Aldrichimia Acta 2013, 45, 67; b) T. Besset, C. Schneider, D. Cahard, Angew. Chem. Int. Ed. 2012, 51, 5048; c) C. Hollingworth, V. Gouverneur, Chem. Commun. 2012, 48, 2929; d) T. Furuya, A. S. Kamlet, T. Ritter, Nature 2011, 473, 470; e) K. M. Engle, T.-S. Mei, X. Wang, J.-Q. Yu, Angew. Chem. Int. Ed. 2011, 50, 1478; f) O. A. Tomashenko, V. V. Grushin, Chem. Rev. 2011 , 111,$4475 ;$ g) M. N. Hopkinson, A. D. Gee, V. Gouverneur, Isr. J. Chem. 2010, 50, 675; h) R. J.

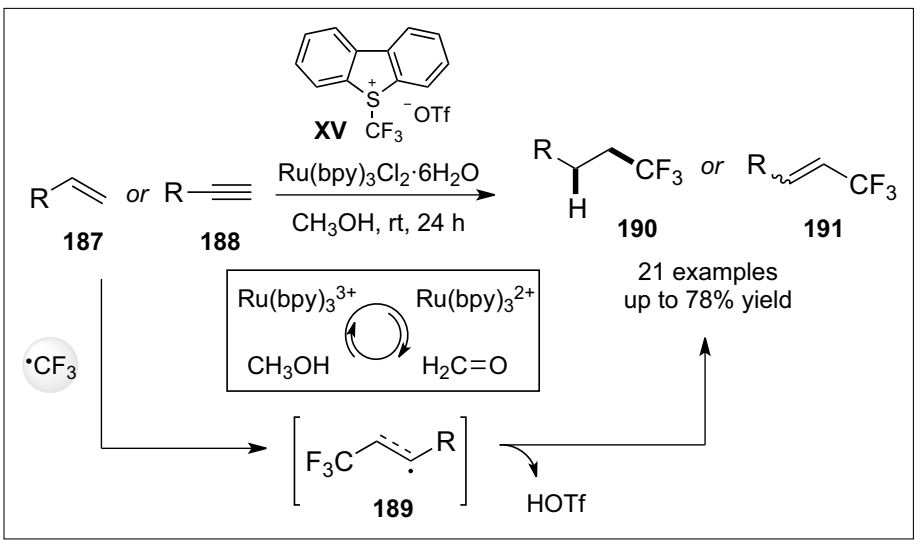

Lundgren, M. Stradiotto, Angew. Chem. Int. Ed. 2010, 49, 9322.

[6] R. Ríos Torres, in 'The Pauson-Khand Reaction', Ed. R. Ríos Torres, Wiley-VCH, Weinheim, 2012

[7] J. Kizirian, N. Aiguabella, A. Pesquer, S. Fustero, P. Bello, X. Verdaguer, A. Riera, Org. Lett. 2010, 12, 5620.

[8] N. Aiguabella, C. del Pozo, X. Verdaguer, S. Fustero, A. Riera, Angew. Chem. Int. Ed. 2013, $52,5355$.

[9] T. Konno, T. Kida, A. Tani, T. Ishihara, J. Fluorine Chem. 2012, 144, 147

[10] N. Aiguabella, E. M. Arce, C. del Pozo, X. Verdaguer, A. Riera, Molecules 2014, 19, 1763.

[11] R. Nadano, J. Ichikawa, Chem. Lett. 2007, 36, 22.

[12] S. Fustero, R. Lázaro, N. Aiguabella, A. Riera, A. Simón-Fuentes, P. Barrio, Org. Lett. 2014, 16, 1224.

[13] a) G. Zhang, L. Cui, Y. Wang, L. Zhang, J. Am. Chem. Soc. 2010, 132, 1474; b) G. Zhang, Y. Peng, L. Cui, L. Zhang, Angew. Chem. Int. Ed. 2009, 48, 3112; c) Y. Peng, L. Cui, G. Zhang, L. Zhang, J. Am. Chem. Soc. 2009, 131, 5062; d) W. E. Brenzovich, Jr. D. Benitez, A. D. Lackner, H. P. Shunatona, E. Tkatchouk, W. A. Goddard III, F. D. Toste, Angew. Chem. Int. Ed. 2010, 49, 5519; e) A. D. Melhado, W. E. Brenzovich, A. D. Lackner, F. D. Toste, J. Am. Chem. Soc. 2010, 132, 8885 .

[14] W. Wang, J. Jasinski, G. B. Hammond, B. Xu, Angew. Chem. Int. Ed. 2010, 49, 7247.

[15] N. P. Mankad, F. D. Toste, J. Am. Chem. Soc. 2010, 132, 12859.

[16] T. de Haro, C. Nevado, Chem. Commun. 2011, 47, 248

[17] M. N. Hopkinson, G. T. Giuffredi, A. D. Gee, V. Gouverneur, Synlett 2010, 18, 2737.

[18] Z. Jin, R. S. Hidinger, B. Xu, G. B. Hammond, J. Am. Chem. Soc. 2012, 77, 7725.

[19] a) A. Arcadi, E. Pietropaolo, A. Alvino, V. Michelet, Beilstein J. Org. Chem. 2014, 10, 449; b) A. Arcadi, E. Pietropaolo, A. Alvino, V. Michelet, Org. Lett. 2013, 15, 2766.

[20] S. Fustero, P. Bello, J. Miró, M. SánchezRoselló, M. A. Maestro, J. González, C. del Pozo, Chem. Comm. 2013, 49, 1336.

[21] S. Fustero, I. Ibáñez, P. Barrio, M. A. Maestro, S. Catalán, Org. Lett. 2013, 15, 832.

[22] G. T. Giuffredi, B. Bernet, V. Gouverneur, Eur. J. Org. Chem. 2011, 20-21, 3825.

[23] C. Hollingworth, A. Hazari, M. N. Hopkinson, M. Tredwell, E. Benedetto, M. Huiban, A. D. Gee, J. M. Brown, V. Gouverneur, Angew. Chem. Int. Ed. 2011, 50, 2613.

[24] M. H. Katcher, A. G. Doyle, J. Am. Chem. Soc. 2010, 132, 17402.

[25] M. H. Katcher, A. Sha, A. G. Doyle, J. Am. Chem. Soc. 2011, 133, 15902.

[26] For some recent reviews on C-H activation, see: a) J. Wencel-Delord, T. Dröge, F. Liu, F. Glorius, Chem. Soc. Rev. 2011, 40, 4740; b) O. Baudoin, Chem. Soc. Rev. 2011, 40, 4902; c) J. WencerDelord, F. Glorius, Nature Chemistry 2013, 5, 369; d) S. I. Kozhushkov, L. Ackermann, Chem. Sci. 2013, 4, 886. For C-H fluorination, see: e) K. S. L. Chan, M. Wasa, X. Wang, J.-Q Yu, Angew. Chem. Int. Ed. 2011, 50, 9081; f) S.-J. Lou, D.-Q. Xu, A.-B. Xia, Y.-F. Wang, Y.K. Liu, X.-H. Du, Z.-Y. Xu, Chem. Commun. 2013, 49, 6218; g) T. Truong, K. Klimovica, O. Daugulis, J. Am. Chem. Soc. 2013, 135, 9342.

[27] K. B. McMurtrey, J. M. Racowski, M. S. Sanford, Org. Lett. 2012, 14, 4094.

[28] M.-G. Braun, A. G. Doyle, J. Am. Chem. Soc. 2013, 135, 12990.

[29] S. Takemoto, V. V. Grushin, J. Am. Chem. Soc. 2013, 135, 16837.

[30] A. R. Mazzotti, M. G. Campbell, P. Tang, J. M. Murphy, T. Ritter, J. Am. Chem. Soc. 2013, 135, 14012 . 
[31] Z. Feng, Q.-Q. Min, Y.-L. Xiao, B. Zhang, X. Zhang, Angew. Chem. Int. Ed. 2014, 53, 1669.

[32] a) G. B. Hammond, J. Fluorine Chem. 2006, 127, 476; b) B. Xu, M. Mae, J. A. Hong, Y. Li, G. B. Hammond, Synthesis 2006, 803, and references cited therein.

[33] S. Fustero, B. Fernández, P. Bello, C. del Pozo, S. Arimitsu, G. B. Hammond, Org. Lett. 2007, 9, 4251.

[34] G. Blessley, P. Holden, M. Walker, J. M. Brown, V. Gouverneur, Org. Lett. 2012, 14, 2754.

[35] A. Hazari, V. Gouverneur, J. M. Brown, Angew. Chem. Int. Ed. 2009, 48, 1296.

[36] J. L. Aceña, S. Fustero, H. Liu, C. Del Pozo, M. Sánchez-Roselló, V. A. Soloshonok, A. E. Sorochinsky, J. Wang, Chem. Rev. 2014, 114, 2432.

[37] N. Shibata, K. Fukushi, T. Furukawa, S. Suzuki, E. Tokunaga, D. Cahard, Org. Lett. 2012, 14, 5366.

[38] a) D. O'Hagan, Chem. Soc. Rev. 2008, 37, 308; b) C. S. Burgey, K. A. Robinson, T. A. Lyle, P. E. J. Sanderson, S. Dale Lewis, B. J. Lucas, J. A. Krueger, R. Singh, C. Miller-Stein, R. B. White, B. Wong, E. A. Lyle, P. D. Williams, C. A. Coburn, B. D. Dorsey, J. C. Barrow, M. T. Stranieri, M. A. Holahan, G. R. Sitko, J. J. Cook, D. R. McMasters, C. M. McDonough, W. M Sanders, A. A. Wallace, F. C. Clayton, D. Bohn, Y. M. Leonard, T. J. Jr. Detwiler, J. J. Jr. Lynch, Y. Yan, Z. Chen, L. Kuo, S. J. Gardell, J. A. Shafer, J. P. Vacca, J. Med. Chem. 2003, 46, 461.

[39] Q.-Q. Min, Z. Yin, Z. Feng, W.-H. Guo, X. Zhang, J. Am. Chem. Soc. 2014, 136, 1230.

[40] For selected reviews on the use of silver in organic synthesis, see: a) J.-M. Weibel, A. Blanc, P. Pale, Chem. Rev. 2008, 108, 3149; b) M. Alvarez-Corral, M. Muñoz Dorado, I. Rodríguez-García, Chem. Rev. 2008, 108 , 3174 ; c) Y. Yamamoto, Chem. Rev. 2008, 108 3199; d) M. Naodovic, H. Yamamoto, Chem. Rev. 2008, 108, 3132; e) H. Michael, 'Silver in Organic Chemistry', Wiley: Hoboken, NJ, 2010, and references therein.

[41] a) T. Hiyama, 'Organofluorine Compounds: Chemistry and Applications', Springer Verlag, 2000; b) P. Kirsch, 'Modern Fluoroorganic Chemistry', Wiley-VCH, 2006; c) I. Ojima, T. Taguchi, 'Fluorine in medicinal chemistry and chemical biology', Wiley Online Library, 2009; d) R. D. Chambers, 'Fluorine in Organic Chemistry', Oxford, New York, 2004.

[42] a) T. Furuya, A. E. Strom, T. Ritter, J. Am. Chem. Soc. 2009, 131, 1662; b) T. Furuya, T. Ritter, Org. Lett. 2009, 11, 2860; c) P. Tang, T. Furuya, T. Ritter, J. Am. Chem. Soc. 2010, 132, 12150.

[43] For the silver-mediated fluorination of vinyl stannanes, see: a) M. A. Tius, J. K. Kawakami, Synth. Commun. 1992, 22, 1461; b) M. A. Tius, J. K. Kawakami, Synlett 1993, 207; c) M. A. Tius, J. K. Kawakami, Tetrahedron 1995, 51, 3997.

[44] N. A. Petasis, A. K. Yudin, I. A. Zavialov, G. K. S. Prakash, G. A. Olah, Synlett 1997, 606.

[45] Y. Hamashima, M. Sodeoka, Synlett 2006, 1467.

[46] F. Yin, Z. Wang, Z. Li, C. Li, J. Am. Chem. Soc. 2012, 134, 10401.

[47] M. Rueda-Becerril, C. C. Sazepin, J. C. T. Leung, T. Okbinoglu, P. Kennepohl, J-F. Paquin, G. M. Sammis, J. Am. Chem. Soc. 2012, 134, 4026.

[48] C. Lai, T. E. Mallouk, J. Chem. Soc. Chem. Commun. 1993, 1359.

[49] Y. Ye, S. H. Lee, M. S. Sanford, Org. Lett. 2011, 13,5464 .

[50] a) A. Hafner, S. Bräse, Angew. Chem. Int. Ed. 2012, 51, 3713; b) A. Hafner, A. Bihlmeier, M. Nieger, W. Klopper, S. Bräse, J. Org. Chem. 2013, 78, 7938.

[51] X. Wang, Y. Xu, F. Mo, G. Ji, D. Qiu, J. Feng, Y.
Ye, S. Zhang, Y. Zhang, J. Wang, J. Am. Chem. Soc. 2013, 135, 10330.

[52] G. Liu, Org. Biomol. Chem. 2012, 10, 6243.

[53] a) T. Xu, X. Mu, H. Peng, G. Liu, Angew. Chem. Int. Ed. 2011, 50, 8176; b) T. Xu, G. Liu, Org. Lett. 2012 14, 5416; c) Q. Liu, Y. Wu, P. Chen, G. Liu, Org. Lett. 2013, 15, 6210.

[54] L. Yang, Y. Ma, F. Song, J. You, Chem. Commun. 2014, 50, 3024.

[55] H. Junbin, X. Bo, G. B. Hammond, Org. Lett. 2011, 13, 3450

[56] C.-P. Zhang, Z.-L. Wang, Q.-Y. Chen, C.-T. Zhang, Y.-C. Gu, J.-C. Xiao, Chem. Commun. 2011, 47, 6632

[57] A. Deb, S. Manna, A. Modak, T. Patra, S. Maity, D. Maiti, Angew. Chem. Int. Ed. 2013, 52, 9747.

[58] Z. Li, L. Song, C. Li, J. Am. Chem. Soc. 2013, $135,4640$.

[59] C. Zhang, Z. Li, L. Zhu, L. Yu, Z. Wang, C. Li, J. Am. Chem. Soc. 2013, 135, 14082.

[60] B. Gao, Y. Zhao, C. Ni, J. Hu, Org. Lett. 2014 , $16,102$.

[61] J. H. Hansen, H. M. L. Davies, Chem. Sci. 2011, 2, 457.

[62] C. Qin, H. M. L. Davies, Org. Lett. 2013, 15, 6152.

[63] M. Santelli, Tetrahedron 2003, 59, 701.

[64] Y. Zeng, L. Zhang, Y. Zhao, C. Ni, J. Zhao, J. Hu, J. Am. Chem. Soc. 2013, 135, 2955.

[65] K.-P. Wang, S. Y. Yun, P. Mamidipalli, D. Lee, Chem. Sci. 2013, 4, 3205.

[66] S. Y. Yun, K.-P. Wang, M. Kim, D. Lee, J. Am. Chem. Soc. 2010, 132, 8840.

[67] For the pioneering work on the preparation of fluoroalkyl ethers and thioethers, see: a) I. G. Farbenind, Patent D.R.P. 682,971, 1936. Blst. 1965, 6, III, 1009, 1033, 1034, 1038, 1067; b) F. Muller, O. Scherer, W. Schumacher, Patent US 2,108,606, 1938. Chem. Abstr. 1938, 32, 2958; c) I. G. Farbenindustrie, Fr. Patent 820,796, 1937. Zbl. 1938, 1, 1876; d) W. A. Gregory, U.S. Patent 2,763,692, 1956. Chem. Abstr. 1957, 51, 4429; e) L. M. Yagupolskii, M. S. Marenets, Zh. Obshch. Khim. 1954, 24, 887; f) L. M. Yagupolskii, Dokl. Akad. Nauk SSSR 1955, 105 , 100; g) J. F. Harris, J. Am. Chem. Soc. 1962, 84, 3148; h) J. F. Harris, F. W. Stacey, J. Am. Chem. Soc. 1961, 83, 840

[68] a) F. R. Leroux, B. Manteau, J.-P. Vors, S. Pazenok, Beilstein J. Org. Chem. 2008, 4, 13. b) F. R. Leroux, P. Jeschke, M. Schlosser, Chem. Rev. 2005, 105, 827; c) A. Tlili, T. Billard, Angew. Chem. Int. Ed. 2013, 52, 6818; d) F. Toulgoat, S. Alazet, T. Billard, Eur. J. Org. Chem. 2014, DOI: 10.1002/ejoc.201301857 early view.

[69] C. Huang, T. Liang, S. Harada, E. Lee, T. Ritter, J. Am. Chem. Soc. 2011, 133, 13308.

[70] A. Kolomeitsev, M. Vorobyev, H. Gillandt, Tetrahedron Lett. 2008, 49, 449.

[71] H.J. Emeleus, D.E. MacDuffie, J. Chem. Soc. 1961, 2597.

[72] a) L.M. Yagupolskii, N. V. Kondratenko, V. P. Sambur, Synthesis 1975, 721; b) J. H. Clark, C. W. Jones, A. P. Kybett, J. Fluorine Chem. 1990, 48, 249.

[73] F. Yin, X.-S. Wang, Org. Lett. 2014, 16, 1128.

[74] V. C. R. McLoughlin, J. Thrower, Tetrahedron 1969, 25, 5921.

[75] a) Y. Kobayashi, I. Kumadaki, Tetrahedron Lett. 1969, 10, 4095; b) Y. Kobayashi, K. Yamamoto, I. Kumadaki, Tetrahedron Lett. 1979, 20, 4071.

[76] a) G. E. Carr, R. D. Chambers, T. F. Holmes, D. G. Parker, J. Chem. Soc. Perkin Trans. 1 1988, 921; b) D.-B. Su, J.-X. Duan, Q.Y. Chen, Tetrahedron Lett. 1991, 32, 7689; c) Q.-Y. Chen, J.-X. Duan, J. Chem. Soc. Chem. Commun. 1993, 1389; d) H. Urata, T. Fuchikami, Tetrahedron Lett. 1991, 32, 91; e) A. Kutt, V. Movchun, T. Rodima, T. Dansauer, E. B. Rusanov, I. Leito, I. Kaljurand, J. Koppel, V. Pihl, I. Koppel, G. Ovsjannikov, L. Toom,
M. Mishima, M. Medebielle, E. Lork, G.-V. Roschenthaler, I. A. Koppel, A. A. Kolomeitsev, J. Org. Chem. 2008, 73, 2607; f) F. Cottet, M. Schlosser, Eur. J. Org. Chem. 2002, 327; g) F. Cottet, M. Schlosser, Eur. J. Org. Chem. 2003, 1559; h) G. G. Dubinina, H. Furutachi, D. A. Vicic, J. Am. Chem. Soc. 2008, 130, 8600; i) G. G. Dubinina, J. Ogikubo, D. A. Vicic, Organometallics 2008, 27, 6233 .

[77] Q. Chen, S. Wu, J. Chem. Soc. Chem. Commun. 1989, 705.

[78] M. Oishi, H. Kondo, H. Amii, Chem. Commun. 2009, 1909.

[79] H. Morimoto, T. Tsubogo, N. D. Litvinas, J. F. Hartwig, Angew. Chem. Int. Ed. 2011, 50, 3793; b) N. D. Litvinas, P. S. Fier, J. F. Hartwig, Angew. Chem. Int. Ed. 2012, 51, 536.

[80] For selected examples of trifluoromethylation of arylboron derivatives, see: a) T. Liu, Q. Shen, Org. Lett. 2011, 13, 2342; b) C.-P. Zhang, J. Cai, C.-B. Zhou, X.-P. Wang, X. Zheng, Y.-C. Gu, J.C. Xiao, Chem. Commun. 2011, 47, 9516; c) J. Xu, D.-F. Luo, B. Xiao, Z.-J. Liu, T.-J. Gong, Y. Fu, L. Liu, Chem. Commun. 2011, 47, 4300; d) L. Chu, F.-L. Qing, Org. Lett. 2010, 12, 5060; e) X. Jiang, L. Chu, F.-L. Qing, J. Org. Chem. 2012, 77, 1251; f) T. D. Senecal, A. T. Parsons, S. L. Buchwald, J. Org. Chem. 2011, 76, 1174; g) B. A. Khan, A. E. Buba, L. J. Gooßen, Chem. Eur. J. 2012, 18, 1577.

[81] M. Chen, S. L. Buchwald, Angew. Chem. Int. Ed. 2013, 52, 11628.

[82] F. Cottet, M. Schlosser, Eur. J. Org. Chem. $2002,327$.

[83] G. G. Dubinina, H. Furutachi, D. A. Vicic, J. Am. Chem. Soc. 2008, 130, 8600.

[84] J. Dai, C. Fang, B. Xiao, J. Yi, J. Xu, Z. Liu, X. Lu, L. Liu, Y. Fu, J. Am. Chem. Soc. 2013, 135, 8436.

[85] G. Danoun, B. Bayarmagnai, M. F. Grünberg, L. J. Gooßen, Angew. Chem. Int. Ed. 2013, 52, 7972.

[86] W. Han, Y. Li, H. Tang, H. Liu, J. Fluorine Chem. 2012, 140, 7

[87] a) A. Zanardi, M. Novikov, E. Martin, J. BenetBuchholz, V. V. Grushin, J. Am. Chem. Soc. 2011, 133, 20901; b) P. Novak, A. Lishchynskyi, V. V. Grushin, Angew. Chem. Int. Ed. 2012, 51, 7767; c) P. Novak, A. Lishchynskyi, V. V. Grushin, J. Am. Chem. Soc. 2012, 134, 16167; d) A. Lishchynskyi, M. Novikov, E. Martin, E. C. Escudero-Adán, P. Novák, V. V. Grushin, J. Org. Chem. 2013, 78, 11126.

[88] G. K. S. Prakash, P. V. Jog, P. T. D. Batamack, G. A. Olah, Science 2012, 338, 1324.

[89] a) J. S. Houlton,W. B. Motherwell, B. C. Ross, M. J. Tozer, D. J. Williams, A. M. Z. Slawin, Tetrahedron 1993, 49, 8087; b) S. Kaneko, T. Yamazaki, T. Kitazume, J. Org. Chem. 1993, 58, 2302.

[90] W. F. Goure, K. L. Leschinsky, S. J. Wratten, J. P. Chupp, J. Agric. Food Chem. 1991, 39, 981.

[91] K. Fujikawa, Y. Fujioka, A. Kobayashi, H. Amii, Org. Lett. 2011, 13, 5560.

[92] P. S. Fier, J. F. Hartwig, J. Am. Chem. Soc. 2012, $134,5524$.

[93] G. K. S. Prakash, S. K. Ganesh, J.-P. Jones, A. Kulkarni, K. Masood, J. K. Swabeck, G. A. Olah, Angew. Chem. Int. Ed. 2012, 51, 12090.

[94] F. Wang, T. Luo, J. Hu, Y. Wang, H. S. Krishnan, P. V. Jog, S. K. Ganesh, G. K. S. Prakash, G. A. Olah, Angew. Chem. Int. Ed. 2011, 50, 7153.

[95] A. Casitas, M. Canta, M. Solà, M. Costas, X. Ribas, J. Am. Chem. Soc. 2011, 133, 19386.

[96] Y. Ye, M. S. Sanford, J. Am. Chem. Soc. 2013, $135,4648$.

[97] Y. Ye, S. D. Schimler, P. S. Hanley, M. S. Sanford, J. Am. Chem. Soc. 2013, 135, 16292.

[98] N. O. Ilchenko, P. G. Janson, K. J. Szabó, Chem. Commun. 2013, 49, 6614

[99] X. Wang, Y. Ye, G. Ji, Y. Xu, S. Zhang, J. Feng, Y. Zhang, J. Wang, Org. Lett. 2013, 15, 3730.

[100] C. Feng, T.-P. Loh, Chem. Sci. 2012, 3, 3458. 
[101] C. Feng, T.-P. Loh, Angew. Chem. Int. Ed. 2013, 52, 12414.

[102] T. Besset, D. Cahard, X. Pannecoucke, J. Org. Chem. 2014, 79, 413

[103] Z. Fang, Y. Ning, P. Mi, P. Liao, X. Bi, Org. Lett. 2014, 16, 1522.

[104] Z. Mao, F. Huang, H. Yu, J. Chen, Z. Yu, Z. $\mathrm{Xu}$, Chem. Eur. J. 2014, 20, 3439.

[105] P. G. Janson, I. Ghoneim, N. O. Ilchenko, K. J Szabó, Org. Lett. 2012, 14, 2882.

[106] H. Egami, R. Shimizu, M. Sodeoka, Tetrahedron Lett. 2012, 53, 5503.

[107] R. Zhu, S. L. Buchwald, J. Am. Chem. Soc. 2012, 134, 12462.

[108] X.-Y. Jiang, F.-L. Qing, Angew. Chem. Int. Ed. 2013, 52, 14177

[109] F. Wang, X. Qi, Z. Liang, P. Chen, G. Liu, Angew. Chem. Int. Ed. 2014, 53, 1881.

[110] G. Teverovskiy, D. S. Surry, S. L. Buchwald, Angew. Chem. Int. Ed. 2011, 50, 7312.

[111] C. Chen, Y. Xie, L. Chu, R.-W. Wang, X Zhang, F.-L. Qing, Angew. Chem. Int. Ed. 2012, 51, 2492.

[112] W. Tyrra, D. Naumann, B. Hoge, Y. L. Yagupolskii, J. Fluorine Chem. 2003, 119, 101.

[113] Z. Weng, W. He, C. Chen, R. Lee, D. Tan, Z. Lai, D. Kong, Y. Yuan, K.-W. Huang, Angew. Chem. Int. Ed. 2013, 52, 1548.

[114] D. Kong, Z. Jiang, S. Xin, Z. Bai, Y. Yuan, Z. Weng, Tetrahedron 2013, 69, 6046.

[115] Z. Wang, Q. Tu, Z. Weng, J. Organomet. Chem. 2014, 751, 830 .

[116] M. Rueping, N. Tolstoluzhsky, P. Nikolaienko, Chemistry 2013, 19, 14043.

[117] R. Pluta, P. Nikolaienko, M. Rueping, Angew. Chem. Int. Ed. 2014, 53, 1650.

[118] Reviews on benziodoxole and benziodoxolone reagents: a) V. V. Zhdankin, Curr. Org. Synth. 2005, 2, 121; b) J. P. Brand, D. F. Gonzalez, S. Nicolai, J. Waser, Chem. Commun. 2011, 47, 102.

[119] For hypervalent iodine $\mathrm{CF}_{3} \mathrm{~S}$ group transfer reagents, see: a) P. Eisenberger, S. Gischig, A. Togni, Chem. Eur. J. 2006, 12, 2579; b) I. Kieltsch, P. Eisenberg, K. Stanek, A. Togni, Chimia 2008, 62, 260; c) K. Niedermann, N. Fruh, E. Vinogradova, M. S.Wiehn, A. Moreno, A. Togni, Angew. Chem. Int. Ed. 2011, 50, 1059; d) Q.-H. Deng, H. Wadepohl, L. H. Gade, J. Am. Chem. Soc. 2012, 134 , 10769; e) X. Liu, X. Wu, Synlett 2013, 1882.

[120] X. Shao, X. Wang, T. Yang, L. Lu, Q. Shen, Angew. Chem. 2013, 125, 3541; Angew. Chem. Int. Ed. 2013, 52, 3457.

[121] E. V. Vinogradova, P. Müller, S. L. Buchwald, Angew. Chem. Int. Ed. 2014, 53, 3125.

[122] Y. Inokuma, S. Yoshioka, J. Ariyoshi, T. Arai, Y. Hitora, K. Takada, S. Matsunaga, K Rissanen, M. Fujita, Nature 2013, 495, 461.

[123] a) G. C. Vougioukalakis, R. H. Grubbs, Chem. Rev. 2010, 110, 1746; b) A. M. Lozano-Vila, S. Monsaert, A. Bajek, F. Verpoort, Chem. Rev. 2010, 110, 4865; c) C. Samoj?owicz, M. Bieniek, K. Grela, Chem. Rev. 2009, 109, 3708.

[124] G. Magueur, J. Legros, F. Meyer, M. Ourévitch, B. Crousse, D. Bonnet-Delpon, Eur. J. Org. Chem. 2005, 1258.

[125] F. Grellepois, V. Kikelj, N. Coia, C. Portella, Eur. J. Org. Chem. 2012, 509.

[126] a) M. Eckert, F. Monnier, G. T. Shchetnikov, I. D. Titanyuk, S. N. Osipov, L. Toupet, S. Dérien, P. H. Dixneuf, Org. Lett. 2005, 7 , 3741; b) M. Eckert, S. Moulin, F. Monnier, I. D. Titanyuk, S. N. Osipov, T. Roisnel, S. Dérien, P. H. Dixneuf, Chem. Eur. J. 2011 17, 9456; c) A. K. Mailyan, I. M. Krylov, C. Bruneau, P. H. Dixneuf, S. N. Osipov, Eur. J. Org. Chem. 2013, 5353.

[127] For selected examples, see: a) G. C. LloydJones, R. G. Margue, J. G. de Vries, Angew. Chem. Int. Ed. 2005, 44, 7442; b) A. Kinoshita, N. Sakakibara, M. Mori, Tetrahedron 1999, 55,8155 ; c) A. Kinoshita, N. Sakakibara, M. Mori, J. Am. Chem. Soc. 1997, 119, 12388.
[128] a) S. A. Pujari, K. P. Kaliappan, A. Valleix, D. Grée, R. Grée, Synlett 2008, 2503; b) S. Fustero, P. Bello, J. Miró, A. Simón, C. del Pozo, Chem. Eur. J. 2012, 18, 10991; c) S. Fustero, P. Bello, J. Miró, M. SánchezRoselló, G. Haufe, C. del Pozo, Beilstein J. Org. Chem. 2013, 9, 2688.

[129] a) S. Fustero, D. Jiménez, M. SánchezRoselló, C. del Pozo, J. Am. Chem. Soc. 2007, 129, 6700; b) S. Fustero, S. Monteagudo, M. Sánchez-Roselló, S. Flores, P. Barrio, C. del Pozo, Chem. Eur. J. 2010, 16, 9835.

[130] S. Fustero, C. Báez, M. Sánchez-Roselló, A. Asensio, J. Miró, C. del Pozo, Synthesis 2012, 44, 1863.

[131] S. V. Slungãrd, T.-A. Krakeli, T. H. K. Thvedt, E. Fuglseth, E. Sundby, B. H. Hoff, Tetrahedron 2011, 67, 5642.

[132] T. Otsuka, A. Ishii, P. A. Dub, T. Ikariya, $J$. Am. Chem. Soc. 2013, 135, 9600.

[133] B. Sam, T. P. Montgomery, M. J. Krische, Org. Lett. 2013, 15, 3790.

[134] a) C. Müller, R. J. Lachicotte, W. D. Jones, Organometallics 2002, 21, 1975; b) T. Konno, K. Moriyasu, R. Kinugawa, T. Ishihara, Org. Biomol. Chem. 2010, 8, 1718.

[135] A. T. Herrmann, L. L. Smith, A. Zakarian, J. Am. Chem. Soc. 2012, 134, 6976.

[136] a) S. Beaumont, E. A. Ilardi, L. R. Monroe, A. Zakarian, J. Am. Chem. Soc. 2010, 132, 1482; b) Z. Gu, A. T. Herrmann, A. Zakarian, Angew. Chem. Int. Ed. 2011, 50, 7136; c) T. Amatov, U. Jahn, Angew. Chem. Int. Ed. 2011, 50,4542 .

[137] a) S. Mizuta, K. M. Engle, S. Verhoog, O. Galicia-López, M. O’Duill, M. Médebielle, K. Wheelhouse, G. Rassias, A. L. Thompson, V. Gouverneur, Org. Lett. 2013, 15, 1250; b) S. Mizuta, S. Verhoog, K. M. Engle, T. Khotavivattana, M. O'Duill, K. Wheelhouse, G. Rassias, M. Médebielle, V. Gouverneur, J. Am. Chem. Soc. 2013, 135, 2505. 